\title{
Multiscale Modeling of Protein Adsorption in Polymer-Grafted Ion Exchangers
}

\author{
A Dissertation \\ Presented to \\ the faculty of the School of Engineering and Applied Science \\ University of Virginia \\ in partial fulfillment \\ of the requirements for the degree \\ Doctor of Philosophy \\ by \\ Joseph E. Basconi
}

May 2015 
APPROVAL SHEET

The dissertation

is submitted in partial fulfillment of the requirements

for the degree of

Doctor of Philosophy

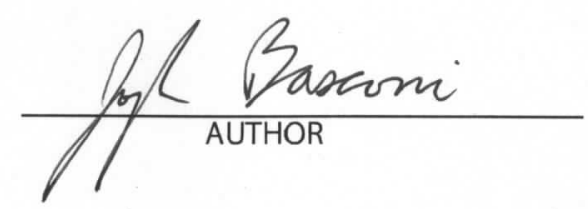

The dissertation has been read and approved by the examining committee:

Michael R. Shirts

\begin{tabular}{c}
\hline $\begin{array}{c}\text { Advisor } \\
\text { Giorgio Carta }\end{array}$ \\
\hline Roseanne M. Ford \\
\hline David S. Cafiso \\
\hline Leonid V. Zhigilei \\
\hline
\end{tabular}

Accepted for the School of Engineering and Applied Science:

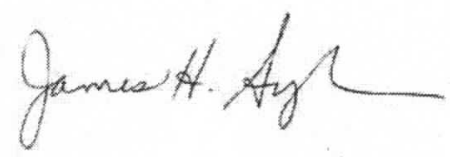

Dean, School of Engineering and Applied Science

May

2015 


\section{Abstract}

This work examines how molecular properties affect protein adsorption in polymergrafted ion exchange chromatography (IEC) resins, as predicted by multiscale computational modeling. Polymer-grafted IEC resins, which have charged polymers grafted into their pores, are widely used because they can enhance the protein binding capacity and adsorption kinetics relative to traditional macroporous resins with open pore structures. Multiscale modeling is used to elucidate the molecular details of protein adsorption and diffusion in a polymer-grafted pore and to predict how these molecular behaviors affect experimentally-relevant macroscopic adsorption properties.

Our multiscale modeling approach combines molecular dynamics (MD) simulation of protein in an IEC pore with numerical simulation of mass transfer into a resin particle. The molecular models are designed based on both the known physical properties of the systems of interest and on the experimentally-observed adsorption behaviors for these systems. Initial simulations of lysozyme in both macroporous and polymer-grafted resin pores agree qualitatively with experiments, showing that the polymer grafts have a modest effect on the adsorption capacity relative to the macroporous resin, but can enhance the effective transport rate significantly when electrostatic interactions are strong. This behavior arises from the combination of enhanced protein partitioning into the polymer-filled 
pore space, and relatively fast diffusion of protein associated with the polymers. Additional studies predict that lysozyme's adsorption capacity and kinetics can be enhanced by increasing either the resin's polymer graft density or the per-polymer charge content, as both types of modifications increase the number of polymer ligands available for adsorption within the pore. Systems with higher polymer ligand contents also exhibit more diffuse adsorption fronts. In systems with a high charge content per polymer and a low protein loading, the polymers preferentially partition towards the surface due to favorable interactions with the surface-bound protein.

Simulations of lysozyme with different net charges, BSA, and IgG1 predict that adsorption behaviors vary significantly with the properties of the protein. For the polymer-grafted system, protein partitioning into the pore space and the overall transport rate are predicted to increase with the charge of the protein. Analysis of the number of contacts made between protein molecules and polymer ligands and protein mobility in the polymer-filled pore support existing hypotheses on the chain delivery mechanism for diffusion in these systems. 


\section{Acknowledgments}

I am grateful for the support of various mentors, fellow students, friends, and family in helping me complete this work. Thanks to my first research advisor, Professor Jack Hudson, whose wisdom and perspective will not be forgotten. Thanks as well to Prof. Giorgio Carta, for introducing me to the field of protein chromatography and providing me the opportunity to do both modeling and laboratory research. Thanks to Professor Michael Shirts, for guiding my modeling work and helping me improve my research, writing, and presentation skills. I'd also like to thank the members of my Ph.D. committee, Professors Ford, Cafiso, and Zhigeli, for their careful review of my work. Thanks as well to Vickie, Teresa, Jennifer, and Kim for their help over the years.

I very much appreciate the financial support I have received to do this research. Thanks to the U.S. National Science Foundation for their grant, and the UVa Chemical Engineering Department for providing additional funding. Thanks as well to UVa Advanced Computing Services and Engagement and the National Institute for Computational Sciences for providing the computing resources for our simulations.

The advice and friendship of my fellow students at UVa have been invaluable in completing this work. In particular, thanks to Jing Guo for helping me learn experimental techniques, and to Ed Wong for countless helpful discussions. 
Thanks as well to Simpson, Juan, Tarl, Jacob, Ernie, Yige, Mimi, Shaoije, Drew, Arch, Jason, Himanshu, Kai, Levi, Brittany, Eric, Karen, Matthew, Craig, Xuan, and the many Shirts group undergraduates for your help and friendship.

I'd like to thank my friends in Charlottesville from various circles for making this a wonderful town in which to study. I owe big thank yous to my parents, sisters, and extended family for their unfailing support. Their granola shipments, paper proofreads, reminders to get back to work, and prayers were much appreciated. Finally, thanks to God for creating proteins and minds to study them. 


\section{Contents}

1 Motivation and Background $\quad 1$

1.1 Background .......................... 1

1.1.1 Downstream Processing and IEC . . . . . . . . . . . 1

1.1.2 Macroporous and IEC Resins . . . . . . . . . . . . 2

1.1.3 Mass Transfer Mechanisms . . . . . . . . . . . . . . . 4

1.1.4 Experimental Results . . . . . . . . . . . . 5

1.1.5 The Basis for Mass Transfer in Polymer-grafted Resins . . . . 6

1.1.6 Opportunities for Study by Molecular Modeling . . . . . . 9

1.2 Scope and Outline of this Dissertation . . . . . . . . . . 9

2 Model Development $\quad 11$

2.1 Introduction ......................... 11

2.1.1 Background ....................... 12

2.2 Development of molecular models . . . . . . . . . . . . . . . 14

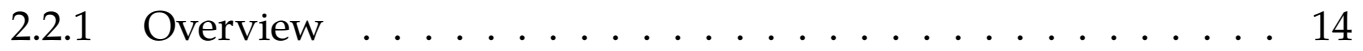

2.2.2 Justification of coarse-grained models . . . . . . . . . . . 16

2.2.3 Comparison with Atomistic Simulation . . . . . . . . . . 17

2.2.4 Parameterizing the CG Models . . . . . . . . . . . . . . . . 20

2.2.5 Model Details . . . . . . . . . . . . . . . 21 
2.3 Data Analysis Methods . . . . . . . . . . . . . . . . . 28

2.3.1 Adsorption isotherms . . . . . . . . . . . . . 28

2.3.2 Molecular diffusivities . . . . . . . . . . . . . . . . . 31

2.3.3 multiscale simulation of adsorption kinetics . . . . . . . 32

2.4 Model parametrization . . . . . . . . . . . . . . . . . . 34

2.4.1 Effects of protein diffusion and partitioning on macroscopic adsorption kinetics . . . . . . . . . . . . . . . . . 34

2.4.2 Effects of molecular model parameters on protein diffusion and partitioning $\ldots \ldots \ldots \ldots \ldots \ldots$

2.5 Effects of strength of electrostatics on adsorption behavior . . . . . 42

2.5.1 Adsorption Equilibria . . . . . . . . . . . . . . . . 42

2.6 Molecular diffusivities . . . . . . . . . . . . . . . . . . 47

2.6 .1 Adsorption kinetics . . . . . . . . . . . . . 50

2.7 Discussion . . . . . . . . . . . . . . . . . 54

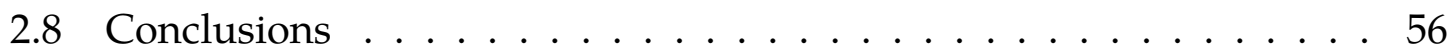

3 Effects of Polymer Graft Properties $\quad 58$

3.1 Introduction $\ldots \ldots \ldots \ldots \ldots \ldots \ldots \ldots \ldots \ldots \ldots \ldots \ldots \ldots \ldots$

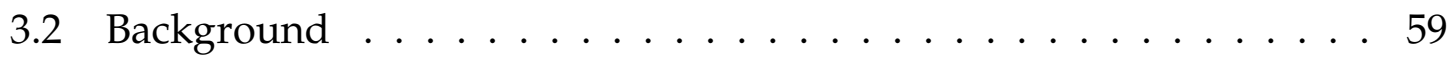

3.3 Molecular simulation details $\ldots \ldots \ldots \ldots$. . . . . . . 61

3.4 Results . . . . . . . . . . . . . . . . 62

3.4.1 Adsorption Equilibria _ . . . . . . . . . . . . . 62

3.4.2 Molecular diffusivities . . . . . . . . . . . . . . 68

3.4 .3 Adsorption kinetics . . . . . . . . . . . . 70

3.4.4 Polymer phase properties . . . . . . . . . . . . . . 74

3.5 Conclusions . . . . . . . . . . . . . . . . . . 79 
4 Effects of Protein Properties $\quad 81$

4.1 Introduction . . . . . . . . . . . . . . 81

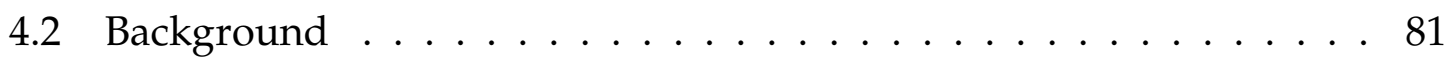

4.3 Methods ............................. 84

4.3.1 MD simulation details . . . . . . . . . . . . . . . 84

4.4 Results ........................ 89

4.4 .1 Adsorption Equilibria ................. 89

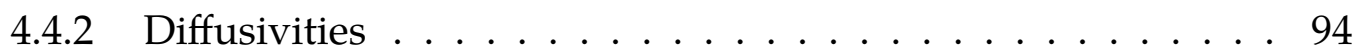

4.4 .3 Adsorption kinetics ..................... 98

4.4.4 Protein-polymer interactions ... . . . . . . . . 103

4.4 .5 Conclusions . . . . . . . . . . . . . . . 109

5 Conclusions and Recommendations $\quad 110$

5.1 Conclusions . . . . . . . . . . . . . . . . 110

5.2 Recommendations ........................ 111

$\begin{array}{ll}\text { A Molecular model details } & 114\end{array}$

A.1 Protein model . . . . . . . . . . . . . . . . . . . . . 114

A.2 Polymer graft model . . . . . . . . . . . . . . . . 118

A.2.1 Solvent Environment . . . . . . . . . . . . . . . 120

$\begin{array}{lll}\text { B Model parameterization simulations } & 124\end{array}$

$\begin{array}{ll}\text { References } & 126\end{array}$ 


\section{List of Figures}

1.1 Illustration of an open macropore and a polymer-grafted IEC resin

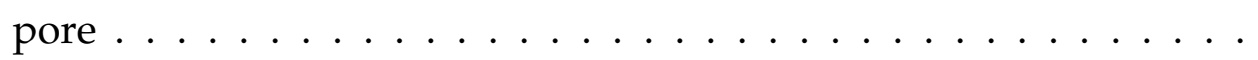

2.1 Snapshots from MD simulations of lysozyme within an open macropore and a polymer-grafted pore . . . . . . . . . . . . 15

2.2 Snapshots from MD simulation of an atomistic sulfonated dextran

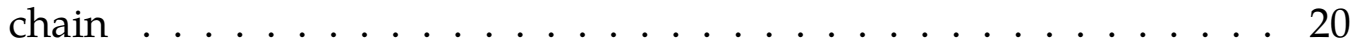

2.3 Mapping of lysozyme $\alpha$-carbon atoms to CG model . . . . . . . 23

2.4 Snapshot from MD simulation of lysozyme in polymer-grafted system with void region adjacent to pore . . . . . . . . . . 27

2.5 Histograms of average distance between lysozyme center of mass and nearest surface ligand . . . . . . . . . . . . 30

2.6 Predicted $D_{e, a p p} / D_{0}$ values for lysozyme in a macroporous system as a function of various simulation parameters $\ldots . \ldots 36$

2.7 Predicted $D_{e, a p p} / D_{0}$ values for lysozyme in a polymer-grafted system as a function of various simulation parameters . . . . . . . 38

2.8 Adsorption isotherms for lysozyme in macroporous and polymergrafted systems, as determined by MD simulation . . . . . . . . . 43 
2.9 MSD curves and corresponding diffusivities for lysozyme in macroporous and polymer-grafted systems, as determined by MD simu-

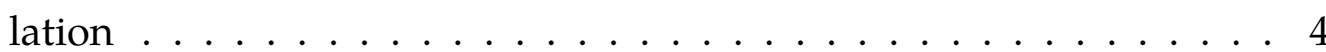

2.10 Intraparticle concentration profiles and batch uptake curves for lysozyme in different adsorbents, as predited by multiscale sim-

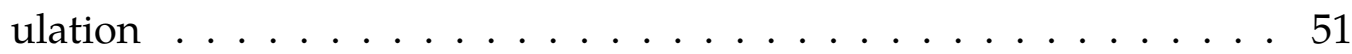

2.11 Comparison of $D_{e, \text { app }} / D_{0}$ values of lysozyme in different adsorbents determined by multiscale simulation and from previous experiments 53

3.1 Snapshots from MD simulations of lysozyme in polymer-grafted pores with different polymer graft densities . . . . . . . . . 61

3.2 Adsorption isotherms for lysozyme in IEC pores with different polymer graft properties, as predicted by MD simulation. . . . . . 65

3.3 Saturation capacities for lysozyme in IEC pores with different polymer graft properties, as predicted by MD simulation. . . . . . . . 67

3.4 Pore space saturation capacities for lysozyme in IEC pores with different polymer graft properties, as predicted by MD simulation. 68

3.5 Pore space diffusivities for lysozyme in IEC pores with different polymer graft properties, as predicted by MD simulation. . . . . . 70

3.6 $D_{e, a p p} / D_{0}$ values for lysozyme in IEC pores with different polymer graft properties, as predicted by multiscale simulation. . . . . . . 72

3.7 Intraparticle concentration profiles for lysozyme in IEC pores with different polymer graft properties, as predicted by multiscale simulation. . . . . . . . . . . . . . . . . . . . . . . 74

3.8 Number density of lysozyme within different polymer-grafted pores under relatively low and high protein loadings, as predicted by multiscale simulation. . . . . . . . . . . . . . . . 75 
3.9 Number density of lysozyme within a polymer-grafted pore as a function of local protein concentration, as predicted by multiscale

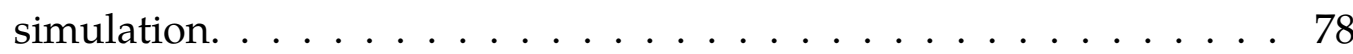

4.1 Side by side comparison of CG models of the different proteins studied in this work . . . . . . . . . . . . 85

4.2 Snapshots from MD simulations of various proteins in a polymer-

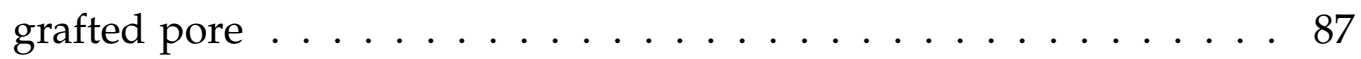

4.3 Histograms of minimum distances between protein and nearest surface ligand . . . . . . . . . . . . . . . . . . . 89

4.4 Adsorption isotherms of lysozyme charge variants in polymer-grafted and macroporous systems predicted by MD simulation . . . . . 90

4.5 Adsorption isotherms of BSA in polymer-grafted and macroporous systems predicted by MD simulation . . . . . . . . . . . 92

4.6 Adsorption isotherms of IgG1 in polymer-grafted and macroporous adsorbents, predicted by MD simulations . . . . . . . . . . . 93

4.7 Average diffusivities of lysozyme charge variants in different regions of the polymer-grafted system, as predicted by MD simula-

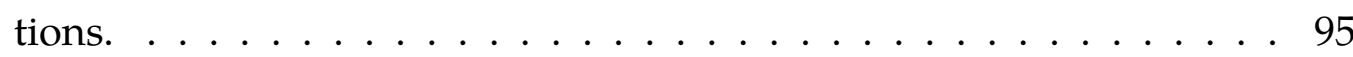

4.8 Average diffusivities of BSA and IgG1 in different regions of IEC

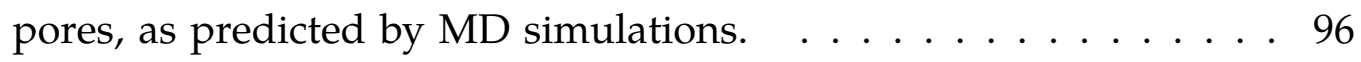

4.9 Intraparticle concentration profiles for various proteins as predicted by multiscale simulation of mass transfer . . . . . . . . . 99

$4.10 D_{e, a p p} / D_{0}$ values for various proteins in polymer-grafted and macroporous systems, as predicted by multiscale simulation of mass transfer . . . . . . . . . . . . . . . . . . . . . 101 
4.11 Histograms of the average number of polymer ligands with which the protein in the pore space is in contact . . . . . . . . . . . 104

4.12 Histograms of protein displacement over a given time window versus the average number of polymer ligand contacts during that

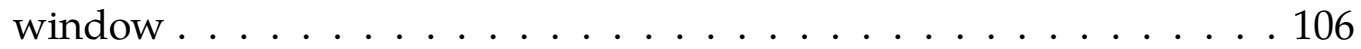

A.1 Comparison of electrostatic potential evaluated around CG and atomistic models for lysozyme . . . . . . . . . . . . 116

A.2 Standard errors of least squares fits to $V_{C G}$ vs. $V_{\text {atom }}$ for all tested CG lysozyme models. . . . . . . . . . . . . . . . . 116

A.3 Radial distribution function of lysozyme relative to surface ligands as a function of cutoff radius for reaction field electrostatic potential 123

B.1 Number density of lysozyme within an open macropore for two different surface models, as determined by MD simulation . . . . . 126 


\section{List of Tables}

2.1 Summary of model parameters used for production MD simulations and results from molecular and mass transfer simulations based on these parameters . . . . . . . . . . . . 40

2.2 Isotherm model parameters for lysozyme in macroporous and polymergrafted systems, as determined by MD simulation . . . . . . . . . 46

2.3 Diffusivity of lysozyme in the polymer-filled pore space as a function of pore space concentration, as predicted by MD simulation . . 49

4.1 Molecular properties and PDB codes of proteins studied in this work 85

B.1 Summary of lysozyme adsorption properties predicted by multiscale simulation with various input parameters . . . . . . . 125

B.2 Diffusivity of surface-bound lysozyme as a function of surface model parameters, determined by MD simulation . . . . . . . . . . . . 127 


\section{Chapter 1}

\section{Motivation and Background}

\subsection{Background}

\subsubsection{Downstream Processing and IEC}

Advances in downstream processing will be necessary for biopharmaceutical production to meet demand in coming years. The share of biological products within the overall pharmaceutical market is growing steadily from $11 \%$ in 2002 to an expected $20 \%$ in 2017. [1] The increased production that has made this growth possible has primarily been driven by advances in upstream processing. More efficient cell lines and bioreactors have increased protein titers from tens of milligrams to more than 10 grams per liter over the past 20 years. [2] Downstream processing of this product is now often the rate-limiting step in manufacturing. $[3,4]$ Improving the stationary phases, or resins, for downstream protein chromatography steps used to capture and purify the biologic of interest is one approach towards meeting these production challenges as well as the product purity requirements.

Ion exchange chromatography (IEC) is an important unit operation in the 
downstream processing of many biologics. [5] In this mode of chromatography, a charged molecule reversibly binds to an oppositely charged stationary phase. Positively charged adsorbates bind to negatively charged cation exchange resins and in the process displace bound positive counter ions from the mobile phase. Similarly, negatively charged adsorbates bind to an anion exchange resin with a positive charge and displace mobile phase anions. IEC is particularly useful for protein separations because for each protein species, there is a unique relationship between the net charge and the solution $\mathrm{pH}$, often shown in a titration curve. Protein species interact with an oppositely charged IEC resin to varying degrees based on their different net charges at a given $\mathrm{pH}$, thus allowing the product of interest to be separated from impurities such as product isoforms, aggregated product, viruses, and impurities from the host cell used in upstream production. [5]

\subsubsection{Macroporous and IEC Resins}

IEC resins consist of a base matrix that is functionalized with anion or cation substituents, which provide the means for capturing oppositely-charge molecules. The optimal base matrix and functional groups depend on the protein to be separated as well as process requirements. Natural carbohydrates are often used for the base matrix because they provide high porosities, which lead to large surface areas for functionalization and therefore high binding capacities. [6] These materials generally are fairly hydrophilic, which helps minimize non-specific interactions with non-targeted molecules. Agarose is a commonly-used polymer, which upon cooling from a hot aqueous state, forms a gel with a macroporous structure through which proteins can diffuse. The mechanical stability of the resulting particles can be improved by chemically cross-linking the agarose monomers, 
which is necessary for running at high flow rates in commercial applications. On the other hand, softer carbohydrates such as cellulose or freestanding hydrogels can offer very high binding capacities when functionalized, but at the expense of low mechanical strength. [6] Composite "polymer-grafted" resins have been developed with the aim of combining the high mechanical strength of rigid particles and the desirable adsorption properties of charged hydrogels. [7-11] These adsorbents have charged polymers grafted within the pore structure, which are thought to provide dynamic adsorption sites either in addition to or in lieu of those associated with ligands on the pore surface. The inclusion of charged polymers can allow for multi-layers of bound protein, $[10,12]$ as opposed to traditional "macroporous" resins in which typically only monolayer adsorption is possible. $[6,13,14]$ The illustration in Figure 1.1 provides a conceptual representation of the pores of a macroporous and a polymer-grafted IEC resin.

Depending on the identity and length of the polymer grafts, the structure within the particle pores can resemble a polymer brush-like layer on the surfaces with an open interior pore space, random coil structures that extend further into the pore (with single or multiple attachment points), or a hydrogel-like environment that fills the entire pore space. [15] The polymers can be based on charged monomers or electrically neutral monomers that are functionalized with charged ligands after grafting. Charged polymers have also been grafted into the pore structure of macroporous monolithic polyacrylamide cryogels [16] and microporous membranes $[12,17]$ to enhance the protein binding capacities of these materials. Additionally, various polymers have been grafted onto surfaces to reduce non-specific protein adsorption for both protein chromatography [18] and other biomedical applications. [19] 


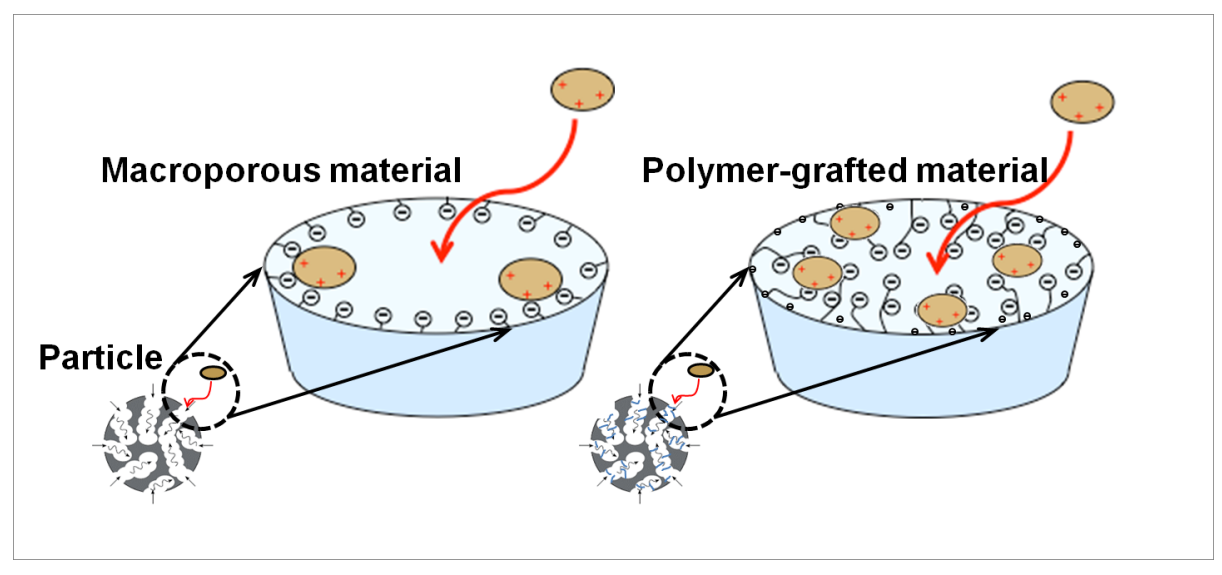

Figure 1.1: Conceptual representation of the pores of a macroporous and a polymer-grafted IEC resin.

\subsubsection{Mass Transfer Mechanisms}

Mass transfer is thought to occur by different mechanisms in macroporous and polymer-grafted IEC resins. [5] In both cases, intraparticle diffusion controls the overall transport rate. In a macroporous material, transport typically is governed by the ordinary pore diffusion mechanism with the mass transfer flux given by

$$
J=-D_{e} \nabla C
$$

where $D_{e}$ is the effective pore diffusivity and $C$ is the protein concentration in the open macropore (which is the same as the extraparticle concentration, $C_{0}$ ). As shown, the driving force for mass transfer is the concentration gradient of unadsorbed protein in the pore space. Mass transfer via ordinary pore diffusion is characterized by a sharp adsorption front. By contrast, transport in polymergrafted materials is thought to occur by the solid diffusion mechanism in which the gradient in the concentration of adsorbed protein, $q$, is the driving force for 
diffusion:

$$
J=-D^{\prime} \nabla q
$$

where $D^{\prime}$ is the effective adsorbed phase diffusivity. Generally $D^{\prime}$ is concentration dependent while $D_{e}$ is not, and typically $D^{\prime}<D_{e}$ due to the restricted nature of diffusion in the adsorbed phase. Both Eqs. 1.1 and 1.2 assume that $q$ is much larger than $C$. This type of mass transfer is typically characterized by a diffuse adsorption front.

Despite the different diffusional driving forces shown above, the two models predict adsorption kinetics that are qualitatively very similar. Thus, in studies of materials that have a solid diffusion mechanism, batch uptake curves can be fit with the pore diffusion model to obtain an apparent effective pore diffusivity, which can be compared with a true $D_{e}$ measured in a pore diffusion-controlled material. The ratio of $D_{e}$ to the protein diffusivity in the free solution, $D_{0}$, is often used to quantify the effective transport rate, as it provides a measure of the degree to which a resin enhances or hinders transport relative to diffusion in the bulk.

\subsubsection{Experimental Results}

Previous studies have demonstrated that polymer-grafted IEC resins can provide enhanced adsorption properties versus traditional macroporous resins. Stone and Carta studied the adsorption behavior of different proteins on cation exchangers consisting of porous agarose particles with either an open pore structure or with dextran polymers functionalized with sulfonate charged groups grafted to the pore surfaces. [9] The underlying agarose matrix was also functionalized with 
sulfonate groups in both materials. In single-component adsorption experiments, the binding capacities of lysozyme and an IgG1 monoclonal antibody $(\mathrm{mAb})$ were 15 and 25\% higher, respectively, in the dextran-grafted matrix than in the open pore material. $[9,20]$

Batch adsorption experiments have demonstrated that polymer-grafted resins can also enhance the adsorption kinetics. Stone and Carta quantified the effective mass transfer rate of various proteins in the resins described above by estimating the apparent effective pore diffusivity, $D_{e, a p p}$, from batch uptake curves. While low values of $D_{e, a p p}$ were obtained for the open-pore adsorbent, consistent with mass transfer via pore diffusion, surprisingly high values of $D_{e, a p p}$ that in some cases even exceeded $D_{0}$, were obtained for lysozyme and the mAb in the dextran-grafted adsorbent. Bowes and co-workers also observed enhanced adsorption capacities and kinetics in the commercial dextran-grafted materials SP Sepharose XL and Capto $S$ versus a commercial macroporous material with a similar backbone, SP Sepharose Fast Flow. [10, 21] Recent studies on the adsorption of a mAb onto commercial dextran-grafted and macroporous resins have shown that charged polymer grafts can affect the mechanism by which mass transfer occurs. [22] Confocal laser scanning microscopy (CLSM) images of intraparticle concentration profiles showed sharp adsorption fronts in the macroporous resin, and more diffuse fronts in the dextran-grafted material.

\subsubsection{The Basis for Mass Transfer in Polymer-grafted Resins}

While both macroscopic and microscopic experiments demonstrate that polymergrafted resins can enhance protein adsorption properties relative to macroporous resins, the molecular details of adsorption and transport in these composite matrices are not fully understood. [15] For example, different types of proteins have 
exhibited significant variations in the extent to which $D_{e, a p p}$ is enhanced in a dextran-grafted material, and in the range of ionic strength at which the enhancements are observed. [20] Other studies have shown that altering the graft density and charge content within polymer-grafted resins can have very different effects on adsorption, depending on the protein. $[23,24]$ The physical bases for these variations are not fully understood, but presumably depend on the molecular properties of the protein and adsorbent, and how these species interact. A detailed understanding of how molecular properties affect macroscopic adsorption behaviors would be very useful for selecting optimal resins and operating conditions to separate a given protein most efficiently.

The enhancement of transport in polymer-grafted IEC resins has previously been explained by the possibility that protein molecules interacting with the charged polymer grafts retain diffusional mobility. $[9,20]$ Since the concentration of these molecules is expected to be higher than in free solution as a result of favorable electrostatic interactions with the charged polymers, transport should occur faster in the polymer-grafted pore compared to in an open macropore because the higher concentration gradient provides a greater driving force for diffusion. For conditions where protein binding is highly favorable, the following relationship can be used to relate $D_{e, a p p}$ to $D^{\prime}:[5,9]$

$$
D_{e, a p p} \sim D^{\prime} \frac{q^{*}}{C}
$$

where $q^{*}$ is the total adsorbed protein concentration at equilibrium. This expression shows that the transport rate in a solid diffusion-controlled material is determined by both the adsorbed phase diffusivity and the partitioning of proteins between the adsorbed and solution phases. Therefore, even if $D^{\prime}$ is low, 
the effective diffusivity can be high if the material provides a sufficiently high binding capacity to compensate for the slow diffusion in the adsorbed phase.

While Eq. 1.3 provides an approximate model for transport in a solid diffusioncontrolled material, this description is incomplete for the polymer-grafted materials discussed above, in which proteins adsorption is thought to occur both within the charged polymer-filled pore space and on the charged underlying surface. Eq. 1.3 does not distinguish between protein molecules in these two different "phases", and thus, $D^{\prime}$ is an effective molecular diffusivity incorporating the differing behaviors of protein molecules on and off of the pore surface. A more realistic description is given by the parallel diffusion mass transfer model with the following flux expression,

$$
J=-D_{p} \nabla c_{p}-D_{s} \nabla q_{s}
$$

where $D_{p}$ and $D_{s}$ are the protein diffusivities in the pore space and on the surface, respectively, and $c_{p}$ and $q_{s}$ are the adsorbed protein concentrations of these respective phases, which are assumed to be in equilibrium. However, this more detailed mass transfer model currently has limited use experimentally due to the challenges of distinguishing between protein bound to polymers versus to the surface. Sophisticated imaging techniques such as TIRF microscopy, which has been utilized to track individual proteins in various environments, $[25,26]$ will be needed to measure the partitioning of protein between different phases within a porous particle and local diffusivities in these phases. 


\subsubsection{Opportunities for Study by Molecular Modeling}

Computational modeling is one approach to studying the molecular details of protein adsorption in porous resins. Molecular dynamics (MD) simulation, in which the motion of a system of molecules is simulated over time according to Newton's equation of motion, has been used to study protein adsorption and transport on various types of chromatographic surfaces, [27, 28] including surfaces representative of macroporous and polymer-grafted IEC resins. [29-31] Such molecular-level simulations can be used to probe phenomena the partitioning and diffusion of individual protein molecules with different phases. These behaviors influence the adsorption properties measured on macroscopic scales, as well as the mechanism by which mass transfer occurs. However, because protein adsorption is measured over much longer time and length scales in the laboratory than can currently be accessed by molecular simulation, multi-scale modeling is necessary to predict adsorption properties on macroscopic scales based on information obtained from molecular simulation.

\subsection{Scope and Outline of this Dissertation}

This dissertation presents research on the use of multiscale modeling to study how protein adsorption can be enhanced in polymer-grafted IEC resins. A fundamental understanding of the molecular basis for enhanced adsorption properties in these systems would be valuable for the design of efficient protein chromatography steps, which could ultimately reduce the costs of producing of biopharmecuticals. The modeling approach developed in this work consists of molecular dynamics simulations of protein molecules within IEC pores with different properties, as well as simulations of mass transfer over longer scales. Chapter 2 
details the development, parameterization, and validation of our modeling approach. This work has recently been published in AIChE Journal, [32] and draws upon our earlier findings on the effects of MD thermostat algorithms on kinetic properties, published in Journal of Chemical Theory and Computation. [33] Chapter 3 describes a study recently published in Langmuir [34] on how varying the properties of a polymer-grafted IEC resin affects lysozyme's adsorption as predicted by multiscale modeling. Chapter 4 describes a study on how properties of the protein effect adsorption, and how the proteins interact with the charged polymers on a molecular level, as predicted by simulations. 


\section{Chapter 2}

\section{Model Development}

\subsection{Introduction}

This chapter describes the development of a multiscale model to elucidate protein adsorption and transport behaviors in ion-exchange chromatography (IEC) adsorbent particles that have either an open pore structure or charged dextran polymers grafted into the pores. Molecular dynamics (MD) simulation is used to determine protein diffusion and partitioning in different regions of the adsorbent pore, and these outputs are used in numerical simulations of mass transfer to determine the intraparticle protein concentration profile and the mass transfer rate. Modeling results indicate that, consistent with experimental observations, protein transport can be faster in the polymer-grafted material compared to the open pore case. This occurs when favorable partitioning of protein into the polymer-filled pore space is combined with relatively high protein mobility within this region. 


\subsubsection{Background}

Various multiscale models have been used to study protein adsorption in macroporous resins as a function of molecular-level details. [35] Lenhoff has developed a model applicable to IEC systems governed by a parallel diffusion mechanism (in which proteins diffuse on the pore surface as well as within the pore fluid), that predicts the apparent pore diffusivity based on adsorbent structural properties, protein size and binding capacity, operating conditions, and chromatographic retention. [36] The model predicts a significant increase in $D_{e, a p p}$ of lysozyme in a commercial macroporous adsorbent as the salt concentration is increased. This trend is consistent with experiments by Dziennik et al. for lysozyme adsorption in the cation exchanger SP Sepharose FF. [37] However, it is inconsistent with the results of Ubiera and Carta who found a constant diffusivity of $D_{e, a p p}=1.8 \pm 0.3 \times 10^{-7} \mathrm{~cm}^{2} / \mathrm{s}$ for the same system at ionic strengths between 0.02 and $0.12 \mathrm{M}$. [38] The reasons why the results of Dziennik et al. and those of Ubiera and Carta are different are not known. One possibility is that Dziennik et al. used a batch method where the protein solution concentration varied and the kinetics were determined by material balance, while Ubiera and Carta used a radiotracer method which provided direct measurements at a constant protein concentration. Ubiera and Carta also found that $D_{e, a p p}$ of lysozyme decreases rather than increases with ionic strength in the cation exchanger SP Sepharose XL, which has the same backbone matrix as SP Sepharose FF but contains charged grafted dextran polymers. [38] Riccardi and coworkers also used multiscale modeling to predict intraparticle protein concentration profiles in an adsorbent governed by ordinary pore diffusion, based on molecular dynamics (MD) simulation results for the spatial distribution of charged ligands immobilized within the adsorbent. In addition to these multiscale studies, MD has been used extensively to study 
the structural and energetic details of peptide and protein molecules interacting with various types of chromatography adsorbents and ligands, [27, 28] including charged surfaces [29, 39-41], charged polymers, [30, 31] and multimodal chromatography ligands. [42]

While the multiscale models described above are applicable to macroporous adsorbents, a more generalizable approach is needed to predict protein adsorption in complex systems for which the underlying transport mechanism is not wellunderstood, such as polymer-grafted adsorbents. Therefore, we have developed a multiscale model for studying adsorption behaviors in IEC systems with different pore architectures based on molecular-level details. The approach includes MD simulation used to study the diffusion and partitioning of protein molecules in different locations within an adsorbent pore. From this partitioning behavior the adsorption equilibria can be predicted. The modeling uses numerical simulation of mass transfer to predict the adsorption kinetics over experimentally-relevant length and time scales, as a function of the molecular behaviors observed from MD.

This chapter focuses on two objectives directed towards developing the multiscale model. The first is to determine how the molecular behaviors of the protein interacting with the adsorbent affect the overall adsorption kinetics. The second is to determine which molecular details of the model lead to macroscopic adsorption behaviors that are consistent with the experimental results described above for lysozyme in macroporous and dextran-grafted IEC particles. [9] In this chapter, we describe initial steps towards being able to predict adsorption behaviors as a function of the protein and adsorbent's molecular-level details, for macroporous as well as polymer-grafted ion exchangers. 


\subsection{Development of molecular models}

\subsubsection{Overview}

We perform MD simulations of protein molecules in both an open macropore and a polymer-grafted pore environment in order to determine the diffusion and partitioning of the protein based on the system's molecular-level properties. A coarse-grained (CG) approach in which multiple atoms are lumped into individual interaction sites (or "beads") is used to efficiently simulate large ensembles of protein molecules in order to measure diffusivities and partitioning behaviors with low statistical error. Rather than deriving the CG model from a more detailed atomistic model, we use a hypothesis-driven approach, in which we include in the model only the components and interactions that we hypothesize control the protein-adsorbent interactions that govern macroscopic adsorption behaviors. The components are the lysozyme protein molecules, the sulfonate ligands on the agarose pore surfaces, and the charged dextran grafts (for the polymer-grafted system). Fig. 3.1 shows snapshots from MD simulations of the macroporous and polymer-grafted systems with lysozyme. Explicit water molecules and solution ions are not included, however, their effects on molecular diffusion and electrostatic interactions are represented implicitly, as discussed in Sec. 2.2.5 


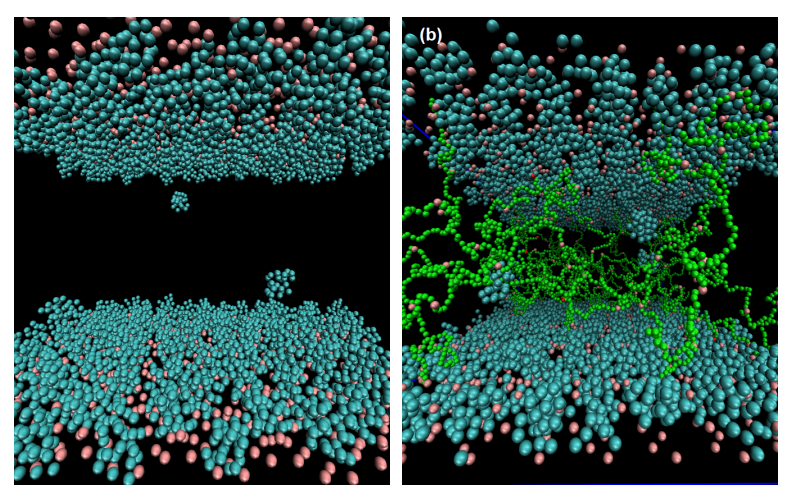

Figure 2.1: Snapshots from equilibrated MD simulations of lysozyme within an idealized pore of a cation-exchange particle, with either an open pore structure (a), or with charged dextran polymers grafted onto the pore surface (b). Both adsorbents are near saturation. Lysozyme, dextran, and sulfonate groups are shown in blue, green, and pink, respectively.

Our molecular models have an intermediate level of coarse-graining compared to previous simulation studies on protein adsorption in charged polymer-grafted systems. For instance, we use a CG protein model with a higher-resolution structure and charge distribution than the cubic lattice model of Johansson and Van Alstine, in which the protein is represented as a sphere with its net charge placed in its center. [43] Higher resolution of these molecular properties will be required to eventually study how adsorption and diffusion behaviors may vary for different protein species. However, our coarse-graining approach and implicit representation of the solvent provide less detail than the models used by Riccardi and co-workers. [30, 44] We hypothesize that the factors controlling adsorption and transport of an ensemble of protein molecules in the charged polymer-grafted pore involve only larger-scale molecular properties such as charge distribution and protein flexibility. Therefore, we employ simpler CG models in order to ef- 
ficiently study a multi-dimensional variable space with large-scale simulations. Relatively long time scales and large systems with multiple protein and polymer molecules are required to measure protein concentrations and diffusivities with sufficient precision to compare to experiments.

\subsubsection{Justification of coarse-grained models}

Although our CG models are significant approximations to the physical systems, their use for studying how molecular details qualitatively affect adsorption and diffusion is justified by the properties of the physical IEC systems. Due to the high hydrophilicity of the underlying agarose surface and the dextran polymers, protein adsorption is expected to be dominated by electrostatic attraction to the charged ligands. Previous MD simulations have demonstrated the dominant role of electrostatics in protein adsorption onto a charged hydrophilic surface, which may perturb but not completely displace the water layers on the surface, [45] Therefore, the free energy of desolvation associated with protein adsorption, which is not considered in these implicit solvent simulations, should not affect the qualitative trends we observe in protein partitioning with respect to adsorbent charge content and the strength of electrostatics. We note that this approach would not be appropriate for modeling hydrophobic interaction or mixed-mode chromatography systems, as the entropy increase associated with water moving from the protein interface to the bulk would be dominant for these cases. [46]

Another important approximation made in this work is modeling the screening of charged interactions with a modified Coulombic potential rather than with explicit ions. This approach is justified for our systems because the IEC adsorption experiments that we are modeling are conducted in a range of relatively low ionic strengths (below $200 \mathrm{mM} \mathrm{NaCl}$ ). [20] At low salt concentrations, counter 
ions screen electrostatics by interacting directly with charged solutes, and only at higher concentration do they compete with solutes for interfacial water. [47] Therefore, we approximate their effects by simply scaling electrostatic interactions by the dielectric $\epsilon_{r}$, as described above. As shown in Figures 3.2 and 3.3, the parameters $\epsilon_{r}=10$ and $\epsilon_{r}=15$ lead to relatively high and low levels of protein adsorption, respectively. These parameters also lead to very similar equilibrium polymer structures in the absence of protein. At very high salt concentrations in which no protein binding occurs, the polymers are expected to collapse somewhat due to reduced repulsion between charged ligands. [9] However, it is not clear whether the charged dextrans modeled here would exhibit significantly different conformations for weak versus strong binding conditions, or similar structures as predicted by our simulations.

\subsubsection{Comparison with Atomistic Simulation}

Finally, we note that modeling cooperative properties between multiple proteins and polymers requires a trade off between molecular detail, system size, and computational efficiency. The use of a CG model, an implicit representation of the solvent, and simple interaction potentials improves the computational efficiency of the MD simulations by approximately 4 orders of magnitude as compared to using a model with full atomistic detail. To illustrate, on a standard 8-core desktop, a simulation of a CG model of the polymer-grafted pore and a high protein loading ran at $0.16 \mathrm{hrs} / \mathrm{ns}$, while an atomistic simulation of approximately 1.3 million water molecules (which would comprise most of the overall computation cost) with the same box dimensions used for the CG case ran at $1600 \mathrm{hrs} / \mathrm{ns}$.

While simulations of dextran interacting with the protein based on more detailed models would be useful for validation of the CG models, our initial at- 
tempts to study these systems with fully atomistic MD simulations have been limited by the accuracy of current force fields for carbohydrates. We prepared simulations of a functionalized dextran chain with a length of 100 monomers (approximately $16 \mathrm{kDa}$ ), and 2, 4, or 8 of these monomers sulfonated. The online tool GLYCAM Web [48] was first used to obtain an initial structure and force field parameters for 4-member dextran segments from which larger chains were built. [48, 49] These 4-membered segments were composed of D-glucose units connected by either $\alpha-1,6$ linkages for the main chain, or $\alpha-1,3$ linkages for side chain branches. All side chain branches consisted of one monomer. Code was then written to connect any combination of straight or branched 4-membered segments to form larger chains, and to functionalize the side chain branches with the spacer arm and sulfonate group used in the experimental systems. [50] These algorithms involved removing the necessary $-\mathrm{OH}$ and $-\mathrm{H}$ groups of the segments to be linked, and rotating the added segment to avoid steric clashes. Initial coordinates for the spacer arm and sulfonate group were obtained using the online tool PRODRG. [51] The GAFF force field was used to parametrize atoms in the spacer arm. [52] For atoms of the sulfonate ligand, force field parameters and partial charges were adapted from a previous study on the parametrization of alkylsulfonate ions. [53] A single chain was simulated in explicit solvent in a periodic box with a $20 \mathrm{~nm}$ box length. The systems included approximately 800000 particles (1 dextran chain, 266000 TIP3P water molecules and approximately 100 sodium and chloride atoms, corresponding to $20 \mathrm{mM}$ salt concentration). The simulations were run in parallel on 1152 CPUs on Kraken, a now depreciated supercomputing cluster of the National Institute for Computational Sciences, which provided approximately $10 \mathrm{~ns} /$ day of data.

MD simulations of the charged dextran chains appear to be of limited value 
because the polymers adopt an overly-collapsed equilibrium structure. This is evident in Fig. 2.2, which shows the progression of the chain from its extended initial conformation to a highly collapsed structure over a $5 \mathrm{~ns}$ period. Due to resource limitations, a converged estimate of the polymer's radius of gyration could not be obtained, but over the course of $5 \mathrm{~ns} R_{g}$ decreases from an initial value of over $10 \mathrm{~nm}$ to $2 \mathrm{~nm}$. By contrast, previous experiments suggests that 16 $\mathrm{kDa}$ dextran in dilute aqueous conditions should have a hydrodynamic radius of 5 to $6 \mathrm{~nm}$. [54] While these different physical properties do not allow for a direct comparison, the large discrepancy in the radii does suggest that the simulated chain is overly collapsed. Thus, this model for dextran is of limited use for studying lysozyme diffusion through a charged hydrogel-like environment. The observed behavior of the polymers may be related to unphysically weak waterpolymer interactions in the GLYCAM force field. 


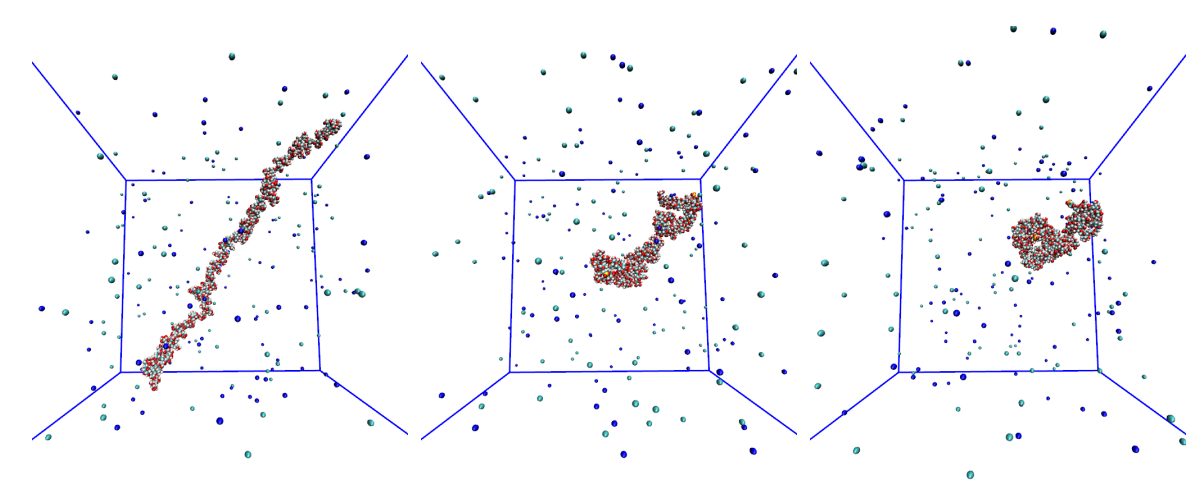

Figure 2.2: Snapshots of MD simulation of an atomistic sulfonated dextran chain, at times of $1.2 \mathrm{~ns}(\mathrm{a}), 3.7 \mathrm{~ns}(\mathrm{~b})$, and $6.2 \mathrm{~ns}$ (c). Solution ions are shown in blue shades, while water molecules are omitted for clarity.

\subsubsection{Parameterizing the CG Models}

The approach used to parametrize the molecular details of the CG models depends on the relative uncertainty in our information about these details. Details describing intrinsic material properties that are known with relatively low uncertainty are parametrized to reproduce these properties as directly as possible. For example, the size and structure of the protein molecule are parametrized based on the protein's crystal structure, under the assumption that the crystal structure is an appropriate approximation for the native structure in solution.

Model details that are not directly constrained by experimental data, such as the density of ligands on the surface, the charge content of grafted polymers, and the strength of electrostatic interactions under favorable binding conditions are tuned such that the model predicts adsorption behaviors that agree qualitatively with the following experimental behaviors for lysozyme under favorable binding conditions: 
1. a monolayer surface coverage of bound protein in the macroporous system

2. mass transfer controlled by diffusion in the liquid contained within the pore in the macroporous system (such that $D_{e, a p p} / D_{0} \approx 1$ ), and

3. an enhancement in $D_{e, a p p} / D_{0}$ for the polymer-grafted system.

The first criterion relates to the adsorption equilibria and is suggested by the fact that experimental adsorption equilibria are consistent with the Langmuir adsorption model, while the latter two criteria are related to both the adsorption equilibria and kinetics.

\subsubsection{Model Details}

All MD simulations were performed using GROMACS version 4.6.4. [55] The following sections describe details of the molecular models.

\section{Protein}

The most important aspects of the coarse-graining procedure are described here, while full details are provided in Appendix A. The protein model in this study is designed to approximate the charge distribution, excluded volume, and structural flexibility of lysozyme. Each protein molecule is modeled as an elastic network of CG beads, with each bead representing the excluded volume, mass, and net charge of 5 or 6 contiguous residues. Fig. 2.3 shows the mapping of the $C_{\alpha}$ atom of each lysozyme residue to the beads of the CG model with $n_{C G}=5$. This level of coarse graining provides both computational efficiency versus more detailed models, and provides a good approximation for lysozyme's surface charge distribution, as compared to that of a fully atomistic model. The elastic network consists of harmonic bonds defined between all particles separated by $1.5 \mathrm{~nm}$ 
or less. The average excluded volume of each particle and the strength of the harmonic potential of the elastic network model (applied uniformly to all bonds) are parametrized such that the CG model exhibits a radius of gyration and structural RMSD from its crystal structure that are consistent with the same properties of an atomistic model for lysozyme in explicit water. This same coarse-graining approach is applied to other protein molecules, as described in Chapter 4. 


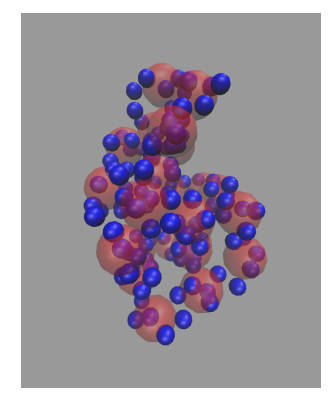

Figure 2.3: Mapping of $\alpha$-carbon atoms of lysozyme (shown in blue) to CG model with $n_{C G}=5$ particles per residue (shown in red).

\section{Pore Surface}

For both types of materials, the adsorbent pore is modeled as two parallel surfaces functionalized with charged ligands, as shown in Figs. 2.2c-4.1a. Two surfaces are modeled rather than a single surface in order to create a homogeneous charged polymer phase that more closely approximates what would exist in a dextrangrafted macropore with a diameter consistent with experimental observations. [9]. The idealized parallel surface model is used because while the physical agarose matrix is expected to have a highly heterogeneous pore structure based on TEM images, we hypothesize that lysozyme's adsorption and diffusion behaviors will be roughly independent of the large-scale curvature of the pore surface. Each sulfonate group functionalized to the surface is represented by a single CG particle with a fixed location and a charge of -1 . Because protein adsorption is dominated by electrostatics interaction with the charged ligands in these systems, [20] the underlying agarose is modeled implicitly by repulsive walls defined at the bottom and top of the simulation box. The excluded volume of both this wall and the charged particles is given by the potential $V_{L J}(r)=C_{12} / r^{12}$, where $\sigma$ and $\epsilon$ are the same as the LJ parameters used for the dextran monomers discussed in 
the next section.

The spatial distribution and density of surface ligands are not directly constrained by experimental data, but are expected to affect surface adsorption and diffusion behaviors. We vary the positions of the ligands relative to the bottom and top of the simulation box in order to partially model the expected heterogeneity of the underlying agarose structure. Any specific choice for these positions is somewhat arbitrary, as they are not constrained by experiment at the nanometer scale. Even a random structure requires choices for the distribution of heights of peaks relative to low points on the surface and the distribution of widths of these peaks. We therefore use a sinusoidal function to define the surface ligand positions, which allows us to systematically vary in a simple way just two length scales (peak height and separation), and obtain near-negligible surface diffusion, as is observed experimentally. The vertical position of each ligand, $z_{i}$, is defined by the $2 \mathrm{D}$ sine wave $z_{i}=A_{\text {surf }} \sin \left(x_{i} / T_{\text {surf }}\right) \sin \left(y_{i} / T_{\text {surf }}\right)$ with amplitude $A_{\text {surf }}$ and period $T_{\text {surf }}$. The coordinates $x_{i}, y_{i}$ are defined by a hexagonal packing arrangement, which maximizes the minimum lateral distance between neighboring ligands for a given surface density of ligands. Without additional information, such an arrangement is a reasonable choice, as during the functionalization process the charged ligands are likely to adopt a low energy configuration on the surface.

The density of ligands on the surface, $n_{S L} / S(S L$ : "surface ligand") cannot be estimated directly from the experimental charge content of a macroporous adsorbent, because the physical ligands functionalized to activated agarose monomers may not all be accessible to protein molecules. A series of multiscale simulations were used to determine appropriate values for $A_{\text {surf }}, T_{\text {surf }}$, and $n_{S L} / S$, as discussed in the Results section. 


\section{Charged Dextran Grafts}

Each charged polymer graft is modeled as a chain of CG beads, with each bead representing either a neutral dextran monomer, with a charge of 0 , or a monomer with a sulfonate ligand attached, with a charge of -1 . All polymer beads have a mass and excluded volume that approximate these properties for a glucose molecule. Bonds between adjacent monomers are described by the Finitely Extensible Non-linear Elastic (FENE) potential. Each chain is composed of 250 monomers, which approximates the size of the $40 \mathrm{kDa}$ dextran polymers used in the experimental material of interest. [9] Each chain is "grafted" to the pore surface by fixing the position of its first monomer at the position of a surface ligand, which is removed. The fixed monomers are arranged laterally in a hexagonal close packing arrangement.

The separation between each fixed monomer and its nearest neighbors is determined by the graft density, $\sigma=n_{\text {chains }} / S$, where $n_{\text {chains }}$ is the number of polymer chains on a pore surface and $S$ is the pore surface area. The charge content per polymer chain, $f_{P L}$, is simply defined as the fraction of charged monomers. In this study, $\sigma$ is equal to the estimated graft density of the experimental material, $\sigma_{e}$, [9] which corresponds to a graft density of 1 chain per $350 \mathrm{~nm}^{2}$. This graph density is estimated from $\rho_{\text {dex }} / S_{v}$, where $\rho_{\text {dex }}$ is the dextran content per adsorbent volume of the experimental material (18 $\mathrm{mg} / \mathrm{mlfor} 40 \mathrm{kDa}$ dextran grafts). [9] $S_{v}$ is the pore surface area per adsorbent volume, estimated as $S_{v}=2 \epsilon_{p} / r_{p o r e}$, which assumes the adsorbent has a porosity $\epsilon_{p}$ and idealized cylindrical pores with a uniform pore radius $r_{\text {pore }}$. Values of $\epsilon_{p}=0.9$ and $r_{\text {pore }}=19 \mathrm{~nm}$ are used in our calculations, consistent with the experimental data in Ref. 5. $f_{P L}$ is set to 0.05 , as this value is believed to be most consistent with the experimental material, assuming that the dextran chains have approximately $5 \%$ side chain branching $[56,57]$ and 
that each side chain branch is functionalized. [9] The polymer-grafted system in this study has a concentration of polymer-bound ligand, $c_{P L}$, of $4 \times 10^{-6} \mathrm{~mol} / \mathrm{ml}$. The concentration of surface-bound ligands is approximately $58 \times 10^{-6} \mathrm{~mol} / \mathrm{ml}$ for both the polymer-grafted and macroporous systems.

In order to measure the concentration of the free solution in equilibrium with the polymer-grafted pore, we model a void volume adjacent to the pore, as seen in the MD snapshot shown in Fig. 2.4. The polymer grafts are confined to the pore volume by two walls of purely repulsive particles whose positions are fixed at $y=0$ and $y=y_{\text {pore }}$, the boundaries between the pore and the void volumes. These particles interact only with the polymers, allowing protein molecules to access both the pore and the void. The particles are arranged in a hexagonal close packing arrangement with a separation of $1 \mathrm{~nm}$ from their nearest neighbors, and have the same $C_{12}$ parameter used for the agarose walls. Simulations of systems with different pore lengths ( $y_{\text {pore }}=34.8 \mathrm{~nm}$ and $69.6 \mathrm{~nm}$ ) exhibit timeaveraged protein concentrations within the polymer phase that are statistically indistinguishable, indicating that any effects of the repulsive walls on the polymer phase structure does not affect protein affinity for this phase.

The void region has a length of $3 y_{\text {pore }}$ such that the simulation box for the polymer-grafted system has lateral dimensions $x_{b o x}=40.2 \mathrm{~nm} \times y_{b o x}=104.4$ $\mathrm{nm}$. In the macroporous system it is assumed that the concentration of protein in the pore and not bound to the surface (referred to as the "pore space" in the remainder of this paper) would be in direct equilibrium with the free solution. Thus, in these systems $C$ is estimated within the pore space and the void region is not included, such that the simulation box has lateral dimensions $x_{\text {box }}=40.2$ $\mathrm{nm} \times y_{b o x}=34.8 \mathrm{~nm}$. Both systems have a box height of $z_{b o x}=28.0 \mathrm{~nm}$, and periodic boundary conditions are applied in the $x$ and $y$ dimensions. 


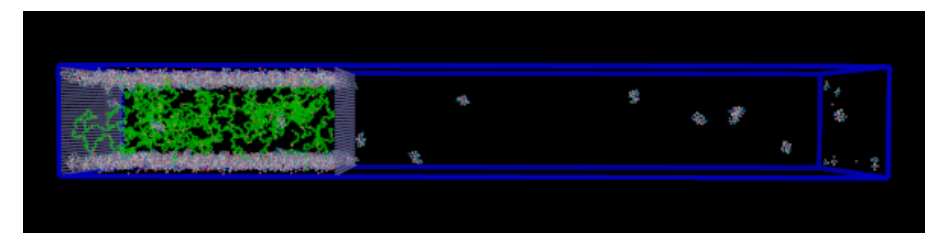

Figure 2.4: Snapshot from an equilibrated MD simulation of lysozyme in a polymer-grafted system. The void region adjacent to the adsorbent provides a control volume in which the free solution concentration, $C$, in equilibrium with the adsorbed phase can be measured.

\section{Solvent environment}

The system is evolved over time with the Langevin equation of motion using a temperature coupling strength, $\tau_{T}$, that maintains the system's average temperature at $T=300 \mathrm{~K}$. In simulations used to measure adsorption equilibria, $\tau_{T}=100 \mathrm{ps}$, as this relatively weak coupling accelerates the equilibration process. In simulations used to measure protein diffusivities, $\tau_{T}=0.7 \mathrm{ps}$, in order to approximate the viscous effects of a fictitious solvent on protein dynamics. [33] Electrostatic interactions are described by a modified Coulombic potential, with a dielectric constant $\epsilon_{r}$ applied to charged interactions separated by $r<r_{\text {Coulomb }}$, where $r_{\text {Coulomb }}$ is 2.58 times the average radius of a CG protein bead. A constant dielectric environment is assumed for charged interactions at distances beyond $r_{\text {Coulomb. }}$. The inner dielectric is set to $\epsilon_{r}=10$ or $\epsilon_{r}=15$ to model relatively strong or weak binding conditions, respectively, corresponding to low and high ionic strengths. All MD simulations were performed using GROMACS version 4.6.4. [55] 


\subsection{Data Analysis Methods}

\subsubsection{Adsorption isotherms}

The equilibrium adsorption behavior is determined by running MD simulations of the adsorbent pore with various protein loadings, $N$, and measuring the local protein concentrations in different regions of the pore at equilibrium. Although equilibrium concentrations could be measured using semigrand canonical MD simulation in which the total number of particles is constrained and the number in each region fluctuates, this ensemble is not currently supported in the GROMACS code used for this work, and the non-standard molecular models employed here are not implemented in the codes that do support this ensemble.

Each simulation of a particular adsorbent type, electrostatic strength, and protein loading is initialized by incrementally inserting protein molecules into the simulation box at random locations and orientations, followed by steepest descent energy minimization. The majority of molecules are initialized in the interior region of the pore, such that they to not interact with the charged surface. Following energy minimization, the system is equilibrated in the NVT ensemble with $\tau_{T}=100 \mathrm{ps}$ used to accelerate the dynamics, and thus enhance the equilibration process. The simulation time is sufficiently long for the free solution protein concentration to reach equilibrium with the surface and polymer regions, as discussed below.

Three different local concentrations are measured, corresponding to the three distinct types of protein adsorption behaviors that are observed in the MD simulations. A protein molecule whose center of mass is within a distance $r_{\text {surf }}=3$ $\mathrm{nm}$ of a surface ligand is considered to be surface bound at a given time. This value of $r_{\text {surf }}$ was determined by considering the distribution of distances be- 
tween a protein molecule and the nearest surface ligand at equilibrium, as shown in Fig. 2.5. For all tested electrostatic strengths, $r_{\text {surf }}=3 \mathrm{~nm}$ encompasses the peak in the distribution at short separations corresponding to surface-bound protein, and excludes molecules not bound to the surface with longer separations. The concentration of surface-bound protein on a particle volume basis, $q_{s}$, is calculated as:

$$
q_{s}=\frac{\left\langle N_{s}\right\rangle}{S} m_{p r o t} S_{v}
$$

where $\left\langle N_{s}\right\rangle / S$ is the time-averaged number of surface-bound protein molecules per unit surface area and $m_{\text {prot }}$ is the protein molecular mass. The surface area over which $N_{s}$ is measured is defined at a distance of $\Delta y=8 \mathrm{~nm}$ away from the ends of the adsorbent in the $y$ dimension, to avoid unintended effects of the repulsive walls of the polymer-grafted system on surface adsorption.

A second type of molecular behavior is that of protein molecules within the pore space and not bound to the surface. The concentration of these molecules is defined as

$$
c_{p}=\frac{\left\langle N_{p}\right\rangle}{S} m_{p r o t} S_{v}
$$

where $\left\langle N_{p}\right\rangle$ is $1 / 2$ the time-averaged number of protein molecules within the pore space at a distance of $r_{\text {surf }}=3 \mathrm{~nm}$ or more from the nearest surface ligand. The factor of $1 / 2$ is used because like $q_{s}, c_{p}$ is predicted based on the number of protein molecules per unit surface area $S$, and the top and bottom planes of the system are considered separate surfaces. For the polymer-grafted system, $\left\langle N_{p}\right\rangle$ includes protein molecules that interact directly with ligands on the polymer grafts as well as those that do not interact. In both systems, the total concentration of protein 
in the adsorbent includes protein molecules on the surface as well as those in the pore space, and is defined as $\hat{q}=q_{s}+c_{p}$.

Finally, a third type of behavior is that of protein molecules in the simulated free solution or in equilibrium with a hypothetical free solution, whose concentration is calculated as:

$$
C=\frac{\left\langle N_{\text {free }}\right\rangle m_{\text {prot }}}{V_{\text {free }}}
$$

where $\left\langle N_{\text {free }}\right\rangle$ is the time-averaged number of molecules in a volume $V_{\text {free }}$ where proteins are unaffected by surface ligands or repulsive walls. In the macroporous system, $V_{\text {free }}$ is defined in the pore space at a distance $\Delta z=8 \mathrm{~nm}$ from $z=0$ and $z=z_{b o x}$ to avoid the effects of the charged surfaces. In the polymer-grafted system, $V_{\text {free }}$ is defined in the void region adjacent to the adsorbent, at a distance $\Delta z=8 \mathrm{~nm}$ from $z=0$ and $z=z_{b o x}$, and at a distance $\Delta y=8 \mathrm{~nm}$ from $y=y_{\text {pore }}$ and $y=y_{b o x}$, to avoid possible effects of the purely repulsive walls at the ends of the pore.

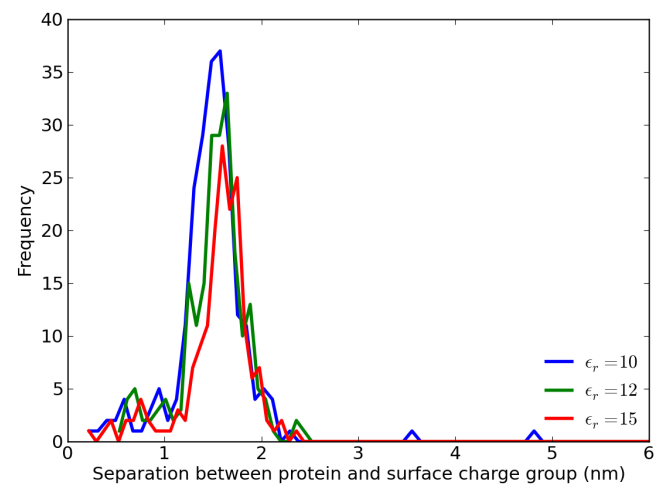

Figure 2.5: Histograms of the distance between the center of mass of protein molecules and their nearest surface ligand, used to determine the parameter $r_{a d s}$ that defines when a molecule is adsorbed on the surface. 
The equilibrium value of each local concentration is determined by averaging its time series over a period of 480 ns or longer over which $C$ exhibits stable oscillations about its mean. The time required to reach this equilibrium varies from $200 \mathrm{~ns}$ to $2000 \mathrm{~ns}$, and generally is longer for systems with grafted polymers, high protein loadings, and low $\epsilon_{r}$. The standard error in the mean for each

concentration is calculated as $\sigma / \sqrt{\left(n_{e}-1\right)}$, where $\sigma$ is the standard deviation of the equilibrated portion of the time series and $n_{e}$ is number of uncorrelated samples in that region. $n_{e}=n /\left(2 \tau_{A C F}\right)$, where $n$ is the original number of samples and $\tau_{A C F}$ is the decay constant of an exponential function fit to the autocorrelation function of the time series of concentration $C$ after equilibration.

\subsubsection{Molecular diffusivities}

Average molecular diffusivities of protein adsorbed to the surface, $D_{s}$, and within the pore space (either open or polymer-filled), $D_{p}$, are determined for each type of adsorbent and electrostatic strength. The diffusivities are measured from an MD simulation with a coupling strength $\tau_{T}=0.7 \mathrm{ps}$ and a protein loading $N$ that leads to $C=1-3 \mathrm{mg} / \mathrm{ml}$ at equilibrium, i.e. a nearly saturated adsorbed phase.

The diffusivity of a protein molecule over a given time window is determined from the slope of its $N_{d i m}$-dimensional mean squared displacement (MSD) according to the Einstein relation:

$$
2 \tau D=\frac{1}{N_{\text {dim }}}\left\langle\left|r_{i}(t+\tau)-r_{i}(t)\right|^{2}\right\rangle
$$

where $\tau$ is the offset time, the braces denote averaging over multiple reference times $t$, and $r_{i}$ is the molecule's center of mass coordinate. Our MD simulations show that protein molecules not adsorbed to the surface, either in an open 
macropore or within the polymer-filled pore space, exhibit isotropic diffusion, while molecules on the surface are restricted to the $x y$ plane. Therefore, 3- and 2dimensional MSD curves are used to evaluate the diffusivities of molecules with these respective adsorption behaviors.

Whether or not a protein molecule is surface-adsorbed also determines the length of the trajectory used to calculate the MSD, $\Delta t_{m s d}$, and the region of the MSD over which $D$ is estimated, as discussed in the Results section. The ensemble-averaged $D_{s}$ and $D_{p}$ are determined by averaging the MSD curves of all molecules that remain in the same adsorption state (i.e. on or off of the surface) for the entire period $\Delta t_{m s d}$, and evaluating the slope of the ensemble-averaged MSD. Only including molecules that remain in the same adsorption state for the entire $\Delta t_{m s d}$ avoids bias from rare events in which a molecule desorbs or readsorbs to the surface. The standard error of the mean of $D_{s}$ and $D_{p}$ is estimated by bootstrapping with 50 bootstrap repetitions.

\subsection{3 multiscale simulation of adsorption kinetics}

Numerical simulations of diffusional mass transfer are used to predict the effective protein transport rate as a function of properties that cannot be directly measured by experiment, including the diffusivities of protein molecules in distinct regions of the pore and the partitioning of protein between those regions. The following conservation equation and initial and boundary conditions describe transient 
adsorption into a spherical particle of radius $R$ over time $t$ and radial distance $r$ :

$$
\begin{gathered}
\frac{\partial c_{p}}{\partial t}+\frac{\partial q_{s}}{\partial t}=\frac{1}{r^{2}} \frac{\partial}{\partial r}\left[r^{2}\left(D_{p} \frac{\partial c_{p}}{\partial r}+D_{s} \frac{\partial q_{s}}{\partial r}\right)\right] \\
q_{s}=f\left(c_{p}\right) \\
r=0: \frac{\partial c_{p}}{\partial r}=0 \\
r=R: c_{p}=f\left(C_{0}\right) \\
t=0: c_{p}=q_{s}=0
\end{gathered}
$$

where $f\left(c_{p}\right)$ describes the local equilibrium between protein on the surface and in the pore space, i.e. the $q_{s}$ versus $c_{p}$ adsorption isotherm. As discussed in the Results section, for each system this isotherm is well described by the Langmuir model given in Eq. 2.8 with parameters $q_{m, s}$, the saturation capacity of the surface (defined on an adsorbent volume basis), and $K_{s}$, the equilibrium constant for protein adsorption from the pore space onto the surface (defined on a free solution volume basis). $C_{0}$ is the protein concentration of the extra-particle bulk solution, assumed to be constant at $2.0 \mathrm{mg} / \mathrm{ml}$. The boundary condition $c_{p}=f\left(C_{0}\right)$ at $r=R$ is determined from the local equilibrium between protein in the bulk and in the pore space, i.e. the $c_{p}$ versus $C$ isotherm. As described in the Results, in the polymer-grafted systems this local equilibria is described by the Langmuir model given in Eq. 2.9, with a saturation capacity $c_{p, m}$ and equilibrium constant $K_{p}$. In the macroporous system, the bulk-pore space equilibria is described by $c_{p}=K_{D} C$ (Eq. 2.10). The isotherm parameters as well as $D_{p}$ and $D_{s}$ are determined from MD simulation.

The numerical simulations are performed by expressing Eq. 2.5 in terms of $c_{p}$ based on the $q_{s}-c_{p}$ local equilibria, and solving by the method of lines. [58] The 
particle-averaged protein concentration at a given time is then calculated as:

$$
\bar{q}=\frac{3}{R^{3}} \int_{0}^{R} r^{2} \hat{q}(r) d r
$$

where $\hat{q}(r)$ is the total protein concentration at position $r$ within the simulated particle, and includes molecules both on and off of the surface.

In order to quantify the adsorption rate predicted by a simulation, following the procedure of Stone and Carta, we define an apparent effective pore diffusivity, $D_{e, a p p}$, as the pore diffusivity that would give the same adsorption kinetics as that obtained experimentally for a macroporous matrix with a rectangular adsorption isotherm and conditions where $q_{s} \gg c_{p}$. [9] In this case, the batch uptake curve $(\bar{q}$ vs. $t)$ is given by the following equation:

$$
\frac{1}{2}-\frac{1}{3} \frac{\bar{q}}{\hat{q}^{*}}-\frac{1}{2}\left(1-\frac{\bar{q}}{\hat{q}^{*}}\right)^{\frac{2}{3}}=\frac{C_{0}}{\hat{q}^{*}} \frac{D_{e, a p p} t}{r_{p}^{2}}
$$

where $\hat{q}^{*}$ is the protein concentration in the adsorbent particles at equilibrium. $D_{e, a p p}$ is estimated by fitting the simulated batch uptake curve with Eq. 2.7, by minimizing the squared error between the fitted curve and the data over the region from $t=0$ to the time at which $\hat{q}=\hat{q}^{*}$.

\subsection{Model parametrization}

\subsubsection{Effects of protein diffusion and partitioning on macroscopic adsorption kinetics}

A series of mass transfer simulations based on Eq. 2.5 were performed in order to study the relationships between molecular-level properties and the effective 
transport rate, which helps guide the parametrization of molecular model details that are not directly constrained by experimental data. The molecular-level properties of interest include the protein diffusivities $D_{s}$ and $D_{p}$, the favorability of protein adsorption from the pore space (which may or may not include polymer) onto the surface, quantified by $K_{s}$, the saturation capacity of the surface, $q_{m, s}$, and for the polymer-grafted system, the Langmuir isotherm parameters $K_{p}$ and $c_{p, m}$ for partitioning from the bulk into the polymer-filled pore space. In these systems, the affinity of protein for the charged polymers impacts both the partitioning between the surface and the pore space, and between the pore space and the bulk, through the parameters $K_{s}$ and $K_{p}$, respectively. The partitioning between the polymer-filled pore space and the bulk only affects the solution of Eq. 2.5 through the value of $\left.c_{p}\left(C=C_{0}\right)\right|_{r=R}$, i.e. the concentration of protein in the pore space at the edge of the particle that is in equilibrium with a bulk of constant concentration $C_{0}$. We thus use this as our independent variable rather than varying $K_{p}$ and $c_{p, m}$ individually. For all molecular-level properties, the parameter ranges tested are representative of the protein behaviors observed in trial MD simulations. The overall transport rate is quantified by the $D_{e, a p p}$ fit to the simulated batch uptake curve.

Fig. 2.6 shows how the transport of lysozyme in a macroporous system depends on the diffusion and adsorption of protein on the surface as predicted by this model. The predicted $D_{e, a p p} / D_{0}$ is plotted as a function of $K_{s}$ on the $x$ axis, $D_{s}$ on the $y$ axis, and $q_{m, s}$ in the different subplots. In these simulations $D_{p}=D_{0}$, which assumes molecules in the open pore space diffuse as they would in free solution, and $\left.c_{p}\right|_{r=R}=2.0 \mathrm{mg} / \mathrm{ml}$, which assumes the pore space concentration at the particle edge has the same concentration as the bulk. 

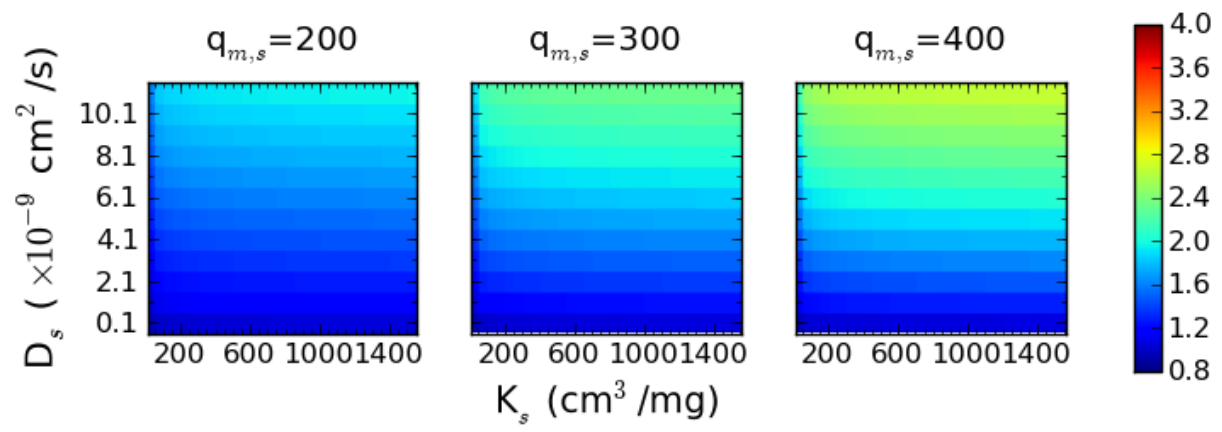

Figure 2.6: $D_{e, a p p} / D_{0}$ for lysozyme in a macroporous system is predicted from numerical simulation of mass transfer based on different molecular behaviors and plotted as a function of $D_{s}, K_{s}$, and $q_{m, s}$ (units of $\mathrm{mg} / \mathrm{cm}^{3}$ ), assuming $D_{p}=D_{0}$ and $\left.c_{p}\right|_{r=R}=C_{0}$ for protein molecules in an open macropore. According to this model, the effective transport rate increases primarily with the diffusivity of surface-bound protein $D_{s}$, as shown in the upper regions of each plot, although the surface capacity $q_{m, s}$ and favorability of adsorption to the surface $K_{s}$ have some affect on the effective transport rate.

In general, the transport rate is most dependent on the surface diffusivity $D_{s}$, though it has some dependence on the surface's saturation capacity $q_{m, s}$ and on the favorability of surface adsorption $K_{s}$, as well. For instance, at $q_{m, s}=400$ $\mathrm{mg} / \mathrm{ml}$, an increase in $D_{s}$ of approximately two orders of magnitude raises $D_{e, a p p} / D_{0}$ by approximately one order of magnitude, while a two order of magnitude increase in $K_{s}$ raises $D_{e, a p p} / D_{0}$ by only 10 to $25 \%$. Additionally, the effect of $K_{s}$ on $D_{e, a p p} / D_{0}$ depends only slightly on $D_{s} . \quad D_{e, a p p} / D_{0}$ is also somewhat dependent on $q_{m, s}$, increasing by up to $35 \%$ when $q_{m, s}$ is doubled. The effective transport rate is most consistent with that predicted by ordinary pore diffusion (at which $D_{e, a p p} / D_{0} \approx 1$ ) when $D_{s}$ is $1 \times 10^{-9} \mathrm{~cm}^{2} / \mathrm{s}$ or lower, for all $K_{s}$ and $q_{m, s}$ values tested. 
Fig. 2.7 shows that in the polymer-grafted system, faster transport is predicted as protein partitioning from the bulk into the pore space increases, and as the diffusion of protein within the pore space increases. Each subplot shows $D_{e, a p p} / D_{0}$ as a function of $K_{s}$ and $D_{p}$ on the $x$ and $y$ axes, respectively. $q_{m, s}$ increases in the subplots from left to right, $\left.c_{p}\right|_{r=R}$ increases from bottom to top, and $D_{s}$ is kept constant at $0.5 \times 10^{-9} \mathrm{~cm}^{2} / \mathrm{s}$, representing slow surface diffusion under strong binding conditions. $D_{e, a p p} / D_{0}$ is approximately a linear function of both $\left.c_{p}\right|_{r=R}$ and $D_{p}$ for fixed values of $K_{s}$ and $q_{m, s}$ over the tested variable space. The transport rate is approximately independent of $K_{s}$ and $q_{m, s}$ under these conditions, increasing by only $5 \%$ when $K_{s}$ is increased from $2 \mathrm{~cm}^{3} / \mathrm{mg}$ to $1000 \mathrm{~cm}^{3} / \mathrm{mg}$ (not shown). Additional simulations with different values for $D_{s}$ and with $\left.c_{p}\right|_{r=R}=20.0 \mathrm{mg} / \mathrm{ml}$, show that surface diffusion has a small effect on the overall transport rate in the polymer-grafted system compared to the macroporous system, as transport in the polymer-grafted adsorbent under favorable binding is dominated by the flux through the polymer-filled pore space. [32] 


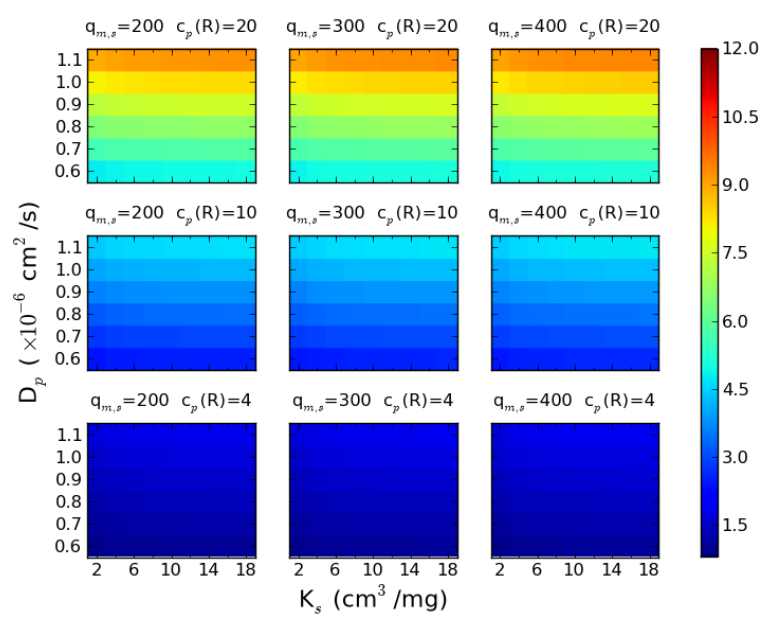

Figure 2.7: $D_{e, a p p} / D_{0}$ for lysozyme in a polymer-grafted system is predicted from numerical simulation of mass transfer based on different molecular behaviors and plotted as a function of $D_{p}, K_{s}, q_{m, s}$ (units of $\mathrm{mg} / \mathrm{cm}^{3}$ ), and $\left.c_{p}\right|_{r=R}$ (representing different levels of partitioning into the pore), with $D_{s}=0.5 \times 10^{-9} \mathrm{~cm}^{2} / \mathrm{s}$. According to this model, the effective transport rate is most enhanced when protein partitioning from the bulk into the pore is favorable, as seen in the upper subplots, and when diffusion in the polymer-filled pore is fast, as seen in the upper third of each subplot.

These simulations can be used to constrain parameter values for molecular model details that are not directly obtainable from experimental data. For example, Fig. 2.6 provides an upper bound on the surface diffusivity $D_{s}$ that the model surface can allow if the overall transport rate is consistent with that predicted by ordinary pore diffusion, the dominant mass transfer mechanism in a macroporous material. Fig. 2.7 provides lower bounds on the protein concentration within the polymer-filled pore space and the protein diffusivity $D_{p}$ in this region if the enhancement in the transport rate is consistent with that observed in the experimental dextran-grafted material. 


\subsubsection{Effects of molecular model parameters on protein diffusion and partitioning}

Table 2.1 lists molecular model parameters that lead to protein partitioning and diffusivities that are consistent with the experimental adsorption kinetics. These values are determined through a series of MD simulations of both adsorbent systems, as described in Appendix B. The estimated standard error in the mean of each property is given in parenthesis. As shown, an inner dielectric constant of $\epsilon_{r}=10$ and a polymer charge content of $n_{S L} / S=0.05$ lead to levels of protein partitioning and diffusion within the polymer-filled pore space that correspond to an effective transport rate of $D_{e, a p p} / D_{0}=6.1 \pm 0.6$, in qualitative agreement with experimental results for dextran-grafted systems at low ionic strength. In the macroporous system with $\epsilon_{r}=10$, a surface model with a charge density of 49 ligands $/ 100 \mathrm{~nm}^{2}$ and sine wave parameters of $A_{\text {surf }}=1 / 3 d_{\text {prot }}$ and $T_{\text {surf }}=d_{\text {prot }}$ lead to levels of protein partitioning and diffusion on the surface that correspond to $D_{e, a p p} / D_{0}=1.3 \pm 0.1$, which approaches the $D_{e, a p p} / D_{0}=1$ result expected for ordinary pore diffusion. Therefore, the production MD simulations of this study are performed with the adsorbent model parameters given above and with $\epsilon_{r}=10$ used to represent favorable binding conditions. 


\begin{tabular}{|c|c|c|}
\hline Model Detail (MD inputs) & Macroporous & Polymer-grafted \\
\hline$\epsilon_{r}$ & 10 & 10 \\
$n_{L} / n_{\text {poly }}$ & $\mathrm{n} / \mathrm{a}$ & 0.05 \\
$n_{S L} / S$ (ligands $\left./ 100 \mathrm{~nm}^{2}\right)$ & 49 & 49 \\
$A_{\text {surf }}$ & $1 / 3 d_{\text {prot }}$ & $1 / 3 d_{\text {prot }}$ \\
$T_{\text {surf }}$ & $d_{\text {prot }}$ & $d_{\text {prot }}$ \\
\hline Molecular behavior $(\mathrm{MD}$ outputs $)$ & Macroporous & Polymer-grafted \\
\hline$D_{s}\left(\times 10^{-6} \mathrm{~cm}^{2} / \mathrm{s}\right)$ & $1.3(0.2)$ & $0.6(0.4)$ \\
$D_{p}\left(\times 10^{-6} \mathrm{~cm}^{2} / \mathrm{s}\right)$ & $0.0012(0.0001)$ & $0.0008(0.0001)$ \\
$q_{m, s}(\mathrm{mg} / \mathrm{ml})$ & $282(<1)$ & $257(<1)$ \\
$K_{s}\left(\mathrm{~cm}{ }^{3} / \mathrm{mg}\right)$ & $56(4)$ & $11(13)$ \\
$c_{p}(r=R)(\mathrm{mg} / \mathrm{ml})$ & $2.2(<1)$ & $18.0(0.3)$ \\
\hline Transport rate & Macroporous & Polymer-grafted \\
\hline$D_{e, \text { app }} / D_{0}$ & $1.3(0.1)$ & $6.1(0.6)$ \\
\hline
\end{tabular}

Table 2.1: Summary of parameters for the molecular model details used in production MD simulations (MD inputs), the resulting protein diffusivities and adsorption isotherm parameters (MD outputs), and the effective transport rates predicted from numerical simulation based on these molecular behaviors. Numbers in parenthesis give the standard error of the mean.

Comparable transport rates can also be obtained using alternate parameters for the surface geometry. However, such geometries can affect the adsorption and diffusion behaviors of protein on the surface. The model with $A_{\text {surf }}=1 / 3 d_{\text {prot }}$ exhibits a single type of adsorption site, while a model with $A_{\text {surf }}=2 / 3 d_{\text {prot }}$ (i.e. larger peaks and troughs) and the same $T_{\text {surf }}$ and $n_{S L} / S=$ exhibits two types of 
adsorption sites, as shown in plots of the protein number density with respect to $z$ position in the pore, provided in Appendix B. The former (shallower peaks) leads to a relatively uniform distribution of surface diffusivities of individual protein molecules. However, the latter model with higher amplitude leads to rare events in which protein molecules diffuse up to an order of magnitude faster than the ensemble average of $D_{s}$. These fast-diffusing molecules generally interact with a single ligand located at high $z$ positions on the surface, while the majority of adsorbed molecules that interact with multiple ligands at lower positions. In this work, we use a molecular model that provides a more uniform distribution of surface diffusivities in order to be consistent with the macroscopic transport model that assumes a single average diffusion constant on the surface. The effects of surface heterogeneity on the heterogeneity of protein dynamics is a subject to be explored in later research.

Table 2 in Appendix B describes the effect of the surface geometry parameters on $D_{s}$. MD simulations were conducted with various surface geometries, including a completely smooth surface in which the ligands have a uniform height, and heterogeneous structures with different values of $A_{\text {surf }}$ and $T_{\text {surf }}$. The resulting $D_{s}$ values vary from $1 \times 10^{-8}$ to $1 \times 10^{-7} \mathrm{~cm}^{2} / \mathrm{s}$, with the fastest diffusivities generally observed when $T<d_{\text {prot }}$, as protein molecules cannot access the troughs of the surface. The smooth surface also leads to $D_{s}=1.7 \times 10^{-7} \mathrm{~cm}^{2} / \mathrm{s}$ for $\epsilon_{r}=12$. These results as well as the predicted $D_{e, a p p} / D_{0}$ vs. $D_{s}$ relationships shown in Fig. 2.6 demonstrate that some inhomogeneity in the surface structure is necessary to obtain a transport rate consistent with the pore diffusion mass transfer mechanism. 


\subsection{Effects of strength of electrostatics on adsorption behavior}

\subsubsection{Adsorption Equilibria}

MD simulations were performed using the adsorbent model parameters discussed above and different $\epsilon_{r}$ values in order to study how the strength of electrostatics affects the adsorption equilibria predicted by the model. Fig. 2.8a shows simulated adsorption isotherms of $\hat{q}$ versus $C$ for both types of adsorbents, with $\epsilon_{r}$ values of 10,12, and 15 representing low, moderate, and high ionic strengths, respectively. Each isotherm is fit with $\hat{q}=q_{s}+c_{p}$, based on the fitted $q_{s}$ versus $c_{p}$ and $c_{p}$ versus $C$ isotherm models described below. As shown, both adsorbents exhibit a lower adsorption capacity when electrostatic interactions are weaker, which is qualitatively consistent with experiments showing reduced lysozyme adsorption at high salt concentrations. [20] The adsorbents also become saturated at solution concentrations of $C=1$ to $3 \mathrm{mg} / \mathrm{ml}$, which is consistent with the experimental saturation concentrations. 

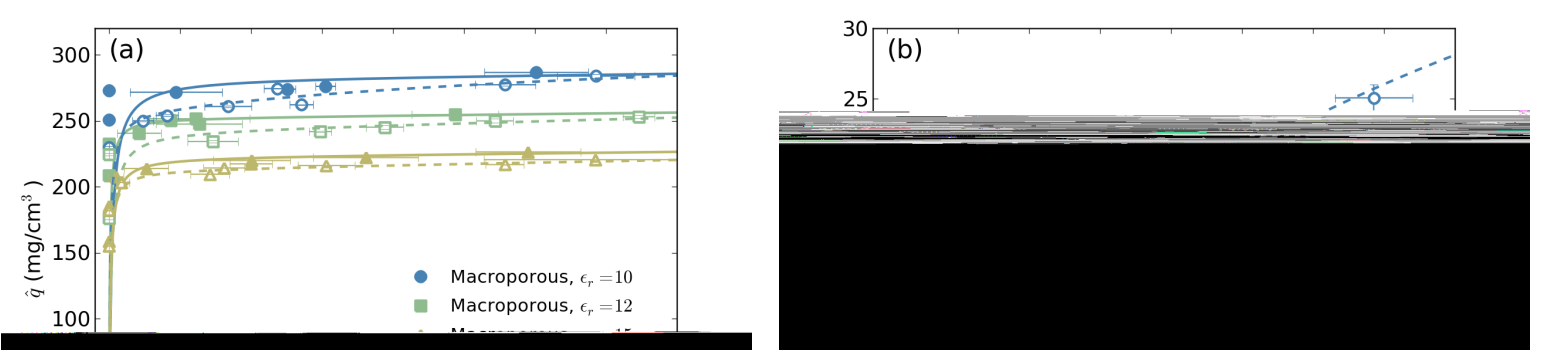

Figure 2.8: Adsorption isotherms predicted by MD simulation for (a) $\hat{q}$ versus $C$ and (b) $c_{p}$ versus $C$ show that when electrostatic interactions are weaker (at high $\epsilon_{r}$ values), the overall adsorption capacity decreases for both the macroporous (filled symbols) and the polymer-grafted (open symbols) systems. Protein partitioning into the polymer-filled pore also decreases as electrostatics become weaker. Error bars show the standard error of the mean concentrations.

The simulations do not predict an enhancement in the overall adsorption capacity of the polymer-grafted system versus the macroporous when electrostatics are strong. However, experiments conducted at low ionic strength found the capacity was only approximately 10 to $20 \%$ higher in dextran-grafted particles than in macroporous particles with a similar backbone and a slightly (6\% higher) total charge content. [20] Given the resolution of the model, this relatively small discrepancy is likely not a concern.

Nevertheless, the slightly lower than expected capacity of the polymer-grafted system can be understood by considering the local equilibria between $q_{s}$ and $c_{p}$ and between $c_{p}$ and $C$. The $q_{s}$ versus $c_{p}$ isotherms (not shown), which generally 
are very favorable and therefore are fit with the following Langmuir model:

$$
q_{s}=\frac{q_{m, s} K_{s} c_{p}}{1+K_{s} c_{p}}
$$

The macroporous systems generally exhibit higher surface capacities, as seen in the isotherms and in the fitted $q_{m, s}$ values reported in Table 2.2, because the polymer grafts occupy a non-negligible area on the surface and thus reduce the effective number of surface binding sites.

Fig. $2.8 \mathrm{~b}$ provides the $c_{p}$ versus $C$ isotherms, which show that the charged polymers can enhance protein partitioning from the bulk into the pore space when electrostatics are strong. However, this enhancement is negated by the reduction in surface adsorption due to the grafts, resulting in very similar total capacities for the two adsorbents. At high $\epsilon_{r}$, the protein shows little preference for the polymer-filled pore over the bulk. The fact that even with strong electrostatics, adsorption to the polymers is sufficiently low that it is negated by the reduced surface adsorption, whereas experiments show an overall enhancement due to the polymers, suggests that the actual dextran charge content may be greater than $5 \%$, that the actual protein may have a greater effective charge, that non-electrostatic forces might contribute moderately to protein-polymer binding, or some combination of all three. The $c_{p}$ versus $C$ isotherms are fit with the Langmuir model shown in Eq. 2.9 for the polymer-grafted systems, and with the linear model shown in Eq. 2.10 for the macroporous systems.

$$
c_{p}=\frac{c_{p, m} K_{p} C}{1+K_{p} C}
$$




$$
c_{p}=K_{D} C
$$

For macroporous systems, $K_{D}$ describes the equilibrium partitioning between the fictitious bulk solution and the open macropore. Slight deviations from a slope of $K_{D}=1$ result from the different control volumes used to calculate $c_{p}$ and $C$, as the former includes all molecules not bound to the charged surface, and the latter includes molecules that do not interact with the surface at all.

The isotherm models are fit to the $c_{p}$ versus $C$ and $q_{s}$ versus $c_{p}$ data by nonlinear regression, and uncertainties in the fitted parameters are estimated using a block bootstrap procedure. For each of 1000 bootstrap repetitions, an isotherm is constructed by evaluating the mean of concatenated, uncorrelated blocks of the original concentration time series for each protein loading used in the original isotherm. The block length is chosen as twice the decorrelation time of the instantaneous concentration. Each of these 1000 isotherms is fit with the appropriate model. The standard error in the mean of a given model parameter is estimated from the standard deviation of the 1000 predicted parameters. 


\begin{tabular}{|lllllll|}
\hline System & $\epsilon_{r}$ & $\begin{array}{l}q_{m, s} \\
(\mathrm{mg} / \mathrm{ml})\end{array}$ & $\begin{array}{l}K_{s} \\
\left(\mathrm{~cm}^{3} / \mathrm{mg}\right)\end{array}$ & $\begin{array}{l}c_{p, m} \\
(\mathrm{mg} / \mathrm{ml})\end{array}$ & $\begin{array}{l}K_{p} \\
\left(\mathrm{~cm}^{3} / \mathrm{mg}\right)\end{array}$ \\
\hline Macroporous & 10 & $282(<1)$ & $56(4)$ & - & - & $K_{D}$ \\
Macroporous & 12 & $252(<1)$ & $182(31)$ & - & - & $1.06(0.05)$ \\
Macroporous & 15 & $223(<1)$ & $99(10)$ & - & - & $1.06(0.10)$ \\
Polymer-grafted & 10 & $257(<1)$ & $11(13)$ & $64(7)$ & $0.20(0.03)-$ \\
Polymer-grafted & 12 & $243(<1)$ & $29(6)$ & $35(23)$ & $0.10(0.05)-$ \\
Polymer-grafted & 15 & $213(<1)$ & $74(7)$ & $7(<1)$ & $0.90(0.14)-$ \\
\hline
\end{tabular}

Table 2.2: Protein partitioning onto the surface and into a polymer-filled pore is greatest at $\epsilon_{r}=10$, at which electrostatics are strong. Adsorption isotherms measured from MD simulation are quantified by fitting data with either a Langmuir or linear isotherm model. Dashes denote parameters with very large error estimates due to the poor fit of the specified model. Numbers in parenthesis give the standard error of the mean.

Table 2.2 summarizes the fitted isotherm model parameters. The $q_{m, s}$ and $c_{p, m}$ values for the polymer-grafted systems show that the protein binds primarily to the surface, due to the greater number of multivalent interactions that can be made there. A surface-bound protein molecule interacts with approximately 5 surface ligands on average, providing a very favorable enthalpy which outweighs the entropic penalty of losing translational degrees of freedom upon binding to the surface. A molecule associated with the charged polymers retains its translational motion but interacts with only 1-2 ligands, making partitioning into this part of the pore less favorable. Finally, we note that although the near rectangularity of the $q_{s}$ versus $c_{p}$ isotherm results in significant uncertainty in the initial slope (and thus $K_{s}$ ), the transport rates predicted from numerical simulation 
based on the parameters given in Table 2.2 are virtually unaffected by changes in $K_{s}$ in this range.

\subsection{Molecular diffusivities}

MD simulations show that the diffusional mobility of a protein molecule depends on its location within the adsorbent and, if the molecule is adsorbed, on the strength of electrostatics. Fig. 2.9a compares the ensemble-averaged MSD curves for protein within the pore space of the macroporous system, within the polymerfilled pore space, and adsorbed on the surface in the macroporous system, for $\epsilon_{r}=10$ at which electrostatic interactions are strong. As shown, molecules not bound to the surface exhibit random walk behavior even at short time scales. In this case, individual $D$ measurements are estimated from the slope of the curve between $t=5$ and $10 \mathrm{~ns}$, using a 3D MSD calculated from a $60 \mathrm{~ns}$ section of the trajectory. Molecules bound to the surface exhibit anomalous subdiffusion over short time scales and true random walk behavior only at longer times. In this case, individual $D$ measurements are estimated from the slope of the curve between $t=30$ and $60 \mathrm{~ns}$, using an 2D MSD calculated from a $120 \mathrm{~ns}$ section of the trajectory. These times are within the linear regions of the respective curves and avoid poor sampling at long times.

Fig. $2.9 \mathrm{~b}$ compares the ensemble-averaged diffusivities of protein in the pore space, $D_{p}$, and on the surface, $D_{s}$, for both adsorbents and different $\epsilon_{r}$ values. In the macroporous system, $D_{p}$ is consistent with the experimental free solution diffusivity of $D_{0}=1.2 \times 10^{-6} \mathrm{~cm}^{2} / \mathrm{s}$ (shown in the dotted line) and does not vary with $\epsilon_{r}$, within error, as protein molecules in the open pore space do not interact with any ligands. In the polymer-grafted system, $D_{p}$ is within $30-40 \%$ 
of $D_{0}$. The effect of the charged polymers on the protein's diffusional mobility becomes more significant at $\epsilon_{r}=10$. With $\epsilon_{r}=8$ in a comparable polymer-grafted system (not shown), $D_{p}$ is reduced to approximately $50 \%$ of $D_{0}$. $D_{p}$ within the polymer-filled pore is independent of the local protein concentration in the pore space, as shown in Table 2.3, suggesting that protein-protein interactions do not impact the mobility under these conditions. 

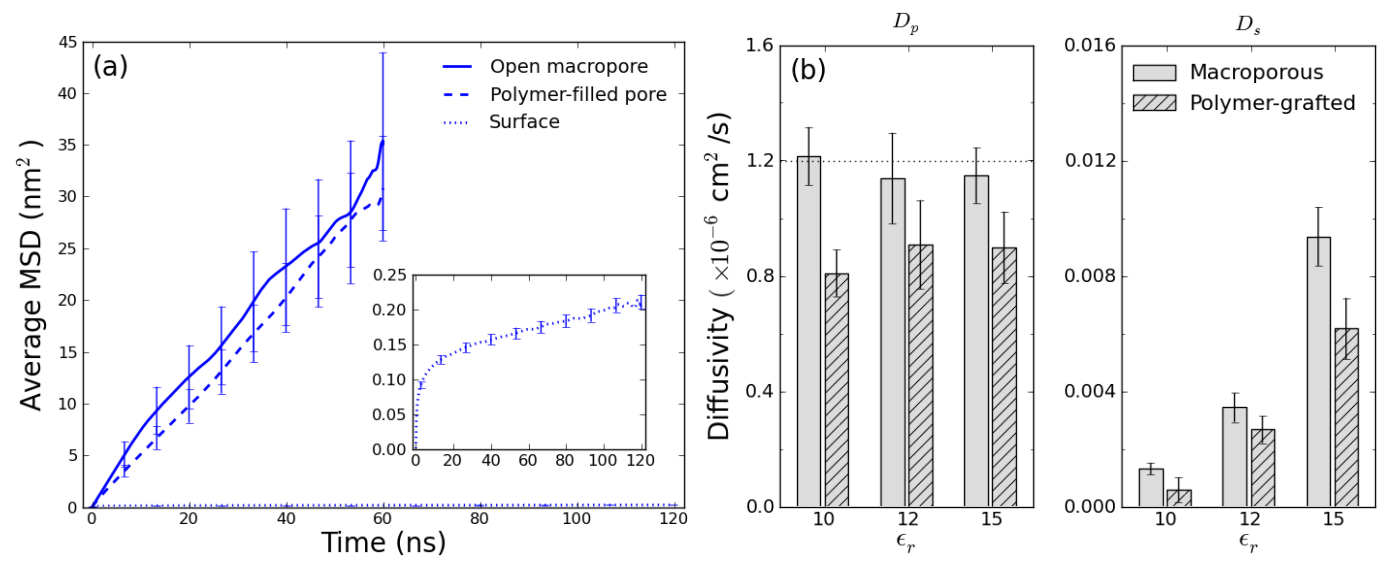

Figure 2.9: MD simulations show that the molecular diffusion of lysozyme depends on the location of the protein within the pore and on the strength of electrostatics. (a) Average MSDs of protein molecules in different phases within a pore, for favorable binding conditions represented by $\epsilon_{r}=10$. (b) Average diffusivity of protein in the pore space, $D_{p}$, and bound to the surface, $D_{s}$, for both adsorbent types and different $\epsilon_{r}$ values. The experimental free solution diffusivity of lysozyme, $D_{0}$, is shown by the dotted line alongside the $D_{p}$ results. Diffusion in the polymer-filled pore is up to $30 \%$ slower than in free solution, while diffusion on the surface is up to 3 orders of magnitude slower than $D_{0}$. Error bars show the standard error of the mean MSDs (a) and diffusivities (b).

\begin{tabular}{|ll|ll|}
\hline & \multicolumn{2}{|c|}{$\epsilon_{r}=8$} & \multicolumn{2}{c|}{$\epsilon_{r}=10$} \\
\hline$c_{p}\left(\mathrm{mg} / \mathrm{cm}^{3}\right)$ & $D_{p}\left(\times 10^{-5} \mathrm{~cm}^{2} / \mathrm{s}\right)$ & $c_{p}\left(\mathrm{mg} / \mathrm{cm}^{3}\right)$ & $D_{p}\left(\times 10^{-5} \mathrm{~cm}^{2} / \mathrm{s}\right)$ \\
\hline $15.4(0.8)$ & $0.082(0.003)$ & $15.3(0.5)$ & $0.094(0.003)$ \\
$38.3(0.6)$ & $0.082(0.005)$ & $22.4(0.4)$ & $0.091(0.004)$ \\
\hline
\end{tabular}

Table 2.3: Average diffusivity of protein molecules in the polymer-filled pore and not bound to the surface, $D_{p}$, for systems with different pore concentration, $c_{p}$. Each data point is measured from a MD simulation with a different protein loading $N$. Diffusion is independent of the protein concentration, suggesting that the polymer phase is relatively dilute.

The observed $D_{s}$ values are lower than $D_{0}$ by up to three orders of magni- 
tude as a result of strong binding to the surface. In general, $D_{s}$ increases as a function of $\epsilon_{r}$, as the weaker adsorption when electrostatics are significantly screened enhances the mobility on the surface. The lower $D_{s}$ values observed in the polymer-grafted system are attributed to the additional diffusional hindrance of the polymers grafted to the surface.

\subsubsection{Adsorption kinetics}

Numerical simulations based on the MD results given above are used to study how the strength of electrostatics affects the macroscopic adsorption kinetics. Fig. 2.10a shows simulated intraparticle profiles of the total protein concentration in the adsorbent, $\hat{q}$, for the macroporous (filled symbols) and polymer-grafted (open symbols) systems for different $\epsilon_{r}$. Under favorable binding conditions the polymer-grafted system exhibits faster adsorption kinetics than the macroporous, as seen in the fronts that evolve faster for $\epsilon_{r}=10$ and 12. No enhancement is observed for $\epsilon_{r}=15$. Both materials exhibit a sharp adsorption front at $\epsilon_{r}=10$ and a more diffuse front as $\epsilon_{r}$ increases. This is due to the fact that surface adsorption becomes less favorable as electrostatics are more significantly screened. The shape of these curves compare qualitatively with infrared microscopy images of lysozyme adsorption in macroporous and dextran-grafted particles. [20] 

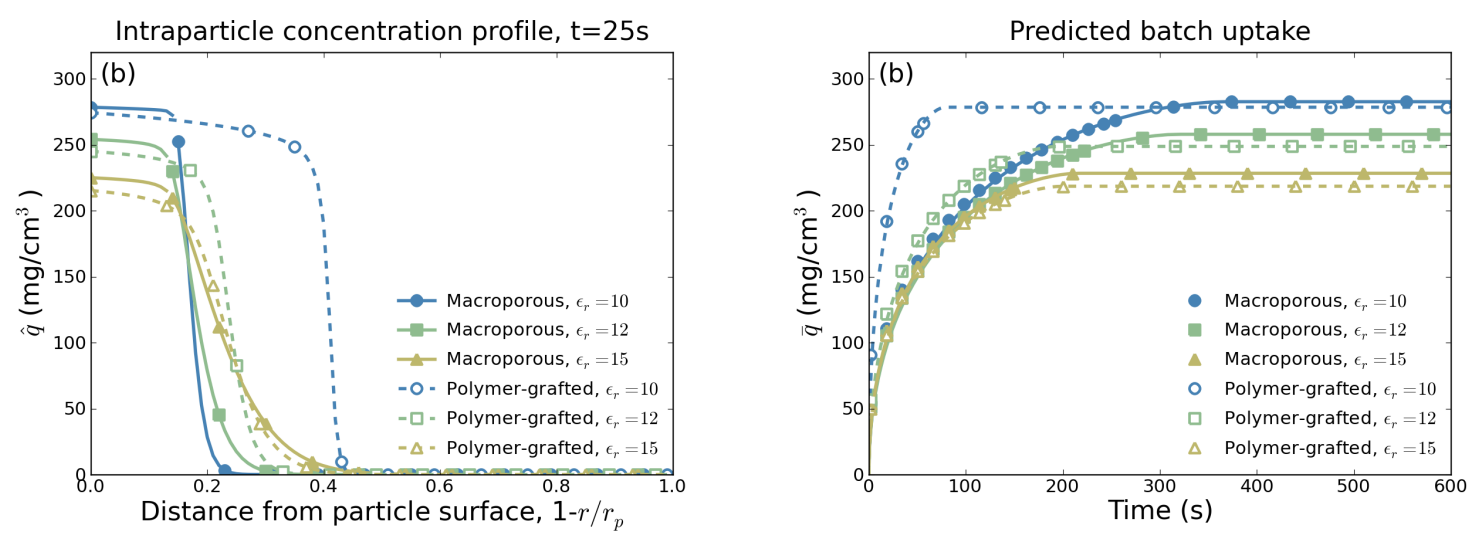

Figure 2.10: Mass transfer simulations show that when electrostatics are strong at $\epsilon_{r}=10$, the polymer-grafted system provides faster adsorption kinetics than the macroporous, and when electrostatics are weak at $\epsilon_{r}=15$, the two adsorbents exhibit comparable kinetics, as shown in (a) intraparticle profiles of the total protein concentration, $\hat{q}$, at $t=25 \mathrm{~s}$ and (b) batch uptake curves of the particleaveraged concentration, $\bar{q}$, versus $t$.

Fig. 2.10b plots batch uptake curves showing the particle-averaged concentration, $\bar{q}$, versus time. The faster kinetics of the polymer-grafted system at low $\epsilon_{r}$ are evident in the shorter times required to reach saturation. In general, the time scales over which both adsorbents are predicted to saturate are comparable to those observed for the experimental systems, shown in Fig. 1 of Ref. 7.

Fig. 2.11a shows $D_{e, a p p} / D_{0}$ as a function of $\epsilon_{r}$ for both systems, determined by fitting the batch uptake curves of Fig. 2.10b. For comparison, Fig. 2.11b provides experimental $D_{e, \text { app }} / D_{0}$ values for lysozyme in physical macroporous and dextrangrafted systems at different ionic strengths, originally presented in Fig. 2 of Ref. 7, and obtained using the same fitting procedure on the experimental batch uptake curves. Uncertainties in the simulated $D_{e, a p p} / D_{0}$ values are obtained by a 
bootstrap procedure with 50 bootstrap repetitions. For each repetition, $D_{e, a p p} / D_{0}$ is estimated using randomly selected isotherm parameters and diffusivities from the distributions of bootstraps samples, generated as discussed in the previous sections. The uncertainty in $D_{e, a p p} / D_{0}$ is estimated as the standard deviation of these $50 D_{e, a p p} / D_{0}$ values. 

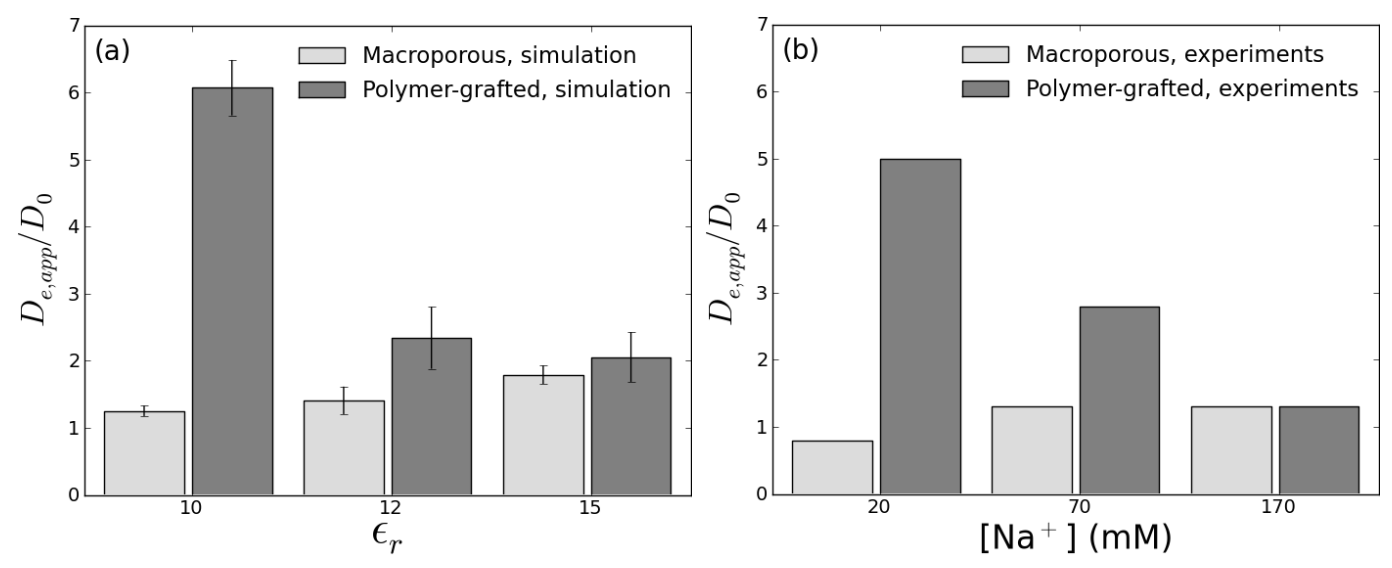

Figure 2.11: Multiscale simulation results (a) show that the effective transport rate of lysozyme, as measured by $D_{e, a p p} / D_{0}$, is enhanced in a polymer-grafted adsorbent under favorable binding conditions, which agrees qualitatively with previous experimental results (b) originally reported in Ref. 7. Error bars show the standard error of the mean.

The trends of the simulations and experiments are in qualitative agreement. Both show that $D_{e, a p p} / D_{0}$ is enhanced in the polymer-grafted system when electrostatics are strong, i.e. low $\epsilon_{r}$ in the simulations and low salt concentration in the experiments, and that the polymer-grafted transport rate approaches that of the macroporous system as electrostatics become weaker. In both cases, $D_{e, a p p} / D_{0}$ in the macroporous system increases with respect to $\epsilon_{r}$, which according to our modeling reflects the increase in surface diffusion as electrostatics become weaker.

Even for the strong-binding case of $\epsilon_{r}=10$, surface diffusion has a nonnegligible contribution to the overall transport rate, as seen in the result of $D_{e, a p p} / D_{0}>1$. The fact that the experiment shows $D_{e, a p p} / D_{0}<1$ under strong binding conditions indicates that our model surface deviates somewhat from the physical system. While trial MD simulations did not identify surface model pa- 
rameters which led to $D_{e, a p p} / D_{0} \rightarrow 1$, the numerical simulation results given in Fig. 2.6 show that this limit can be reached when surface diffusion is negligible, which would be the case with a more physically-realistic model. Because this affects both the macroporous and polymer-grafted systems, the two transport rates can still be readily compared, which in this study is of greater importance that modeling the fine details of the pore surface with high accuracy.

\subsection{Discussion}

The multiscale simulations show that in a polymer-grafted adsorbent, the protein transport rate is accelerated when partitioning from the bulk into the polymerfilled pore space is very favorable, as illustrated in Fig. 2.7. Under these conditions, the increase in $c_{p}$ more than offsets the decrease in protein diffusion due to interactions with the charged polymers, leading to a large $\partial c_{p} / \partial r$ driving force for intraparticle transport. When binding conditions are less favorable, the transport rate is not enhanced in the polymer-grafted system because the increase in $c_{p}$ is insufficient to affect the overall mass transfer flux. The relationship between partitioning into the polymer-filled pore space and the transport rate could have implications for material design. Protein affinity for the charged polymers could be enhanced by either increasing the extent to which each graft is functionalized, or by grafting a higher density of polymers to the surface. However, an overly high graft density could negatively affect the total binding capacity if it significantly reduced protein adsorption to the pore surface, as is observed in our MD simulations. Greater flexibility of the polymer grafts might also increase the number of multivalent interactions that protein molecules can make with the polymers, and as a result, the amount of partitioning into the pore space. 
In some systems, protein affinity for the polymer grafts and/or the underlying surface could also be affected by dispersion interactions or water-mediated hydrophobic interactions. By neglecting these in our simulations, we assume that the neutral protein residues and dextran monomers have approximately the same affinity for water as for each other, and that protein affinity for the adsorbent is dominated by the electrostatics of the overall net charge. The extent to which this approach can capture experimental trends when applied to different types of proteins will inform future modifications to the force field.

The geometry of the adsorbent pore could also affect the adsorption and diffusion of certain proteins. For large proteins, the curvature of the pore surface over long length scales may affect the number of molecules that can adsorb to the surface, just as the surface roughness on short length scales affects lysozyme's adsorption. The surface geometry can also affect the structure of the grafted polymer phase as has been shown in a previous MD study, [59] which would likely impact the protein's diffusion through the polymers and accessibility to the surface. These effects may not be significant for lysozyme due to its relatively small size, however, various pore geometries should be considered in MD simulations of larger proteins.

Simulations of other proteins will also be necessary to determine if our model can predict the correct shape of the intraparticle concentration profile in a given adsorbent material. Confocal laser scanning microscopy (CLSM) images for different proteins and adsorbents have shown qualitatively different profiles for certain proteins in open-pore and polymer-grafted ion exchange particles under transient adsorption conditions, suggesting that transport occurs via different diffusion mechanisms. [22, 60] Sharp adsorbed protein concentration profiles have been observed for strong binding conditions in open-pore materials, indicating that 
surface diffusion plays little or no role, as that mechanism would lead to more diffuse adsorption profiles. On the other hand, diffuse profiles have been obtained for some proteins in polymer-grafted materials, indicating that adsorbed protein molecules remain mobile. These differences have not been observed for lysozyme in light microscopy experiments, [9] nor are they observed in the simulation results presented here. However, preliminary numerical simulations (not shown) do predict more diffuse profiles for lysozyme when a greater percentage of the protein within the pore is associated with the charged polymers, where diffusion is much faster than on the surface.

\subsection{Conclusions}

A multiscale modeling approach is developed to estimate the adsorption capacity and transport rate of protein in IEC media with different pore architectures, based on the molecular-level details of the system. Coarse-grained MD simulations are used to determine the partitioning and diffusion of protein molecules within the adsorbent pore, and based on these molecular behaviors, numerical simulation of mass transfer over longer scales is used to quantify the effective transport rate.

A series of numerical simulations were initially performed to predict how different levels of protein diffusion and partitioning on the surface and in the polymers affect the effective transport rate in macroporous and polymer-grafted adsorbents. Based on these results, we determined molecular model parameters for the agarose surface, the charge content per grafted polymer, and the strength of electrostatics under favorable binding conditions that lead to simulated transport rates that agree with previous experiments. These model parameters were used to study how varying the strength of electrostatic affects adsorption behav- 
iors.

Consistent with previous experiments, we observe that the binding capacities of both adsorbents and the enhancement in the transport rate of the polymergrafted adsorbent decrease as electrostatic interactions become weaker. The enhanced transport in the polymer-grafted system when electrostatics are strong is attributed to favorable protein partitioning into the polymer-filled pore space, and the fact that the protein associated with the charged polymers retains significant diffusional mobility. Whereas adsorption to the surface decreases the protein's diffusivity by up to three orders of magnitude relative to $D_{0}$, association with the charged polymers leads to only a $40 \%$ reduction when electrostatics are strong. The simulations show that the macroporous and polymer-grafted adsorbents have similar overall binding capacities under favorable binding conditions, in contrast with experiments showing a 10-20\% higher binding capacity for lysozyme in a dextran-grafted adsorbent versus a macroporous material. As discussed in the subsequent chapters, this modeling approach can be extended in a straightforward manner to proteins and adsorbents with different characteristics in order to better understand how molecular details influence macroscopic protein adsorption behaviors, and to utilize this understanding to help design efficient separation processes in the future. 


\section{Chapter 3}

\section{Effects of Polymer Graft Properties}

\subsection{Introduction}

In this chapter, multiscale modeling is used to study the adsorption of lysozyme onto ion-exchangers obtained by grafting charged polymers into a porous matrix, in systems with various polymer properties and strengths of electrostatic interaction. Molecular dynamics simulations show that protein partitioning into the polymer-filled pore space increases with the overall charge content of the polymers, while the diffusivity in the pore space decreases. However, the combination of greatly increased partitioning and modestly decreased diffusion results in macroscopic transport rates that increase as a function of charge content, as the large concentration driving force due to enhanced pore space partitioning outweighs the reduction in the pore space diffusivity. Matrices having greater charge associated with the grafted polymers also exhibit more diffuse intraparticle concentration profiles during transient adsorption. In systems with a high charge content per polymer and a low protein loading, the polymers preferentially partition towards the surface due to favorable interactions with the surface-bound 
protein.

\subsection{Background}

Various studies have shown that protein adsorption in polymer-grafted materials can depend significantly on the polymer graft density, the charge content of the polymers, and the salt concentration. In another study, Bowes and Lenhoff showed that increasing the overall charge content in a dextran-grafted adsorbent increased the binding capacity and affinity of lysozyme, but also led to longer particle saturation times. [23] Increasing the dextran graft density for a constant charge content did not lead to clear trends in these properties for lysozyme. Yu et al. studied the adsorption of bovine serum albumin on various anion exchangers in which poly(ethylenimine) was incorporated into Sepharose Fast Flow particles at different graft densities to achieve different overall charge densities. [24] They found that a critical charge density existed at which both the binding capacity and effective diffusivity increased significantly. For the same type of resin, $\gamma$-globulin exhibited a maximum effective diffusivity at an intermediate charge density. [61] These results suggest that protein properties such as size and charge also affect the extent to which the polymer grafts enhance adsorption, as discussed in the next chapter.

Previous molecular modeling studies have provided insights into how protein diffusion and partitioning can vary with respect to the properties of polymergrafted IEC resins. Riccardi and co-workers used MD simulations that include both atomistic and coarse-grained (CG) models with an explicit solvent to study the transport of a biomolecule through a matrix of charged dextran grafts. Among their findings were that lower dextran graft densities led to larger polymer layer 
pore sizes and a relatively small gradient of local nonelectroneutrality due to the distribution of the charged ligands, both of which could affect transport through the polymers. [44] Johansson and Van Alstine studied similar systems with Monte Carlo (MC) simulations based on highly coarse-grained models. [43] Their simulations showed that protein adsorption onto charged polymer grafts was dependent on the salt concentration and protein surface charge density, but was relatively independent of the polymer graft density.

These studies have suggested mechanisms by which the polymer graft properties can affect an individual adsorbate's partitioning and transport. However, as demonstrated in Chapter 2, the simulation of larger ensembles of molecules can allow for qualitative prediction of macroscopic adsorption properties. Connecting a system's molecular characteristics to these practical quantities would be particularly useful for understanding how a polymer-grafted resin may be modified to enhance the adsorption of a particular protein.

Therefore, this chapter discusses a systematic study on how the properties of charged polymer grafts and the bulk solution affect a protein's adsorption and transport on both the molecular and macroscopic levels. The model IEC system is comprised of dextran polymers functionalized with sulfonate groups and grafted to a macroporous agarose matrix, with lysozyme as the protein. We extend the modeling approach introduced in Chapter 2 to systems with different polymer charge content, graft density, and strength of electrostatics to predict how these variables qualitatively affect the protein's partitioning and diffusion within the pore, its effective transport rate, and the structure of the grafted polymers. 


\subsection{Molecular simulation details}

MD simulations of an ensemble of lysozyme protein molecules within a IEC pore containing charged dextran grafts are used to determine protein partitioning and diffusion in different regions of the pore. Figures $4.1 \mathrm{a}$ and $3.1 \mathrm{~b}$ show example snapshots from simulations of adsorbents with different polymer graft densities, and protein loadings that correspond to near-saturation conditions at equilibrium.
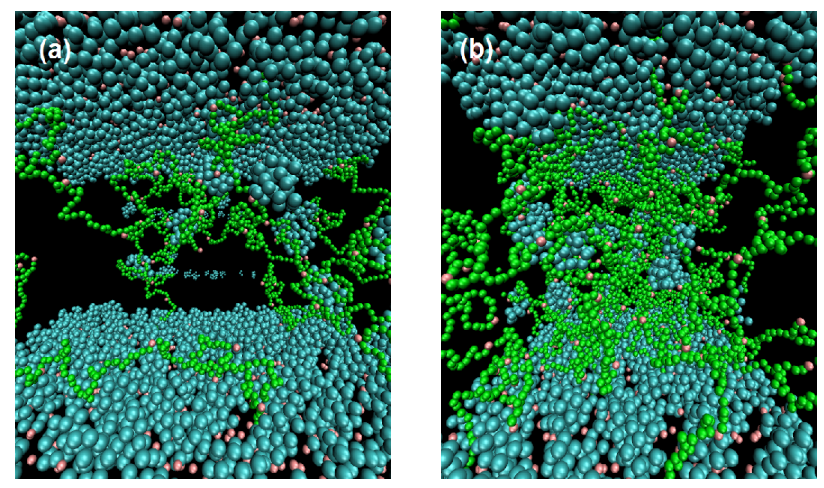

Figure 3.1: Snapshots from equilibrated MD simulations of lysozyme within an adsorbent pore. The dextran graft density corresponds to the estimated graft density of the experimental material, $\sigma_{e}$, in (a), and $3 \sigma_{e}$ in (b). Both adsorbents are near saturation. Lysozyme, dextran, and sulfonate ligands (attached both to the surface and to the dextran) are shown in blue, green, and pink, respectively.

The details of the MD simulations used in this study are the mostly the same as those described in Chapter 2. Nine different adsorbents are tested in this study, with values of the per-polymer charge content, $f_{P L}$, of $0.05,0.1$, and 0.2 , and values of the graft density, $\sigma$, of 1,2, and 3 times the estimated graft density of the experimental material, $\sigma_{e}$. [9] $f_{P L}=0.05$ is believed to be most consistent with the experimental material, assuming that the dextran chains have approximately $5 \%$ side chain branching [57] and that each side chain branch is functionalized. [9] 
Thus, the system with $f_{P L}=0.05$ and $\sigma=\sigma_{e}$ is most consistent with previous experiments and is used as our reference case. The concentration of polymerbound ligand, $c_{P L}$, ranges from $4 \times 10^{-6} \mathrm{~mol} / \mathrm{ml}$ for the reference case to 51 $\times 10^{-6} \mathrm{~mol} / \mathrm{ml}$ for the most highly charged system with $f_{P L}=0.2$ and $\sigma=3 \sigma_{e}$. The concentration of surface-bound ligands is approximately $58 \times 10^{-6} \mathrm{~mol} / \mathrm{ml}$ for all systems. Lateral dimensions of the pore space and number of grafted polymers per surface are varied to achieve the desired graft density, as described in Appendix A. All MD simulations were performed using GROMACS version 4.6.4. [55]

\subsection{Results}

\subsubsection{Adsorption Equilibria}

A series of MD simulations with different protein loadings are used to quantify the adsorption isotherms for a given system with a particular polymer charge content, graft density, and strength of electrostatics. The concentrations of protein on the surface, $q_{s}$, in the polymer-filled pore space, $c_{p}$, and in free solution, $C$, are calculated as described in Chapter 2.

Figure 3.2 shows predicted adsorption isotherms for representative systems, which demonstrate that increasing the charge content per polymer, the polymer graft density, and the strength of electrostatics all lead to greater protein partitioning into the pore space and higher overall capacities. The plots in the top row show the isotherms most often measured experimentally, in which the total adsorbed concentration, $\hat{q}$, is given as a function of free solution concentration, $C$. The plots in the middle row show the local isotherms $q_{s}$ versus $c_{p}$, which describe partitioning of protein between the polymer-filled pore space and the 
charged surface. The plots in the bottom row show $c_{p}$ versus $C$ isotherms describing partitioning between the free solution and the pore space. Curves fitted according to Eq. 2.8, Eq. 2.9, and to the sum of these models are shown alongside the values predicted from MD simulation in the plots of the middle, bottom, and top rows, respectively, of Figure 3.2. Modeling the overall isotherm as the sum of the two local equilibrium relationships allows us to capture the increase in the adsorbed concentration at high solution concentrations due to the polymer grafts (discussed below). This effect could not be captured with a single Langmuir model, which describes a single saturation regime.

The constancy of the $q_{s}$ versus $c_{p}$ isotherms shown in Figures 2e-2h (middle row) demonstrates that surface adsorption is fairly independent of the adsorbent properties. Therefore, differences in the overall capacities are primarily due to different levels of protein partitioning from solution into the polymer-filled pore space, seen in the $c_{p}$ versus $C$ isotherms shown in Figures 2i-2l (bottom row).

As seen in Figures 2i-2j, as $f_{P L}$ is increased at a constant graft density of $\sigma=\sigma_{e}$, pore space partitioning becomes increasingly favorable. The effect is most significant when electrostatic interactions are strong $\left(\epsilon_{r}=10\right)$, but even when electrostatics are weaker $\left(\epsilon_{r}=15\right)$, increasing the per-polymer charge content to $f_{P L}=0.2$ leads to enhanced pore space partitioning. As seen in Figures $2 \mathrm{k}-2 \mathrm{l}$, increasing the polymer graft density at a constant per-polymer charge content of $f_{P L}=0.05$ leads to a moderate increases in $c_{p}$ (and as a result, $\hat{q}$ ) at saturation when electrostatics are strong, but no enhancement when electrostatics are weaker.

Figure 3.3 shows the predicted saturation capacities of the entire pore, $\hat{q}^{\max }$, the surface, $q_{s}^{\max }$, and the pore space, $c_{p}^{\max }$, for all systems tested with both $\epsilon_{r}=10$ and $\epsilon_{r}=15 . c_{p}^{\max }$ is evaluated from Eq. 2.9 at $C=2 \mathrm{mg} / \mathrm{ml}, q_{s}^{\max }$ is found from 
Eq. 2.8 at $c_{p}=c_{p}^{\max }$, and $\hat{q}^{\max }=q_{s}^{\max }+c_{p}^{\max }$. The statistical uncertainty in a given saturation capacity is estimated by a similar bootstrapping procedure as described in Chapter 2, in which for each of 1000 bootstrap repetitions, we construct a local isotherm by sampling from the time series of the concentrations, fit the resulting isotherm with the appropriate isotherm model, evaluate the saturation capacity, and calculate the standard deviation of the 1000 predicted saturation capacities to estimate the standard error in the mean saturation capacity. These estimated uncertainties are low compared to the mean values, as seen in the magnitude of the error bars plotted in the center of the bars. 

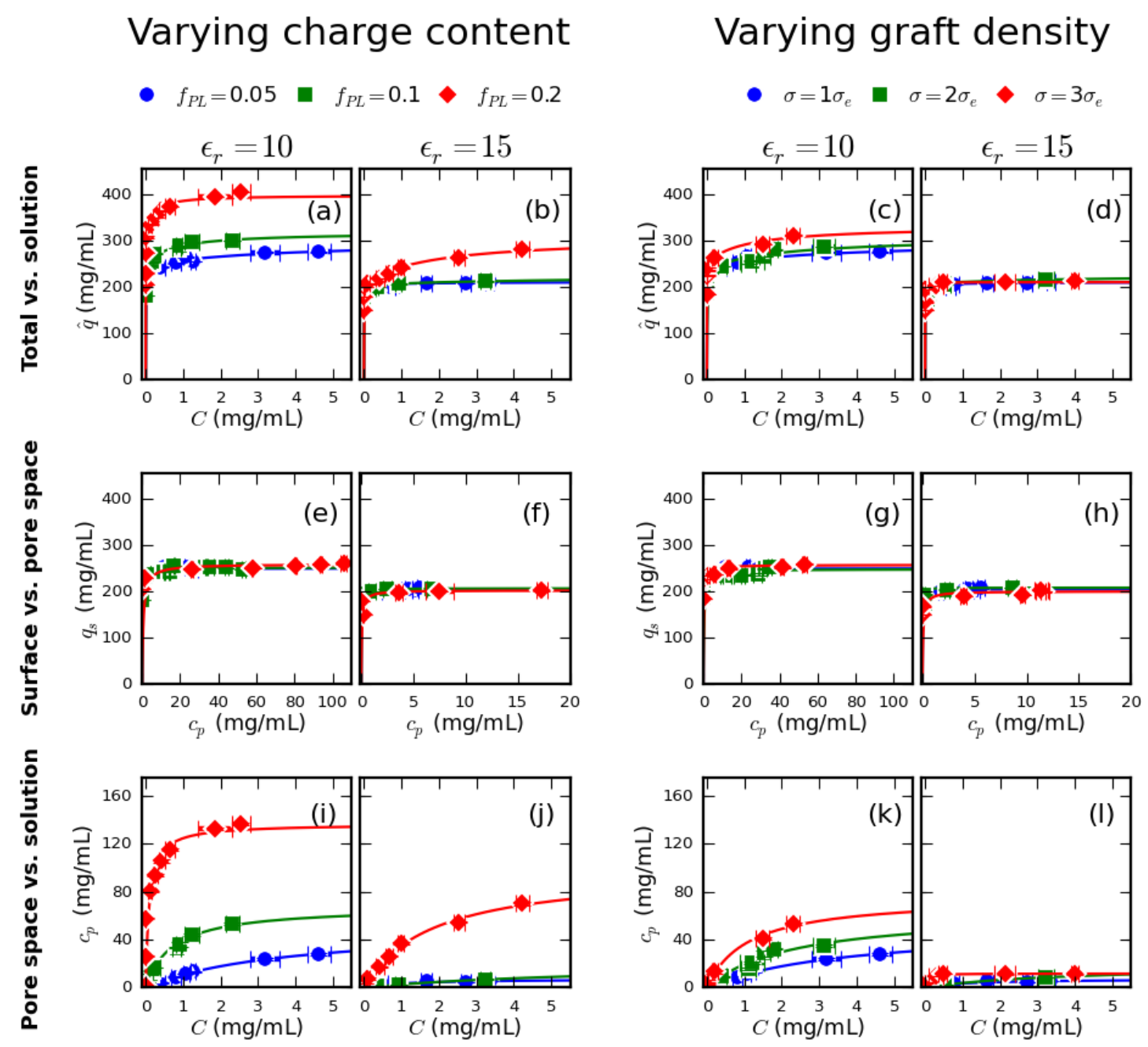

Figure 3.2: MD simulations of lysozyme in various polymer-grafted adsorbents show that increasing the charge content per polymer, $f_{P L}$, and the graft density of charged polymers, $\sigma$, can enhance lysozyme's overall adsorption capacity when electrostatics are strong, due to increased protein partitioning into the pore space. Increasing $f_{P L}$ for a constant $\sigma=\sigma_{e}$ (left columns) leads to greater partitioning into the pore space (bottom row) and a higher overall capacity (top row), particularly for $\epsilon_{r}=10$. Increasing $\sigma$ for a constant $f_{P L}=0.05$ (right columns) has a more moderate effect on the pore space partitioning and the overall capacity when $\epsilon_{r}=10$, and no effect for $\epsilon_{r}=15$. Surface adsorption is generally independent of the polymer properties tested (middle row). 
As shown in Figure 3.3a, extremely high overall capacities of over 400 and 500 $\mathrm{mg} / \mathrm{ml}$ are predicted in systems with high $f_{P L}$ and $\sigma$ values when electrostatics are strong. We note that while these calculations are based on an assumed porosity of $\epsilon_{p}=0.9$, the protein-accessible porosity may be substantially smaller in actual materials. This could lead to an over-prediction of the adsorption capacities by the model; however, in this study qualitative differences between the systems are of more interest than quantitative predictions. Again, the enhancements in the overall capacity relative to the reference case of $f_{P L}=0.05$ and $\sigma=\sigma_{e}$ are due to the enhanced pore space capacities, which in some cases approach and even exceed the capacity of the surface despite the lower concentration of ligands attached to the polymers versus the surface.

Partitioning of lysozyme into the pore space appears to be favored in adsorbents with more highly charged polymers and lower graft densities, as seen when comparing the pore space capacities of different systems with similar charge contents. To illustrate this effect, Figure 3.4 shows $c_{p}^{\max }$ versus the concentration of polymer-bound ligand, $c_{P L}$, for all systems. Both concentrations are given on a molar basis, and statistical uncertainties are smaller than the sizes of the plot symbols. When electrostatics are strong $\left(\epsilon_{r}=10\right), c_{p}^{\max }$ increases nearly linearly with $c_{P L}$. However, comparing the two systems with $c_{P L} \approx 9 \times 10^{-6} \mathrm{~mol} / \mathrm{ml}$ and the two systems with $c_{P L} \approx 17 \times 10^{-6} \mathrm{~mol} / \mathrm{ml}$, higher $c_{p}^{\text {max }}$ values (and thus higher $\hat{q}^{\max }$ values) are observed in the systems with higher per-polymer charge content and lower graft density. When electrostatics are weaker $\left(\epsilon_{r}=15\right)$, pore space partitioning is only enhanced significantly in systems with a per-polymer charge content of $f_{P L}=0.2$. At lower $f_{P L}$ values, increasing the graft density provides no increase in the overall adsorption, despite the associated increase in the pore's overall charge content. 

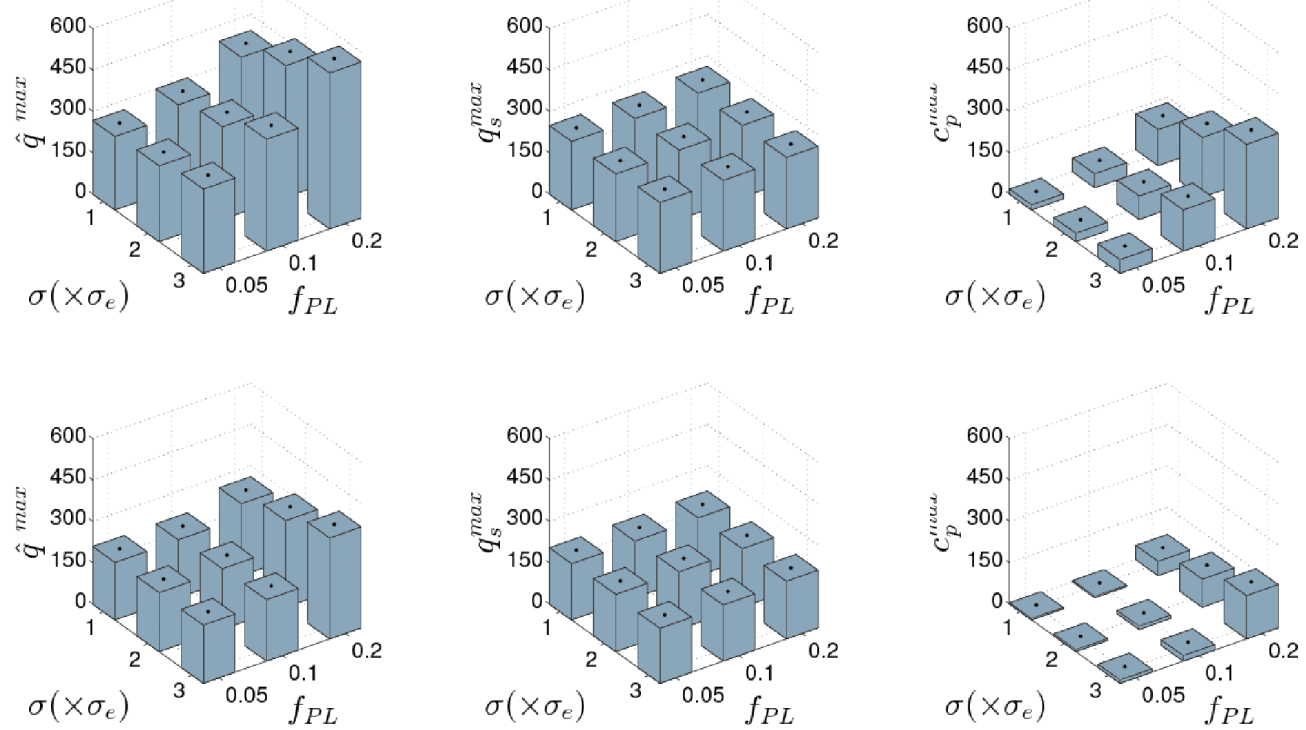

Figure 3.3: MD simulations of lysozyme in polymer-grafted adsorbents predict that more highly charged adsorbents lead to higher total saturation capacities (a and d) due to greater partitioning of protein into the pore space (c and f) when electrostatics are strong $(\mathrm{a}-\mathrm{c})$, and have less effect on adsorption capacities when electrostatics are weaker (d-f). All concentrations shown have units of $\mathrm{mg} / \mathrm{ml}$.

The adsorption equilibria results predicted by MD are qualitatively consistent with several experimental trends. The prediction that, for a given overall charge content and under favorable binding conditions, increasing $f_{P L}$ leads to a higher $c_{p}^{\max }$ than increasing $\sigma$ agrees with adsorption experiments for lysozyme in matrices with different charge contents and dextran graft densities. [23] The simulations also show that the protein is distributed throughout the entire polymer-filled pore space, in agreement with experimental observations of multilayer binding in polymer-grafted systems. [10,12] As expected for IEC systems, the simulations predict reduced levels of adsorption in all systems when electrostatics are weaker with $\epsilon_{r}=15$. Additionally, the prediction that increasing the graft density pro- 
vides no additional lysozyme adsorption when $\epsilon_{r}=15$ is likely related to the experimental observation that larger proteins can be partially excluded from the pore space by grafted polymers under high salt conditions. [10]

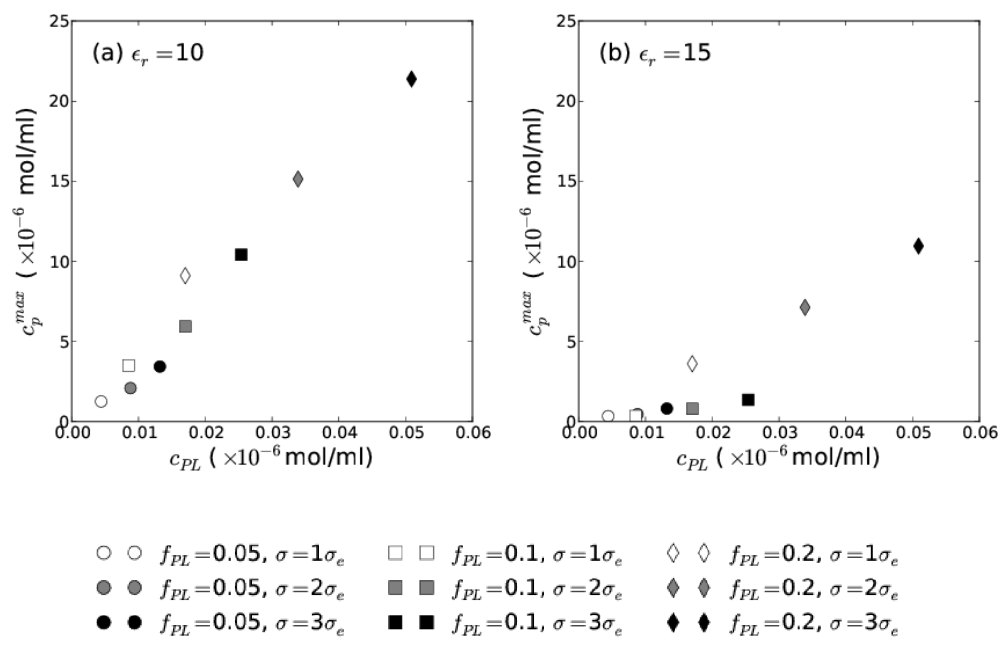

Figure 3.4: MD simulations of lysozyme in polymer-grafted adsorbents predict that the pore space saturation capacity, $c_{p}^{\max }$, varies approximately linearly with the polymer ligand concentration, $c_{P L}$, when electrostatics are strong $\left(\epsilon_{r}=10\right)$. $c_{p}^{\max }$ is less dependent on $c_{P L}$ when electrostatics are weaker $\left(\epsilon_{r}=15\right)$, except when the charge content per polymer is high.

\subsubsection{Molecular diffusivities}

Average molecular diffusivities of the protein are determined by the same approach described in Chapter 2, using the Einstein relation to calculate diffusivities of individual molecules during a single simulation with a high protein loading that corresponds to a saturated pore. As before, the surface diffusivity, $D_{s}$, is calculated from Eq. 2.4 with $N_{d i m}=2$ and the MSD slope evaluated between $\Delta t=30$ ns to $60 \mathrm{~ns}$. For each new system considered here, the pore space diffusivity, $D_{p}$, 
is calculated with $N_{\text {dim }}=3$ and the MSD slope also evaluated between $\Delta t=30$ $\mathrm{ns}$ to $60 \mathrm{~ns}$, as protein in these systems experienced more hindered diffusion in the pore space than in the reference case considered in Chapter 2.

Figure 3.5 shows the average pore space diffusivities, $D_{p}$, predicted from the MD simulations as a function of $c_{P L}$. The results suggest that diffusion can be significantly hindered when the polymer ligand concentration is high, depending on the strength of electrostatics. When binding conditions are favorable $\left(\epsilon_{r}=10\right)$, $D_{p}$ is approximately inversely related to the charge content per polymer, $f_{P L}$, for each tested graft density $\sigma$. The dependence on graft density (for a given perpolymer charge content) is less clear, though diffusion generally is most hindered in the systems with $\sigma=3 \sigma_{e}$. The variation in $D_{p}$ with respect to $c_{P L}$ reflects the increased prevalence of multivalent interactions between protein and the polymer at higher polymer ligand interactions, as these multivalent interactions hinder mobility to a greater extent. At very high $c_{P L}$, protein-protein interactions also contribute to the decrease in $D_{p}$ due to the high local protein concentrations within the polymers.

Pore space diffusivities are generally higher when $\epsilon_{r}=15$ than when $\epsilon_{r}=$ 10 due to the weaker protein-polymer interactions when electrostatics are more highly screened. For this case, $D_{p}$ appears to be controlled by the graft density for $\sigma=2 \sigma_{e}$ and $\sigma=3 \sigma_{e}$, and is independent of $f_{P L}$. Only at the lowest tested graft density of $\sigma=\sigma_{e}$ does $D_{p}$ decrease significantly with respect to $f_{P L}$. These results suggest that the degree to which diffusion is hindered by steric effects versus electrostatic attraction to the ligands depends on the screening of charged interactions.

The average predicted surface diffusivities vary from $D_{s}=0.5 \pm 0.2 \times 10^{-9}$ $\mathrm{cm}^{2} /$ sto $1.2 \pm 0.4 \times 10^{-9} \mathrm{~cm}^{2} /$ sfor $\epsilon_{r}=10$, and from $3.9 \pm 1.8 \times 10^{-9} \mathrm{~cm}^{2} /$ sto 
$7.1 \pm 2.8 \times 10^{-9} \mathrm{~cm}^{2} /$ sfor $\epsilon_{r}=15$. As discussed in Chapter 2, $D_{s}$ variations in this range have little impact on the overall transport rate for these systems, as transport is largely controlled by the partitioning and diffusion of protein within the pore space.
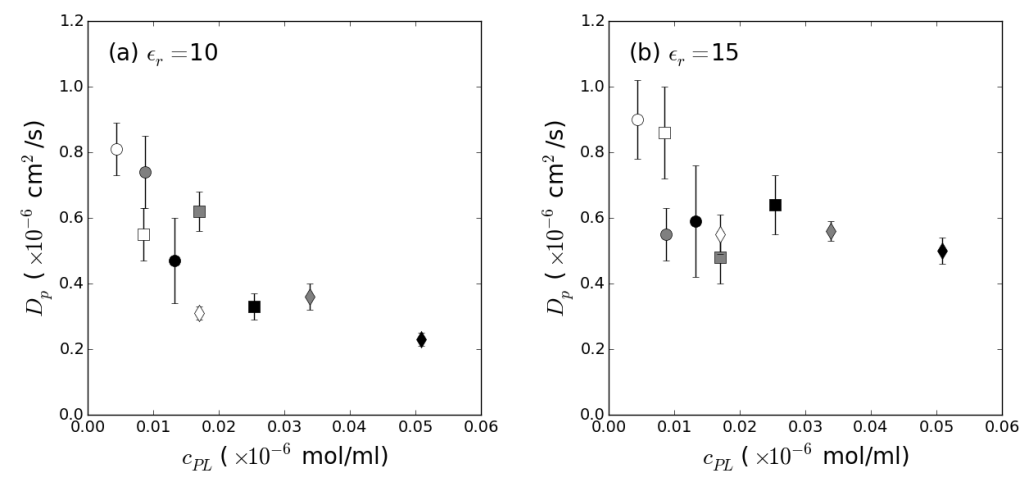

$$
\begin{aligned}
& \bigcirc f_{P L}=0.05, \sigma=1 \sigma_{e} \quad \square \square f_{P L}=0.1, \sigma=1 \sigma_{e} \quad \diamond \diamond f_{P L}=0.2, \sigma=1 \sigma_{e} \\
& \circ f_{P L}=0.05, \sigma=2 \sigma_{e} \quad \square \square f_{P L}=0.1, \sigma=2 \sigma_{e} \quad \diamond \vee f_{P L}=0.2, \sigma=2 \sigma_{e} \\
& \text { - } f_{P L}=0.05, \sigma=3 \sigma_{e} \backsim f_{P L}=0.1, \sigma=3 \sigma_{e} \bullet f_{P L}=0.2, \sigma=3 \sigma_{e}
\end{aligned}
$$

Figure 3.5: MD simulations of lysozyme in polymer-grafted adsorbents predict that the average protein diffusivity in the pore space, $D_{p}$, decreases more significantly with respect to the polymer ligand concentration, $c_{P L}$, when electrostatics are strong $\left(\epsilon_{r}=10\right)$.

\subsubsection{Adsorption kinetics}

As described in detail in Chapter 2, the effective transport rate of the protein in each adsorbent is determined from numerical simulation of mass transfer based on the molecular behaviors of the protein predicted from MD. Figure 3.6 shows the predicted $D_{e, a p p} / D_{0}$ values as a function of $c_{P L}$ for both $\epsilon_{r}=10$ and $\epsilon_{r}=$ 15. The transport rate generally increases with respect to the charge content of the adsorbent when electrostatics are strong $\left(\epsilon_{r}=10\right)$. Thus, according to the 
model, the decrease in $D_{p}$ with respect to $c_{P L}$ seen in Figure 3.4 is outweighed by the increased capacity of the polymers as their overall charge content increases, as seen in Figure 3.5. This can be understood by recognizing that $D_{e, a p p} / D_{0}$ is approximately proportional to the product of the adsorbed concentration and the diffusivity of adsorbed protein. [5] One exception to this trend of an increase in the polymer capacity outweighing a reduction in $D_{p}$ is seen comparing the system with $f_{P L}=0.1$ and $\sigma=2 \sigma_{e}$ to the system with $f_{P L}=0.2$ and $\sigma=\sigma_{e}$, which have the same overall charge content. The system with higher graft density has a $40 \%$ higher $D_{e, a p p} / D_{0}$, but in this case its relatively high $D_{p}$ outweighs its lower polymer capacity. When electrostatics are weaker $\left(\epsilon_{r}=15\right)$, systems with a high per-polymer charge content of $f_{P L}=0.2$ exhibit enhanced transport rates that increase with respect to $\sigma$, while the systems with lower $f_{P L}$ all exhibit relatively low transport rates. For the adsorbents with the highest $c_{P L}$ values, $D_{e, a p p} / D_{0}$ at $\epsilon_{r}=15$ is comparable to or higher than the $D_{e, a p p} / D_{0}$ values observed at $\epsilon_{r}=10$, as the relatively high diffusivities and low overall capacities when electrostatics are weak lead to fast saturation rates.

The predicted trends that the transport rate and overall adsorption capacity both increase with the overall polymer charge content do not match previous experimental results showing that more highly charged adsorbents lead to higher capacities but slower uptake rates. [23] One reason for this discrepancy may be that the experimental systems are in a high ligand concentration regime in which the concentration of polymer-associated protein is high enough to cause steric hindrance, whereas our simulations have lower ligand concentrations due to the material properties of the experimental materials [9] and thus lower polymerassociated protein concentrations. Additionally, no information is available on the distribution of charged ligands between grafted polymers and the underlying 
agarose surface in the actual experimental system. Differences in this distribution could affect adsorption capacities, binding strengths, and diffusion, resulting in different predicted trends. The model prediction of more highly charged polymers leading to faster transport is expected given the polymers' large positive effect on the concentration driving force and relatively small negative effect on protein diffusivities, however it is likely that greater partitioning of ligands onto the surface would would lead to slower transport rates under favorable binding conditions.
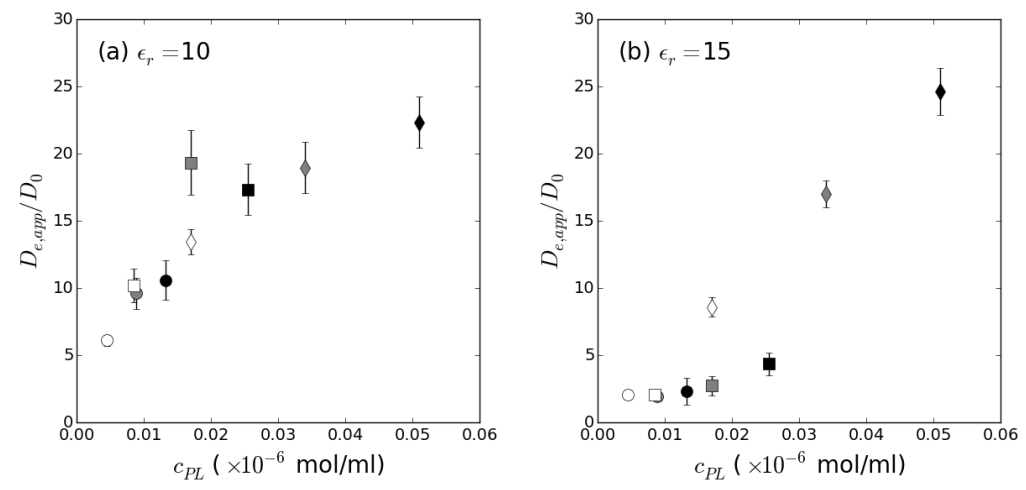

$$
\begin{aligned}
& \bigcirc f_{P L}=0.05, \sigma=1 \sigma_{e} \quad \square \square f_{P L}=0.1, \sigma=1 \sigma_{e} \quad \diamond \diamond f_{P L}=0.2, \sigma=1 \sigma_{e} \\
& \text { - } f_{P L}=0.05, \sigma=2 \sigma_{e} \square \square f_{P L}=0.1, \sigma=2 \sigma_{e} \diamond \diamond f_{P L}=0.2, \sigma=2 \sigma_{e} \\
& \text { - } f_{P L}=0.05, \sigma=3 \sigma_{e} \quad \square \quad f_{P L}=0.1, \sigma=3 \sigma_{e} \bullet f_{P L}=0.2, \sigma=3 \sigma_{e}
\end{aligned}
$$

Figure 3.6: Mass transfer simulations of lysozyme in polymer-grafted adsorbents predict that the effective transport rate, $D_{e, a p p} / D_{0}$, generally increases with respect to polymer ligand concentration, $c_{P L}$, when electrostatics are strong $\left(\epsilon_{r}=10\right)$. When electrostatics are weaker $\left(\epsilon_{r}=15\right), D_{e, a p p} / D_{0}$ increases with respect to the graft density when the charge content per polymer is high.

Figure 3.7 shows simulated intraparticle concentration profiles for the various systems, which illustrate how the charged polymer properties and strength of electrostatics can impact the pattern of adsorption into the particle. While for all 
systems, the concentration profile has a sharp front located at its maximum distance from the particle surface, the profiles for more highly charged adsorbents are relatively diffuse at shorter distances from the surface. This is most evident when electrostatics are strong $\left(\epsilon_{r}=10\right)$, and to a lesser degree with weaker electrostatics. The diffuse profiles observed in the highly charged systems appear to correlate with the higher pore space concentrations and slower pore diffusivities for these same systems, shown in Figures 3.4 and 3.5, respectively.

Diffuse intraparticle concentration profiles are typically associated with the solid diffusion mass transfer mechanism, in which the driving force is the concentration gradient of adsorbed protein. The ordinary pore diffusion mechanism leads to sharp concentration profiles and has a driving force of the concentration gradient of unbound protein in the pore space. [5] Previous confocal laser scanning microscopy experiments have shown that certain proteins exhibit sharp adsorption fronts in macroporous matrices and more diffuse fronts in particles with charged polymer grafts. [22] The profiles shown in Figure 3.7 suggest that the sharpness of the adsorption front in these polymer-grafted systems varies dependent on the relative amounts of protein bound to the underlying surface and polymers. For these systems, mass transfer may be best described by the "partial-shrinking core" model used by Bowes and Lenhoff to describe uptake in commercial dextran-grafted materials. [21]

Though beyond the scope of this study, the charge and size of the protein could also dictate the conditions under which increasing the per-polymer charge content and/or graft density enhances adsorption properties. Our simulations suggest that either type of polymer modification enhances enhances the pore space partitioning and transport rate of lysozyme, a relatively small model protein. However, it is possible that only low polymer graft densities would enhance 
the adsorption properties of a more weakly charged and/or larger protein, as steric hindrance from the neutral polymer segments may become important in these cases.
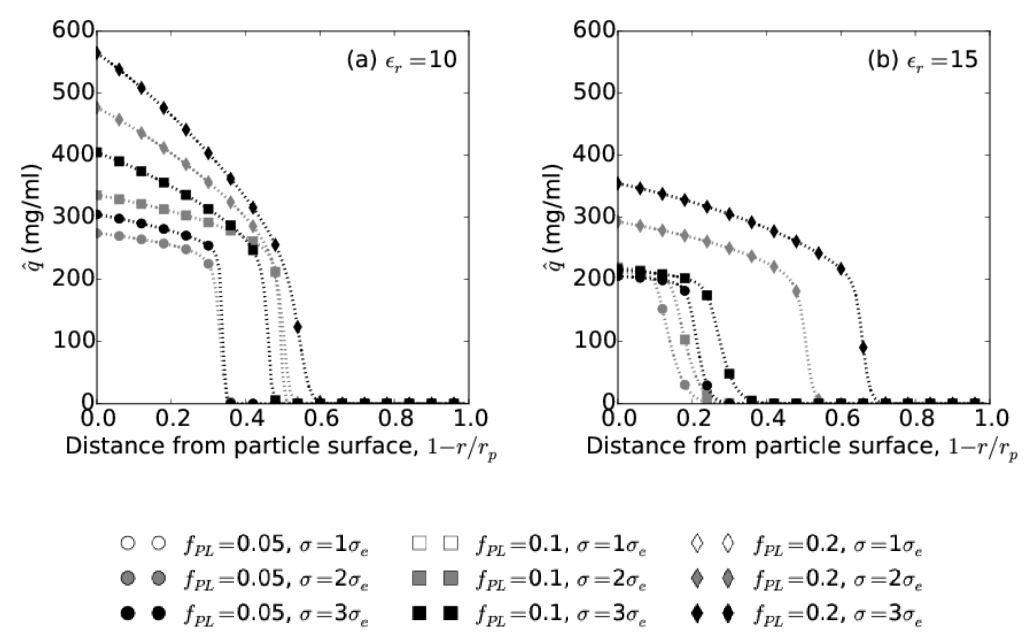

Figure 3.7: Mass transfer simulations of lysozyme in polymer-grafted adsorbents predict that the adsorption front into the particle changes qualitatively with respect to adsorbent properties and the strength of electrostatics. Intraparticle concentration profiles evaluated at $t=10 \mathrm{~s}$ are shown for all systems tested. The more highly charged adsorbents exhibit more diffuse concentration profiles, particularly when electrostatics are strong $\left(\epsilon_{r}=10\right)$.

\subsubsection{Polymer phase properties}

The MD simulations were analyzed to determine the equilibrium partitioning of the charged polymers throughout the pore space. The different adsorbents exhibit similar polymer structures under saturation conditions but very different structures at low protein loadings. This is evident in Figures 3.8a and 3.8b, which show results at high and low loadings, respectively, for the average number density of dextran monomers normalized by the pore surface area, $N_{D} / S$, with 
respect to $z$ position in the pore. Binding conditions are favorable with $\epsilon_{r}=10$. Each curve is averaged over 1 microsecond of simulation data.
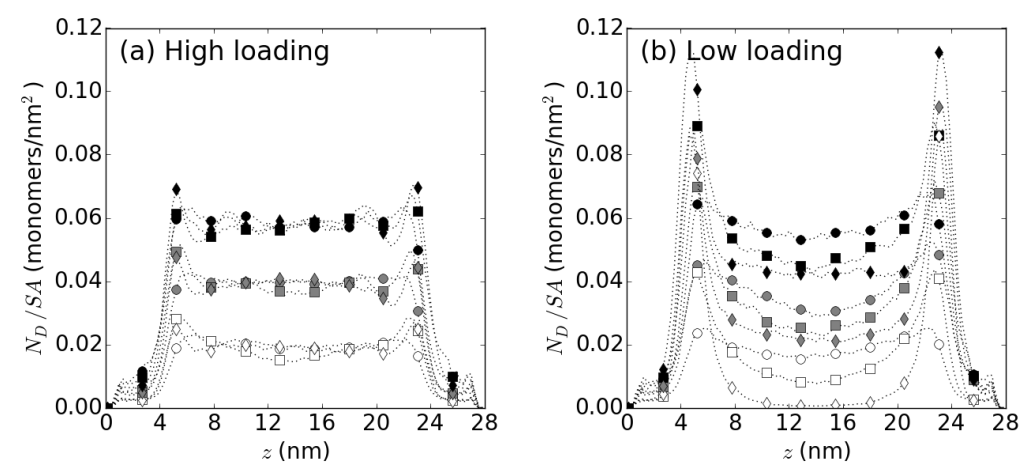

$\bigcirc \bigcirc f_{P L}=0.05, \sigma=1 \sigma_{e} \quad \square \square f_{P L}=0.1, \sigma=1 \sigma_{e} \quad \diamond \diamond f_{P L}=0.2, \sigma=1 \sigma_{e}$

- $f_{P L}=0.05, \sigma=2 \sigma_{e} \quad \square \quad f_{P L}=0.1, \sigma=2 \sigma_{e} \diamond f_{P L}=0.2, \sigma=2 \sigma_{e}$

- $f_{P L}=0.05, \sigma=3 \sigma_{e}$ ॥ $f_{P L}=0.1, \sigma=3 \sigma_{e} \bullet f_{P L}=0.2, \sigma=3 \sigma_{e}$

Figure 3.8: MD simulations of lysozyme in polymer-grafted adsorbents predict that the charged polymers generally exhibit a uniform distribution within the pore at high protein loadings, and can partition towards the surface at low protein loadings. The number density of dextran monomers normalized by the pore surface area, $N_{D} / S$, relative to $z$ position within the pore, is shown for each adsorbent for both high (a) and low (b) protein loadings, with strong electrostatics $\left(\epsilon_{r}=10\right)$. At high protein loadings leading to saturation, all adsorbents have similarly uniform polymer densities. At low protein loadings, the polymers in adsorbents with intermediate and high $f_{P L}$ values partition away from the pore interior and towards the pore surfaces due to favorable interactions with surfacebound protein.

In Figure 3.8a, the loadings are such that each adsorbent is near saturation conditions, with an equilibrium solution concentration of $C \approx 2 \mathrm{mg} / \mathrm{ml}$. The distribution of polymers in the interior of the pore space is relatively uniform for 
all systems. The low number densities at low and high $z$ positions reflect the presence of surface ligands and surface-bound protein that prevent the polymers from accessing these regions of the pore. The different magnitudes of the three groups of curves reflect the three graft densities tested. For each graft density, increasing the polymer charge content beyond $f_{P L}=0.05$ leads to slight peaks in the number density at intermediate $z$ positions of approximately 5 and 23 $\mathrm{nm}$, which exceed the number density in the pore interior by $20 \%$ to $40 \%$. These deviations from a uniform distribution result from favorable interactions between the moderately- and highly-charged polymers with surface-bound protein.

In Figure 3.8b, the protein loadings in each system are sufficiently low that all protein is bound to the surface, which has a concentration between $q_{s}=170$ and $220 \mathrm{mg} / \mathrm{ml}$. Under these conditions the polymer number density deviates more significantly from a uniform distribution when the charge content per polymer exceeds $f_{P L}=0.05$. In these systems, the polymers have a strong preference for the pore surface relative to the interior of the pore due to interactions with the oppositely charged protein on the surface and the absence of protein in the pore. In the adsorbent with $f_{P L}=0.05$ and $\sigma=\sigma_{e}$, the interior pore space is completely devoid of polymers due to this effect. When electrostatics interactions are weaker $\left(\epsilon_{r}=15\right)$, polymer partitioning to the pore surfaces at low loadings is less pronounced but still evident for highly charged adsorbents.

Figure 3.9 shows how changes in the polymer structure with respect to protein loading are governed by the local concentrations of protein on the surface and in the pore space. For simulations of the system with $f_{P L}=0.2, \sigma=\sigma_{e}$, and $\epsilon_{r}=10$ with different loadings (represented by different colors), $N_{D} / S$ versus $z$ is plotted as a function of the local concentrations $q_{s}$ and $c_{p}$ in Figures 3.9a and $3.9 \mathrm{~b}$, respectively. At a low loading that leads to $q_{s}<100 \mathrm{mg} / \mathrm{ml}$ and 
$c_{p}=0$, the polymers exhibit a uniform distribution throughout the interior of the pore. Moderately higher loadings lead to increases in $q_{s}$ while the pore space concentration remains at $c_{p}=0$, due to the greater affinity of protein for the surface versus the polymers. This in turn causes the polymers to partition away from the pore interior and towards the surfaces where they can interact with the adsorbed protein. Finally, at higher protein loadings leading to $c_{p}>$ 0 , the polymers return to the interior of the pore, eventually reaching a nearuniform distribution under saturation conditions. At these high loadings, there is a sufficient number of protein molecules in the pore space to attract the polymers to this region.

When electrostatics are weaker ( $\epsilon_{r}=15$, not shown), the polymers also exhibit some preference for the surface at intermediate loadings, however, the interior pore space is never devoid of polymer. This reflects the weaker affinity of the polymers for surface-bound protein when electrostatics are highly screened. 

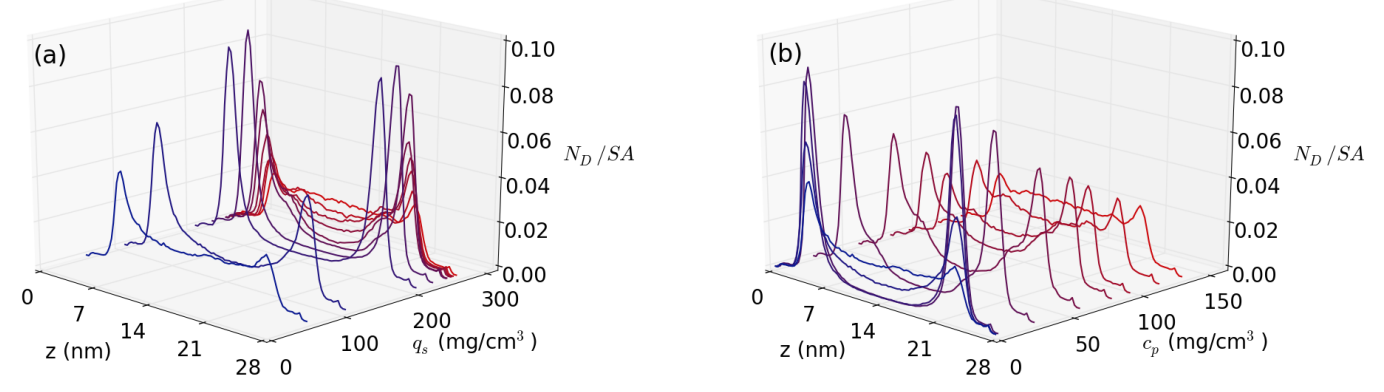

Figure 3.9: MD simulations of lysozyme in polymer-grafted adsorbents predict that the local protein concentrations within the pore can significantly affect the structure of the charged polymers when electrostatic interactions are strong. For the system with $f_{P L}=0.2$ and $\sigma=\sigma_{e}$ and $\epsilon_{r}=10$, the polymer number density normalized by pore surface area, $N_{D} / S$, with respect to $z$ position is plotted as a function of $q_{s}$ (a) and $c_{p}(\mathrm{~b})$. At a low protein loading the polymers exhibit a uniform distribution in the pore interior. At intermediate loadings the polymers partition away from the interior and towards the pore surface due to favorable interactions with surface-bound protein and the absence of protein in the pore interior. At higher loadings the polymers exhibit a more uniform distribution due to favorable interactions with protein in the pore space when the surface is nearly saturated.

The observation that protein diffusivities in the pore space are relatively constant with respect to protein loading justifies the use of a two-phase mass transfer model despite the observed inhomogeneities in the polymer structure under certain conditions. For the system with $f_{P L}=0.2, \sigma=\sigma_{e}$, and $\epsilon_{r}=10$ discussed above, diffusivities were measured at loadings of $N=250$, in which the polymers completely collapse onto the protein-covered surface, $N=300$, in which the polymers are attracted to surface-bound protein but extend somewhat into 
the pore space, and $N=320$, in which the polymers exhibit a uniform distribution throughout the pore space. $D_{p}$ values of $0.37 \pm 0.05,0.31 \pm 0.03$, and $0.31 \pm 0.02 \times 10^{-6} \mathrm{~cm}^{2} / \mathrm{s}$ were measured in these respective systems. The similarity of these results suggests that the two-phase transport model employed in this work, in which protein molecules are either on the surface or in the pore space, is appropriate. According to our model, accounting for slight deviations in $D_{p}$ with respect to polymer structure would have only a very small effect on the predicted adsorption kinetics. However, it is possible that variations in the polymer structure at low loadings could impact the stability of the adsorbed protein, as a recent experiment has shown that for a monoclonal antibody adsorbed on a polymer-grafted cation-exchange resin, an increasing fraction of the bound protein becomes unfolded over time at low protein loadings. [62]

\subsection{Conclusions}

Multi-scale modeling is employed to study how protein adsorption onto polymergrafted IEC adsorbents varies with the charge content per polymer, the polymer graft density, and the strength of electrostatics. Differences in the predicted adsorption capacities for the various systems are driven by different degrees of protein partitioning into the polymer-filled pore space. In general, when electrostatics are strong, pore space partitioning and the effective transport rate both vary approximately linearly with the overall charge content of the polymers, while diffusion in the pore space decreases with respect to the overall charge content. More highly charged systems also lead to more diffuse intraparticle concentration profiles. In systems with a high per-polymer charge content and a relatively low protein loading, the polymer grafts preferentially partition towards the surface 
due to favorable interactions with surface-bound protein. The predicted adsorption equilibria results compare favorably with previous experiments showing that increasing the charge content of a polymer-grafted particle can lead to higher capacities and that the polymers can partially exclude protein from the pore under weak binding conditions.

These results suggest that highly charged polymer-grafted systems can substantially enhance a protein's adsorption capacity and kinetics. For lysozyme, increasing the charge content per polymer and the polymer graft density both lead to comparable enhancements in adsorption properties, according to our model. However, the adsorption of other proteins might be more enhanced by manipulating only one material property, for example, if larger proteins were excluded from the adsorbent pore when the graft density was very high or the individual grafts were only weakly charged. Additional studies on how protein characteristics affect adsorption in polymer-grafted systems would help in designing adsorbents to achieve efficient separations of novel biological products. 


\section{Chapter 4}

\section{Effects of Protein Properties}

\subsection{Introduction}

This chapter describes a multiscale modeling study on the effects of protein properties on adsorption and transport in polymer-grafted IEC resins. The modeling approach introduced previously is extended to lysozyme with different net charges, BSA, and IgG1 in order to predict the macroscopic adsorption behaviors of these proteins and the molecular details of how they interact with charged polymer grafts.

\subsection{Background}

Previous experiments have shown the molecular properties of a protein significantly affect the degree to which polymer-grafted IEC resins can enhance its adsorption equilibria and mass transfer, and the dependence of these enhancements on the solution ionic strength. For example, Stone and Carta used batch uptake experiments to estimate the effective diffusivity of lysozyme, BSA, and 
an IgG1 mAb during adsorption onto experimental cation-exchange resins with either an open pore structure or charged dextran grafts in the pores. While the macroporous resin provided a $D_{e, a p p} / D_{0}$ of approximately 1 or less for each protein, the dextran-grafted resin led to a $D_{e, a p p} / D_{0}>1$ for lysozyme at low salt, $D_{e, a p p} / D_{0}<1$ for BSA over a range of salt concentrations, and $D_{e, a p p} / D_{0}>1$ for IgG1, which increased with the salt concentration. The enhanced transport rates observed for lysozyme and IgG1 indicated that mechanisms besides ordinary pore diffusion contributed to mass transfer in these cases.

Other studies have shown that charged polymer grafts can have either positive or negative effects on the binding capacity, depending on the protein and salt concentration. In another study, Perez-Almodovar et al. found that the commercial polymer-grafted resin Nuvia S provided similarly high capacities for lysozyme and a $\mathrm{mAb}$, while UNOsphere $\mathrm{S}$, a macroporous resin with a similar base matrix, provided a significantly lower capacity for the $\mathrm{mAb}$ than for lysozyme. This suggested the importance of the charged polymers for allowing multi-layer protein adsorption within the pores, which led to high binding capacities for both large and small proteins. [60] On the other hand, Bowes and coworkers found that charged dextran grafts can partially exclude large proteins from the particle pores under high salt concentrations, leading to much larger reductions in capacity than were observed for smaller proteins. [10] The same group also found that increasing the dextran content of a resin led to steric exclusion of lactoferrin from the pores at high salt concentrations unless the ligand density was sufficiently high, whereas the same resin modifications enhanced the binding capacity for lysozyme. [23] The molecular basis for these different adsorption behaviors in polymer-grafted resins is not fully understood, but the variations likely are related to the surface charge distribution and size of the proteins. 
Therefore, this chapter describes the use of multiscale modeling to gain insights into how protein properties affect adsorption and diffusion on both the molecular and macroscopic scales. We extend the multiscale modeling approach described in Chapter 2 to simulate lysozyme with various net charges, BSA, and IgG1. These test systems are chosen both in order to compare our modeling predictions with previous experimental results for similar systems, [20] and to test previously-proposed hypotheses on the relative effects of protein charge and size on the mass transfer rate. [61] Previous experiments have suggested that more highly charged proteins experience enhanced transport in polymer-grafted systems due to the "chain delivery" effect, by which protein diffusion is facilitated by the random thermal motion of the polymer grafts to which they are adsorbed. Experiments have also suggested that the polymer grafts have significant steric exclusion effects on larger proteins, which reduce the effective mass transfer rate. Thus, the polymer grafts have competing effects on transport. These effects are considered at the end of this chapter, which discusses analysis on how the protein's size and charge affect its molecular interactions with the charged polymer grafts as predicted by the MD simulations. Additionally, we aim to test the hypothesis, based on our previous simulations of various systems, that the transport rate generally increases with the amount of protein partitioning into the pore space, which explains the enhanced adsorption kinetics observed in polymer-grafted systems that promote this partitioning. 


\subsection{Methods}

\subsubsection{MD simulation details}

Details of the MD simulations used to determine protein adsorption equilibria and diffusivities are largely the same as those described in Chapter 2. Table 4.1 lists the proteins studied in this work, their relevant physical properties, and the PDB code for the experimental crystal structure used to make the CG protein model. Lysozyme molecules with three different net charges are simulated in order to assess the effects of charge on adsorption of a relatively small, globular protein. These different molecules are referred to as "charge variants" throughout this work, although they are not true protein isoforms having different primary sequences, but only represent different solution $\mathrm{pH}$ values. While net charges of +12 and +4 for lysozyme correspond to extremely acidic and basic buffer conditions, respectively, such conditions may be encountered in some downstream purification steps. Bovine serum albumin (BSA) is simulated in order to study the effects of size, as BSA has approximately 4.5 times the mass of lysozyme and a comparable net charge at $\mathrm{pH}=4.75$ as lysozyme at $\mathrm{pH}=6.5$. Additionally, a monoclonal antibody of the IgG1 subclass is simulated in order to study the industrially-relevant case of a biologic with large molecular mass, non-uniform geometry, and high net charge. 


\begin{tabular}{|c|c|c|c|c|}
\hline Protein & Net charge & Corresponding $\mathrm{pH}$ & Mass $(\mathrm{kDa})$ & PDB Code \\
\hline Lysozyme & +4 & 10 & 14.5 & 1AKI [63] \\
Lysozyme & +8 & 6.5 & 14.5 & 1AKI \\
Lysozyme & +12 & 3 & 14.5 & 1AKI \\
BSA & +9 & 4.75 & 66.5 & 3V03 [64] \\
IgG1 & +54 & 5 & 142.5 & 1HZH [65] \\
\hline
\end{tabular}

Table 4.1: Molecular properties and PDB codes of proteins studied in this work.

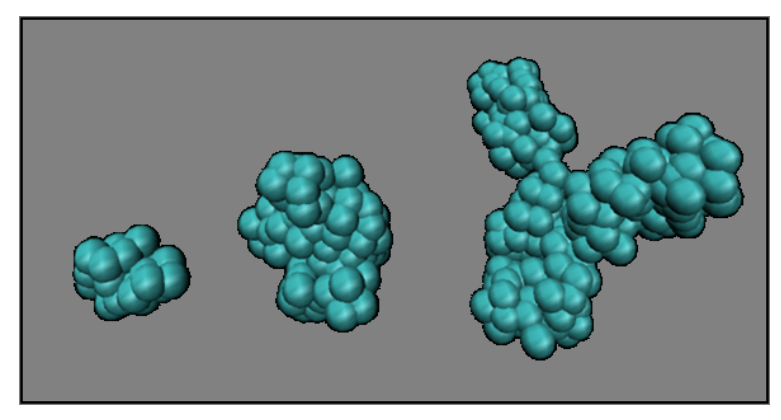

Figure 4.1: Side by side comparison of coarse-grained models of the different proteins studied in this work, lysozyme (left), BSA (center), and IgG1 (right).

CG models were prepared for each protein by the same coarse-graining procedure described in Chapter 2, with $n_{C G}=5$ residues per bead and an elastic network of harmonic bonds between nearby beads used to maintain the structure. Fig. 4.1 shows the CG protein models side by side to illustrate the different sizes of the molecules. Two pre-processing steps were necessary to generate a CG model representative of the protein at the desired solution $\mathrm{pH}$ from the raw PDB file. First, the online homology modeling tool MODELLER (https://salilab.org/modeller/) was used to predict the coordinates of non-hydrogen atoms missing from the PDB file. [66] Second, the online tool $\mathrm{H}++$ (http://biophysics.cs.vt.edu/H++) was 
used to predict the protonation state of titratable amino acid residues at the desired $\mathrm{pH}$ given in Table 4.1. [67] The resulting charge distribution was then used to generate a CG model with $n_{C G}=5$ by the procedures described in Chapter 2. Each type of protein was simulated in an open macropore and in a dextran grafted pore with a per-polymer charge content of $f_{P L}=0.05$ and graft density of $\sigma=\sigma_{e}$. Snapshots from MD simulations of each protein in the polymer-grafted system under saturation conditions are shown in Fig. 4.2. Each of these polymergrafted systems includes an external void region adjacent to the adsorbent pore, which as discussed in Chapter 2, allows for convenient calculation of the concentration of the free solution, $C$, in equilibrium with the adsorbed phase. Similarly, a void region was included in simulations of BSA and IgG1 in the macroporous system in order to facilitate calculation of $C$ in the free solution in equilibrium with the macropore. The void was not included in simulations of the lysozyme charge variants in the macroporous system, as these relatively small proteins can diffuse through the pore space without interacting with the underlying pore surfaces, and therefore $C$ may be approximated as the pore space concentration, $c_{p}$. Conversely, in the BSA and IgG1 systems the pore space is relatively crowded due to the large size of the proteins, and therefore the void region is included to calculate $C$. 

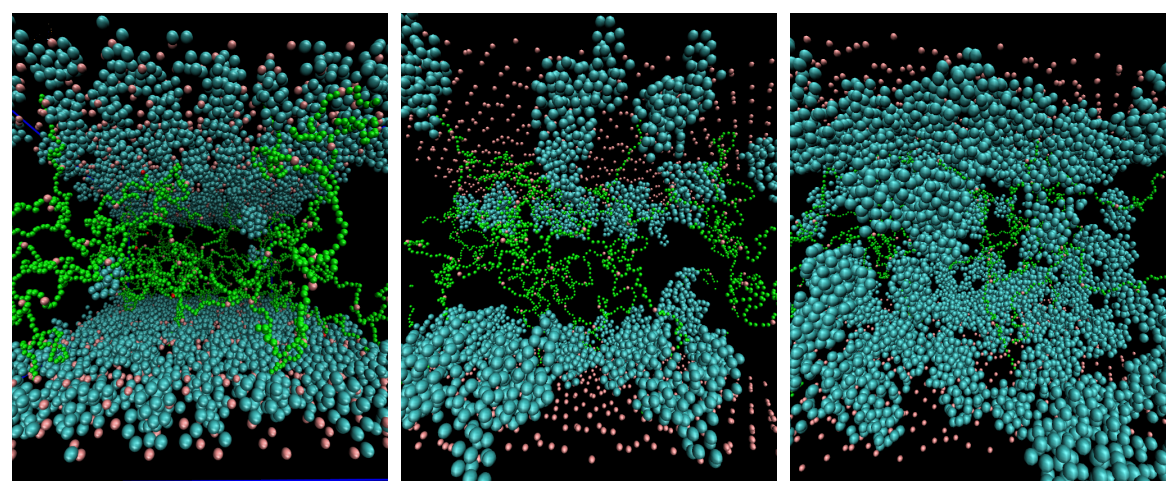

Figure 4.2: Snapshots from MD simulations of lysozyme (left), BSA (middle), and IgG1 (right), in a polymer-grafted pore under saturation conditions, illustrating the different degrees to which the various proteins partition into the pore space.

The lysozyme charge variants were simulated with two different values for the inner dielectric that screens electrostatic interactions, $\epsilon_{r}=10$ and $\epsilon_{r}=15$, which represent strong and weak binding conditions, respectively. BSA was simulated with $\epsilon_{r}=12$ and IgG1 with $\epsilon_{r}=15$, as it was determined through a series of trial simulations that lower $\epsilon_{r}$ values led these proteins to form dimers and larger aggregates in solution rather than partition into the adsorbent pore. Because the excluded volume of each CG protein bead is described by a purely repulsive LJ potential, any observed protein-protein attraction is driven by electrostatics, which is inconsistent with the physical systems. The aggregation was partially reduced by equilibrating the system one protein molecule at a time, indicating that the aggregation process is at least somewhat kinetically-limited. However, some aggregation is still observed using long equilibration times of $180 \mathrm{~ns}$ for each added protein, which was at the upper limit of computational feasibility. While the different $\epsilon_{r}$ values used for the various proteins unfortunately do not allow for a straightforward interpretation of how favorable binding conditions 
are, qualitative comparisons with experimental results can still be made.

Local protein concentrations and diffusivities are calculated by the same approaches described in Chapter 2. However, a different definition of surface adsorption is necessary for BSA and IgG1 due to their larger size relative to the pore space. For each of these proteins, a given molecule is considered surfaceadsorbed when the separation between any CG bead of the molecule and the nearest surface ligand is $1.5 \mathrm{~nm}$ or less, and is considered to be in the pore space when this separation is greater than $1.5 \mathrm{~nm}$. This cutoff is beyond the large first peak in the distribution of protein-surface ligand distances corresponding to surface-bound molecules, as shown in Fig. 4.3. All diffusivities were calculated according to the Einstein relation, with the MSD slope evaluated between $\Delta t=$ 30 and 60 ps. In this region the average MSD of each protein, both on the surface and in the pore space, was linear. 


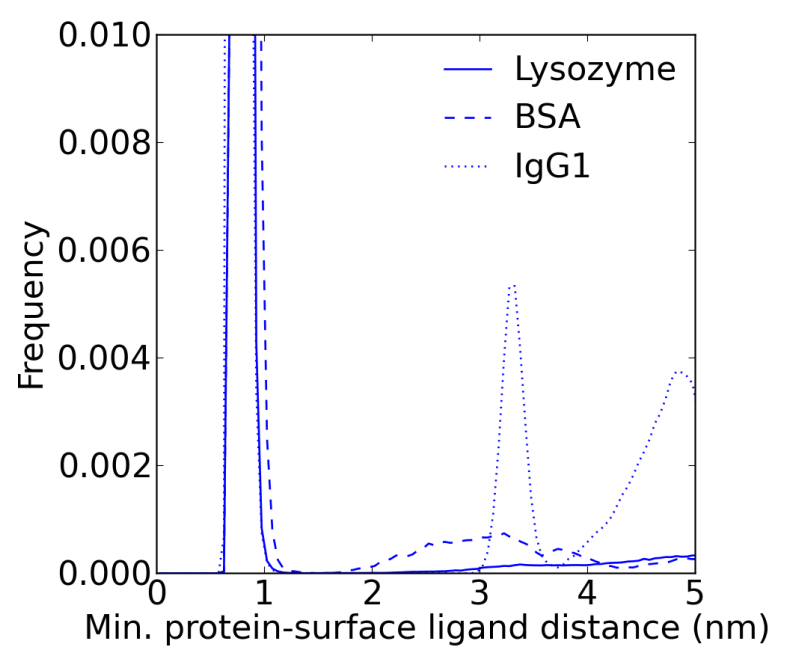

Figure 4.3: For each protein, the histogram of minimum distances to the nearest surface ligand exhibits a large peak corresponding to surface-bound protein. Based on this information, a protein molecule is considered adsorbed on the surface when this distance is $1.5 \mathrm{~nm}$ or less, and in the pore space when the distance is greater than $1.5 \mathrm{~nm}$.

\subsection{Results}

\subsubsection{Adsorption Equilibria}

Adsorption isotherms determined by MD simulation suggest that the capacity of the polymer-grafted pore increases with the charge of the protein and decreases with its size. The effect of charge is illustrated in Fig. 4.4, which shows isotherms calculated for the lysozyme charge variants in both the dextran-grafted pore and the open macropore. As seen in comparing the $\hat{q}$ vs. $C$ isotherms in Figs. 4.4a and $\mathrm{d}$, when electrostatics are strong $\left(\epsilon_{r}=10\right)$, the polymer grafts enhance the overall capacity for only the +12 charge variant (by 20\%) and provide a comparable 
capacity to that of the open macropore for the less charged variants. For both adsorbents and both $\epsilon_{r}$ values, total capacities are generally lowest for the +4 charge variant, as is expected for cation exchange chromatography. The capacities are comparable for the +8 and +12 charge variants, which suggests that the adsorbed concentration may be at a steric limit.

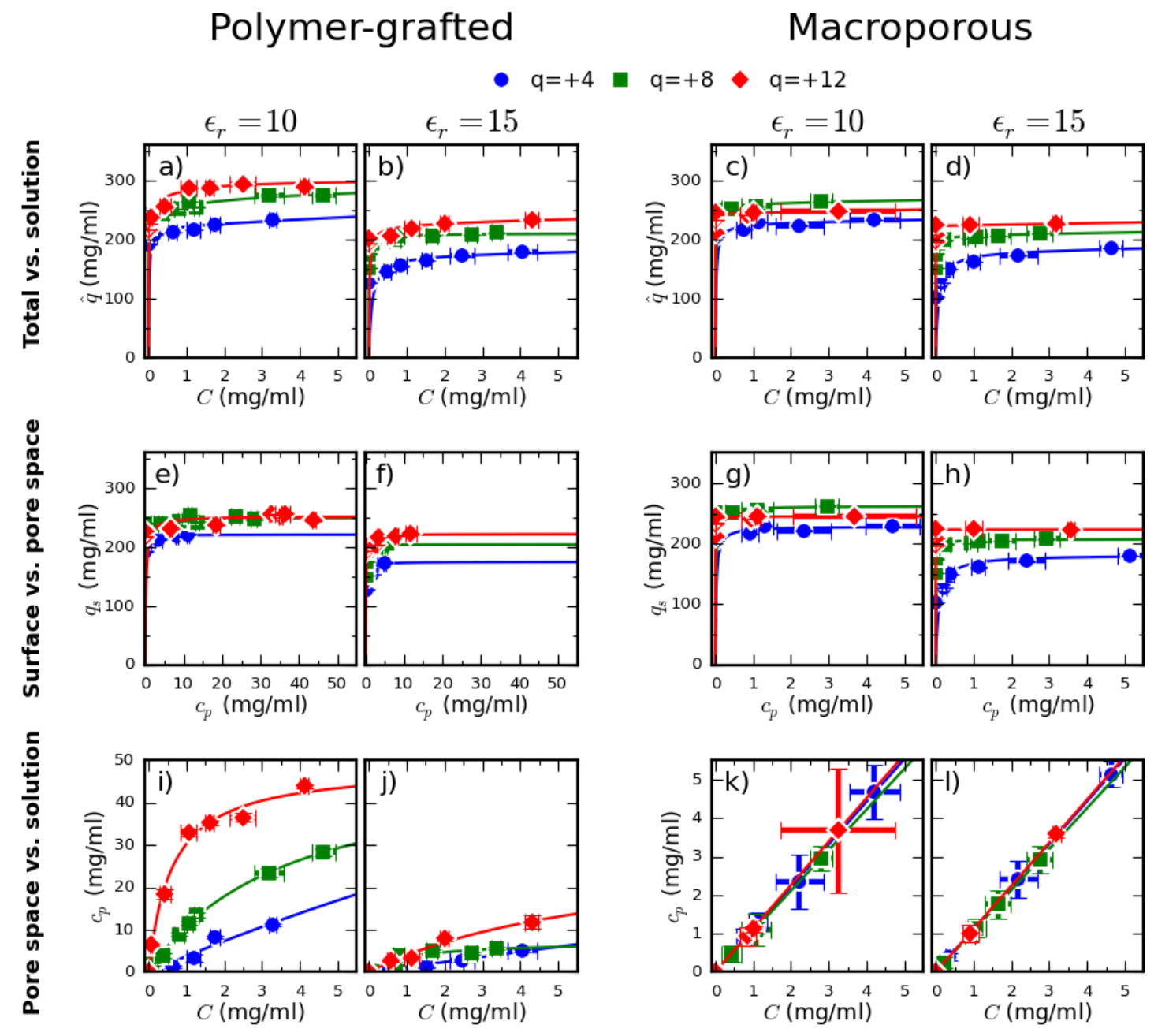

Figure 4.4: Adsorption isotherms of lysozyme charge variants predicted by MD simulation show that for the polymer-grafted system, a higher net charge of the protein leads to greater pore space partitioning and a higher overall capacity, particularly when electrostatics are strong $\left(\epsilon_{r}=10\right)$. 
The predicted local isotherms demonstrate that differences in the total capacities of the polymer-grafted and macroporous systems for lysozyme are due to different amounts of protein partitioning into the pore space. As seen in comparing the $q_{s}$ vs. $c_{p}$ isotherms in Figs. 4.4e-h, the polymer-grafted and macroporous systems generally exhibit comparable surface capacities for a given charge variant and $\epsilon_{r}$ value. However, the $c_{p}$ vs. $C$ isotherms given in Figs. 4.4i-1 show that partitioning into the polymer-grafted pore can deviate significantly from the linear isotherm expected for an open-macropore. In the polymer-grafted pore with $\epsilon_{r}=10$, the $c_{p}$ vs. $C$ isotherms become increasingly rectangular and exhibit higher saturation capacities, $c_{p}^{\max }$ (i.e. $c_{p}$ at $C=2 \mathrm{mg} / \mathrm{ml}$ ), as lysozyme's charge increases from +4 to +12 . This increase in $c_{p}^{\max }$ is most responsible for the increase in the total capacity with respect to lysozyme's charge seen in Fig. 4.4a, though slight increases in the surface capacity (Fig. 4.4e) also contribute.

Partitioning into the polymer-filled pore space is greatly reduced when electrostatics are weaker (with $\epsilon_{r}=15$ ), as seen in Fig. 4.4j. Under these conditions, the protein net charge has relatively little effect on pore space partitioning. As previously mentioned, the surface capacities of the polymer-grafted and macroporous systems are constant for $\epsilon_{r}=15$, indicating that even when protein affinity for the polymer ligands is weak, the protein can still access the interior of the pore and adsorb onto the surface. Therefore, the simulations suggest that lysozyme is not sterically hindered by the neutral polymer segments, consistent with our results in Chapter 2 and the experimental work of Bowes et al. on lysozyme adsorption in resins with different dextran graft densities. [23]

Adsorption isotherms for BSA shown in Fig. 4.5 demonstrate that protein can be excluded from the polymer-grafted pore under certain conditions. For both the polymer-grafted and macroporous systems, the overall capacity (Fig. 4.5a) 
is primarily determined by the capacity of the surface (Fig. 4.5b). The overall capacity of the polymer-grafted pore is approximately $20 \%$ lower than that of the open macropore due to a similar reduction in the amount of surface adsorption. This indicates that the dextran grafts partially exclude BSA from binding to the underlying charged surface. As shown in Figs. 4.4e-h, the grafts do not have a significant effect on lysozyme's surface capacity, suggesting that surface adsorption of the larger BSA molecules is reduced due to steric hindrance by the polymers. For these systems, BSA exhibits the same amount of partitioning into the polymer-grafted pore and the open macropore. The low affinity of BSA for the charged polymer phase may also be attributed to steric hindrance of the neutral dextran segments, as lysozyme, which has a similar net charge but $1 / 4$ th the mass of BSA, exhibits greater affinity for the charged polymers under identical conditions, as discussed in Chapter 2.
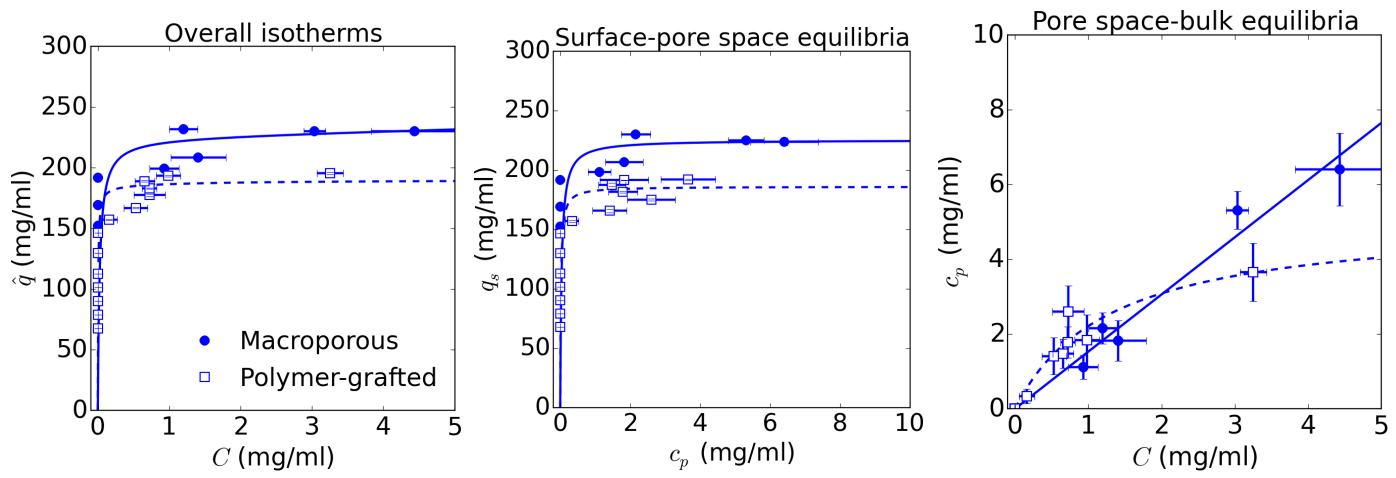

Figure 4.5: Adsorption isotherms of BSA predicted by MD simulations show that the polymer grafts lead to slight decreases in both BSA adsorption to the underlying surface and in its partitioning into the pore space, relative to the macroporous system.

Adsorption isotherms predicted for IgG1, shown in Fig. 4.6, demonstrate that 
the steric effects of the polymer grafts on the adsorption of a large protein can be overcome if the protein is sufficiently charged. The $\hat{q}$ vs. $C$ isotherms given in Fig. 4.6a show that the polymer grafts provide a slight increase (5\%) in the overall adsorption capacity. Although surface adsorption of the mAb in the polymergrafted pore is slightly lower than in the open macropore, shown in Fig. 4.6b, the substantial partitioning into the polymer phase leads to the net improvement in the overall capacity. This suggests that the highly charged mAb has sufficient affinity for both the polymer-filled pore space and the underlying polymer-grafted surface to overcome the exclusion effects of the neutral dextran segments, unlike the smaller, weakly charged BSA molecules.
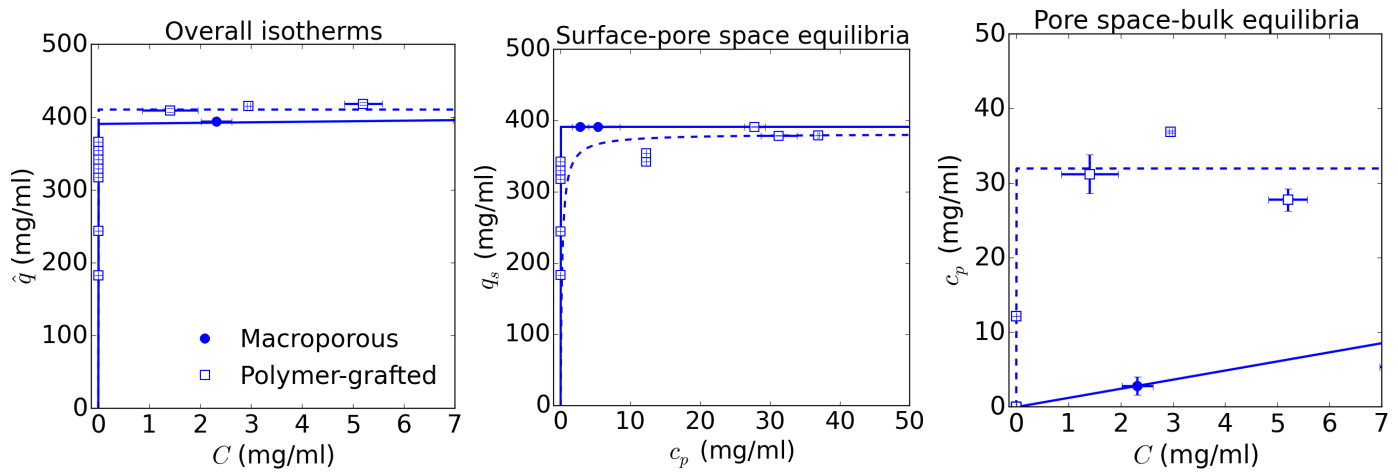

Figure 4.6: Adsorption isotherms of IgG1 predicted by MD simulations show that the charged polymer grafts lead to increased protein partitioning into the pore space compared to the macroporous system, though this has a relatively small effect on the overall adsorption capacity, which is dominated by the capacity of the underlying surface.

To summarize the adsorption equilibria results predicted by MD, partitioning into the polymer-filled pore space generally increases with respect to protein charge while surface adsorption is constant of this variable, leading to a net 
increase in the overall capacity. Additionally, the BSA and IgG1 results show that the polymer grafts can reduce the adsorption of larger proteins, unless the protein is sufficiently charged.

\subsubsection{Diffusivities}

Diffusivities predicted by MD simulation demonstrate that the protein's size and charge can significantly affect its mobility. Fig. 4.7 shows average diffusivities of protein within the pore space, $D_{p}$, and adsorbed onto the surface, $D_{s}$, for the lysozyme charge variants with relatively strong $\left(\epsilon_{r}=10\right)$ and weak $\left(\epsilon_{r}=15\right)$ electrostatic interactions. As shown in Fig. 4.7a, with $\epsilon_{r}=10$, the lysozyme variants with low and moderate net charges of +4 and +8 diffuse approximately $30 \%$ slower than the protein does in free solution (shown in the dashed line), while diffusion of the +12 variant is slowed by approximately $70 \%$. When $\epsilon_{r}=15$, all the charge variants exhibit a similar reduction in $D_{p}$ of $30 \%$ to $40 \%$. 

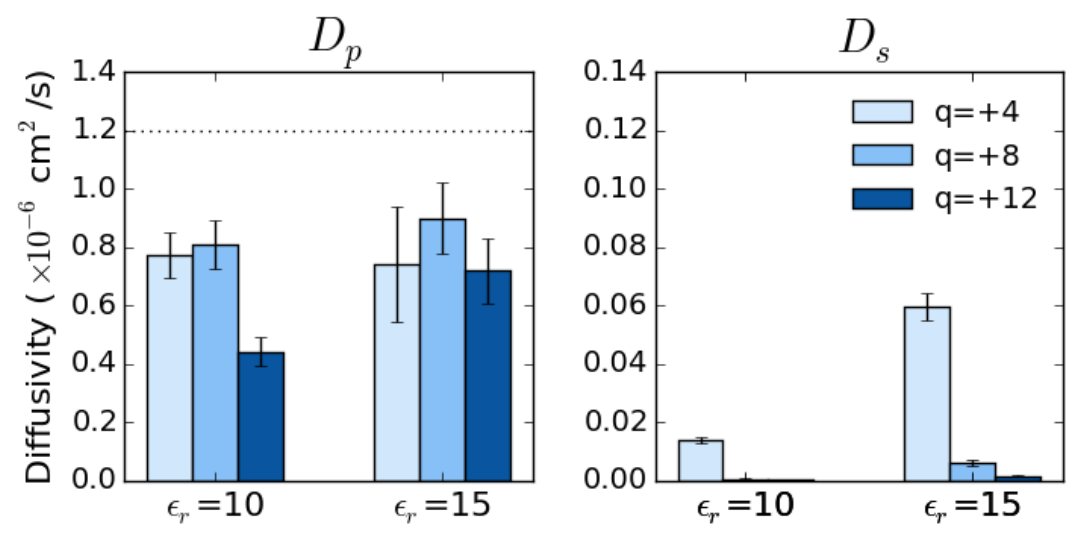

Figure 4.7: Average diffusivities of lysozyme charge variants within the polymerfilled pore, $D_{p}$, and on the surface, $D_{s}$, for different electrostatic strengths, as predicted by MD simulations. $D_{p}$ is significantly lower for the +12 charge variant compared to the other charge variants when electrostatics are strong $\left(\epsilon_{r}=10\right)$, while $D_{s}$ is significantly higher for the +4 variant, for both strong and weak electrostatics. 

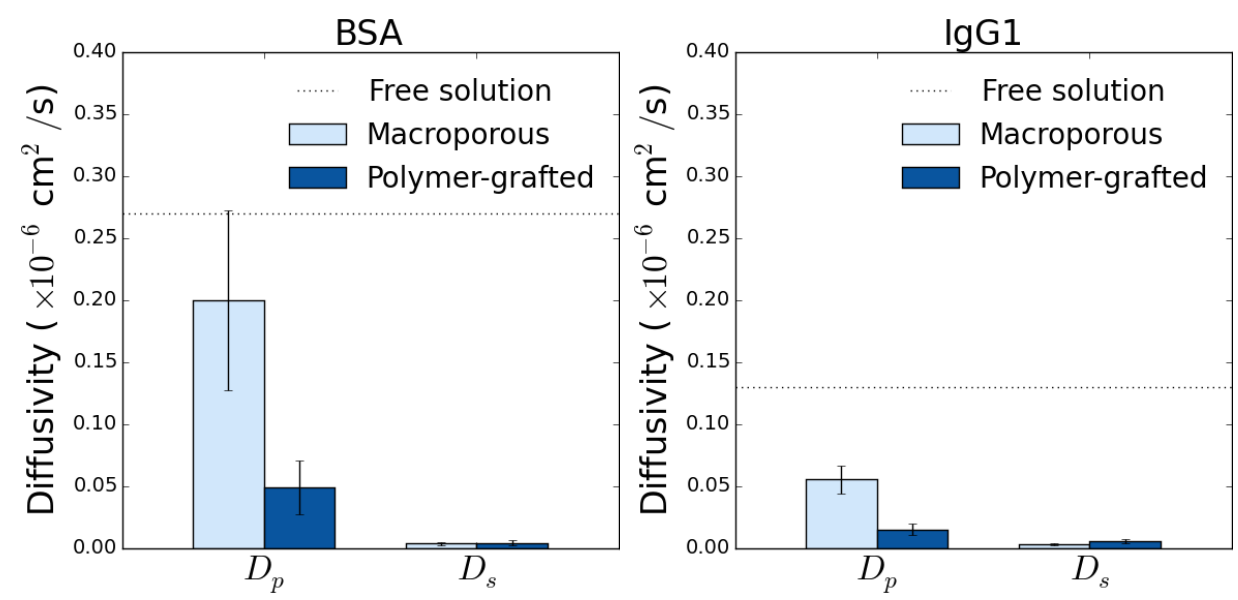

Figure 4.8: Average diffusivities of BSA (left) and IgG1 (right) on the surface and in the pore space of macroporous and polymer-grafted pores, as predicted by MD simulations. Both proteins exhibit similar reductions in diffusion relative to $D_{0}$ (shown in the dashed line) when in the polymer-filled pore space or when adsorbed onto the surface.

Diffusion of lysozyme on the underlying surface is highly dependent on the binding strength to the surface, which is determined by both the charge of the protein and the strength of electrostatics. As shown in the $D_{s}$ values plotted in Fig. 4.7b, for both $\epsilon_{r}=10$ and $\epsilon_{r}=15$, the +4 variant diffuses at least one order of magnitude faster than the more highly charged variants, due to weaker surface attraction because of its low net charge. Similarly, $D_{s}$ for a given charge variant is roughly an order of magnitude higher for $\epsilon_{r}=15$ vs. $\epsilon_{r}=10$, due to weaker binding to the surface when electrostatics are more significantly screened. In general, lysozyme diffuses 1 to 3 orders of magnitude slower on the surface than within the polymer-filled pore space. The lower mobility on the surface reflects the stronger adsorption that occurs there, due to the higher density of ligands on the surface versus attached to the polymer grafts. 
Fig. 4.8 shows the predicted diffusivities of BSA and IgG1, which like those of lysozyme, depend strongly on the adsorption state of the protein. For both proteins, $D_{p}$ in the macroporous system is less than the calculated free solution diffusivity, $D_{0}$, as molecules in the pore space interact with surface-bound molecules due to their large size. This differs from the behavior of the smaller lysozyme, which can freely diffuse through the pore space even under saturation conditions. Though beyond the scope of this study, this effect would be expected to decrease as the size of the macropore increases, allowing more space for pore diffusion of large molecules.

In the polymer-grafted systems, $D_{p}$ of BSA and IgG1 is further reduced by interactions with the charged polymer grafts, by approximately $75 \%$ relative to $D_{p}$ in the macroporous system for each protein. Additionally, each protein diffuses on the surface approximately 2 orders of magnitude more slowly than in free solution. This relatively small reduction affects the predicted macroscopic adsorption kinetics as discussed in the next section. We note that the calculated free solution diffusivities of $0.27 \times 10^{-6} \mathrm{~cm}^{2} / \mathrm{s}$ for BSA and $0.13 \times 10^{-6} \mathrm{~cm}^{2} / \mathrm{s}$ for IgG1 are lower than reported experimental measurements $\left(0.61 \times 10^{-6} \mathrm{~cm}^{2} / \mathrm{s}\right.$ for BSA and $0.37 \times 10^{-6} \mathrm{~cm}^{2} / \mathrm{s}$ for IgG1). [68] This suggests that the Langevin dynamics coupling strength of $\tau_{t}=0.7 \mathrm{ps}$, which provided a good approximation for solvent's effects on lysozyme's diffusion, is overly strong for modeling how these larger proteins would diffuse in free solution. The Langevin dynamics integration scheme essentially imposes a random frictional force (inversely proportional to $\tau_{t}$ ) on all particles in the system. This leads to a dampening of translational diffusion (and other kinetic properties) that increases with the number of atoms in the solute, as we have shown previously. [33] This artifact is evident in the observation that increasing the solute size leads to a reduction in $D_{0}$ predicted by 
our model that exceeds the reduction predicted by the Stokes-Einstein equation. Despite this limitation, the $D_{p}$ values discussed above are useful for qualitative study of how the charged polymers affect a protein's diffusion relative to its free solution diffusivity predicted using the same MD simulation approach.

\subsubsection{Adsorption kinetics}

Transient adsorption of the various proteins over longer scales was studied using mass transfer simulations based on our MD results, as described in Chapter 2. Intraparticle concentration profiles predicted by these simulations show that the shape of the adsorption front into the particle can vary significantly with the properties of the protein. Fig. 4.9 shows the intraparticle concentration profiles predicted for the lysozyme charge variants (with $\epsilon_{r}=10$ ), BSA, and IgG1 in the macroporous and polymer-grafted systems at time $t=50 \mathrm{~s}$. At this time each system is only partially saturated, which allows one to see the qualitative shape of the adsorption front. For lysozyme in both types of pores, the adsorption front of the +4 charge variant is fairly diffuse, as shown in Fig. 4.9 a, while the +8 and +12 charge variants exhibit sharp fronts, seen in Figs. $4.9 \mathrm{~b}$ and c. The further advancement of the front in the polymer-grafted systems indicates accelerated transport due to the charged polymers. As shown in Figs. $4.9 \mathrm{~d}$ and e, both BSA and IgG1 exhibit diffuse adsorption fronts that advance at comparable rates in the two different adsorbents. 

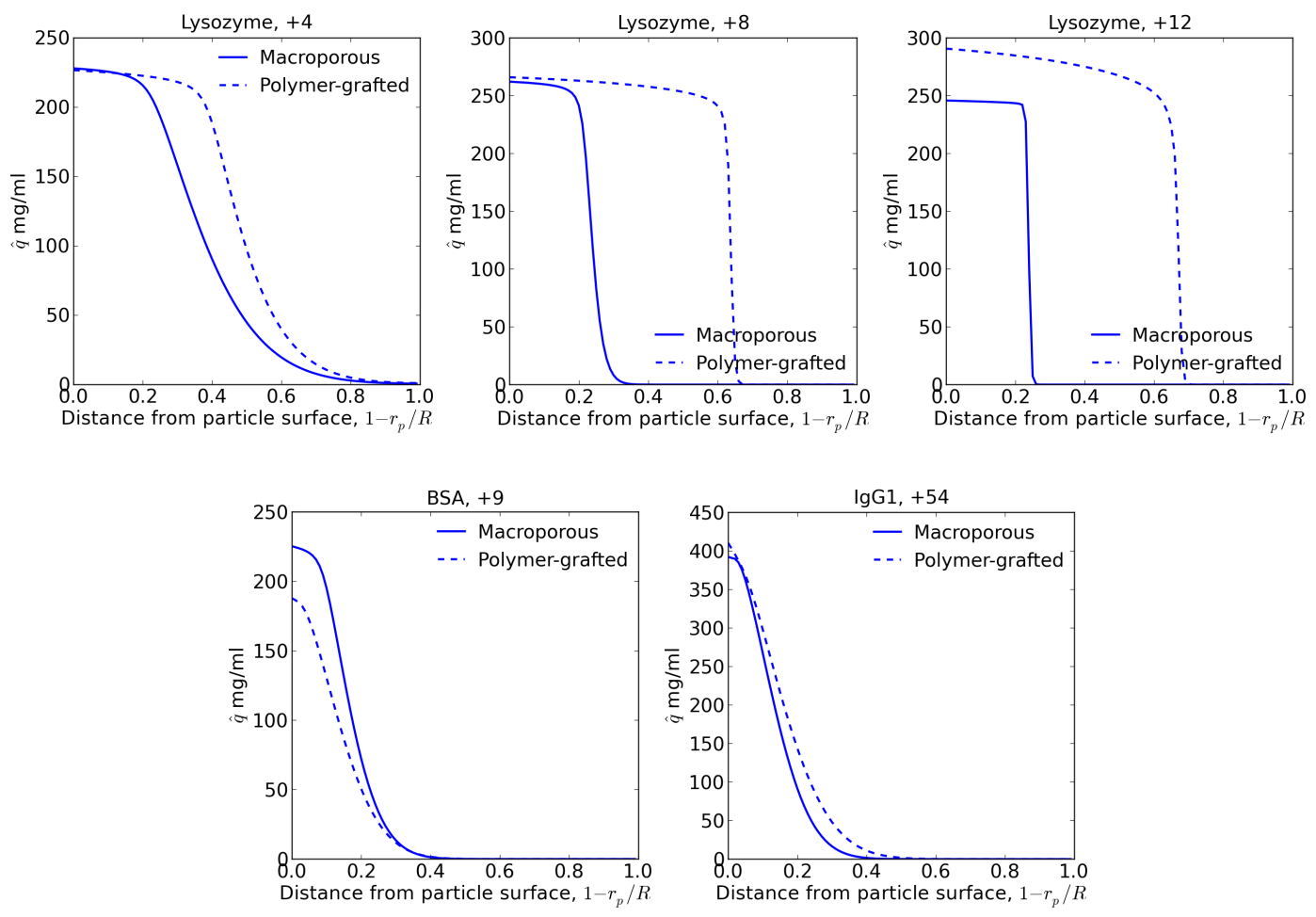

Figure 4.9: Intraparticle concentration profiles for lysozyme charge variants (top row), BSA (bottom left), and IgG1 (bottom right), as predicted by multiscale simulation of mass transfer, show that the shape of the adsorption front can vary from sharp to diffuse, depending on the properties of the protein.

The qualitative differences in the shapes of the adsorption fronts are closely related to the protein's surface diffusion. Comparing simulated $D_{s}$ and $D_{0}$ values reveals that for the proteins with diffuse fronts (lysozyme with a charge of +4 , BSA, and IgG1), $D_{s}$ is approximately 2 orders of magnitude lower than $D_{0}$, while for the proteins with sharper fronts (lysozyme with a charge of +8 and +12 ), $D_{s}$ is 3 to 4 orders of magnitude lower than $D_{0}$. Additionally, trial simulations of lysozyme with +4 , BSA, and IgG1 in which $D_{s}$ was reduced by 2 orders of magnitude (not shown) lead to sharper adsorption fronts. 
The observed relationship between surface diffusion and the intraparticle concentration profile suggests that the mass transfer mechanism transitions from pore diffusion to parallel diffusion as $D_{s}$ increases. The diffuse profiles seen when $D_{s}$ is relatively high are consistent with the parallel diffusion model, in which the protein diffuses both on the surface and in the pore space. [69] In this case the total mass transfer flux is given by $J_{T}=-D_{s} \nabla q-D_{p} \nabla c_{p}$, with the first term contributing significantly to the overall flux due to the combination of high $D_{s}$ and a large $\nabla q$ driving force resulting from the high capacity of the surface. On the other hand, the sharp profiles observed with low $D_{s}$ are consistent with the pore diffusion model, in which transport occurs primarily in the pore space fluid and the flux expression reduces to $J_{T}=-D_{p} \nabla c_{p}$ (or as conventionally written in less-detailed models, $\left.J_{T}=-D_{e} \nabla C\right)$.

Figs. 4.10a and b show that lysozyme's transport rate, as quantified by the ratio $D_{e, a p p} / D_{0}$, can vary significantly with respect to the pore structure, the protein's charge, and the strength of electrostatics. $D_{e, a p p}$ is estimated by fitting the analytical solution of the pore diffusion model to the batch uptake curve predicted by mass transfer simulation, as described in Chapter 2. When electrostatics are strong $\left(\epsilon_{r}=10\right)$, the polymer-grafted system provides a faster transport rate than the open macropore for all charge variants due to the larger $\nabla c_{p}$ driving force resulting from the greater partitioning of the protein into the polymer-filled pore space. Accordingly, these enhancements in $D_{e, a p p} / D_{0}$ are greatest for the +8 and +12 charge variants, which exhibit the most pore space partitioning as seen in Fig. 4.4. In contrast, when electrostatics are weaker $\left(\epsilon_{r}=15\right)$ the transport rate in the polymer-grafted system is comparable to or lower than the rate in the macropore for each charge variant. This reflects both the similar $\nabla c_{p}$ driving forces of the two adsorbents, as the polymers do not greatly improve partitioning into 
the pore space under weak binding conditions, as well as the slightly higher $D_{s}$ values observed in the macroporous system (not shown).
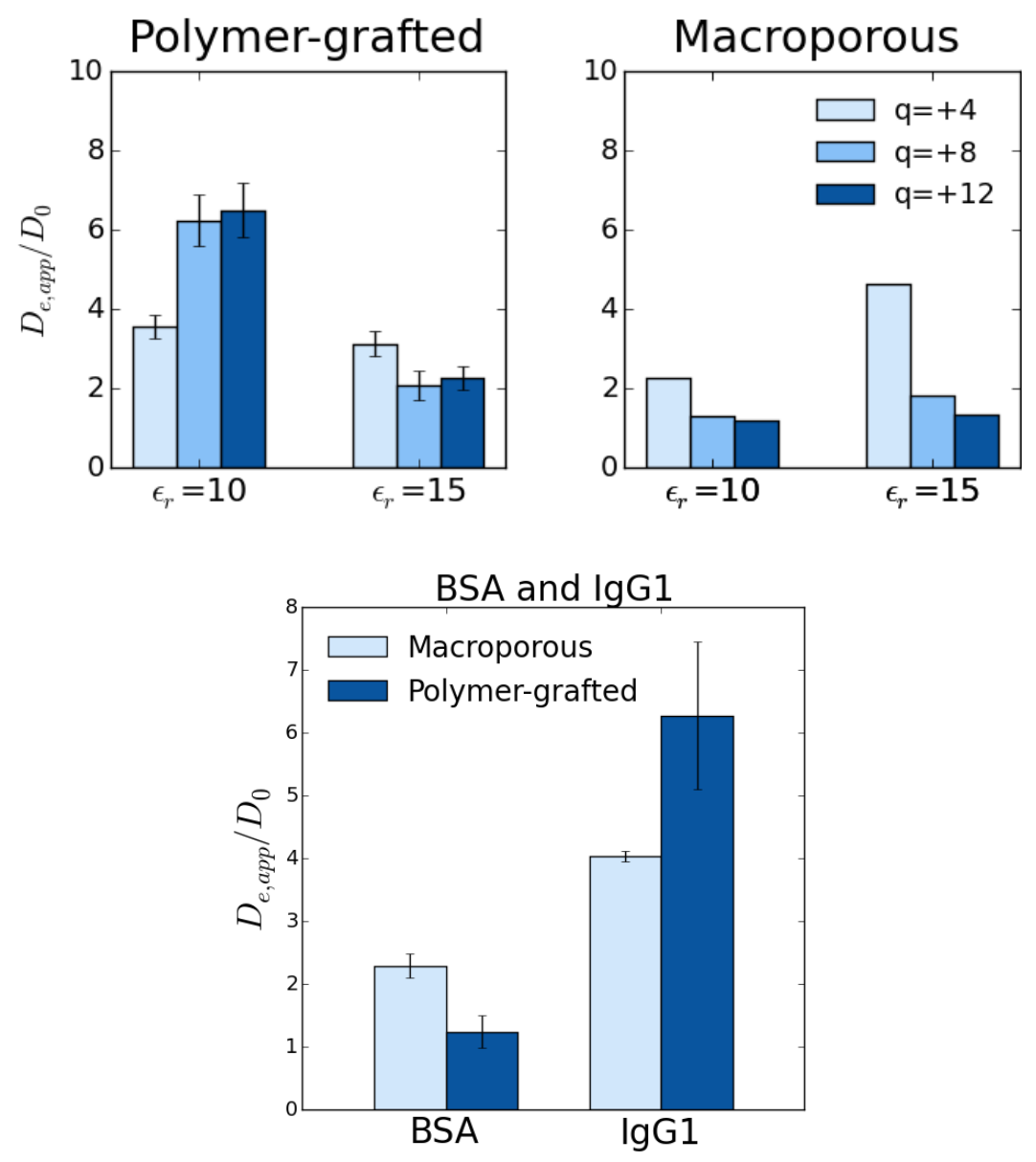

Figure 4.10: Effective transport rate, as determined by $D_{e, a p p} / D_{0}$, of lysozyme charge variants with different electrostatic strengths in the polymer-grafted system (top left) and in the macroporous system (top right), and of BSA and IgG1 in both systems (bottom), as predicted by multiscale simulation of mass transfer. $D_{e, a p p} / D_{0}$ of lysozyme in the polymer-grafted system increases with the protein's charge when electrostatics are strong. The polymer grafts hinder transport of BSA and enhance transport of IgG1 relative to their respective rates in the macroporous system. 
Predicted transport rates for BSA and IgG1 are shown in Fig. 4.10c, which illustrate that charged polymer grafts can actually hinder diffusion of a large protein unless it has a sufficiently high charge. BSA exhibits a $D_{e, a p p} / D_{0}$ of 1.2 (0.2) in the polymer-grafted system, which is qualitatively consistent with the values of $D_{e, a p p} / D_{0}<1$ observed for this protein in the experimental material over a range of ionic strengths. [20] The adsorption isotherms shown in Figs. 4.5 provide evidence that BSA's slow mass transfer rate results from its low level of partitioning into the polymer-grafted pore space. On the other hand, IgG1 exhibits a $D_{e, a p p} / D_{0}$ of $6.3(1.2)$ in the polymer-grafted system, again in qualitative agreement with the enhancements observed experimentally. [20] The adsorption isotherms shown in Figs. 4.6 suggest that this enhancement is due to significant partitioning of IgG1 into the pore space. Again, this occurs despite the mAb's large size because its high net charge allows for favorable interactions with the polymers.

The predicted $D_{e, a p p} / D_{0}$ values in the macroporous system are $2.3(0.3)$ for BSA and $4.0(0.1)$, which exceed the experimental results of $D_{e, a p p} / D_{0}<1$ observed for both proteins in a similar macroporous resin over a range of ionic strengths. [20] These deviations suggest that surface diffusion of BSA and IgG1 is unphysically high in our simulations. The trial mass transfer simulations mentioned above, in which $D_{s}$ was decreased by 2 orders of magnitude, led to approximately $50 \%$ lower $D_{e, a p p} / D_{0}$ values for both BSA and IgG1, in addition to sharper adsorption fronts. This suggests that the $D_{s}$ values predicted by the current molecular models are at least 2 orders of magnitude higher than the physical surface diffusivities. This discrepancy may be related to our idealized model for the pore consisting of two flat surfaces. While this model does include structural heterogeneity over short scales on the order of lysozyme's size, larger scale structural 
features of the pore surface that are not represented here could affect the surface adsorption and mobility of larger protein molecules. Despite this limitation, the model predictions that the polymer grafts enhance the transport of IgG1 but not BSA do support the experimental trends.

\subsubsection{Protein-polymer interactions}

The MD simulations predict that the protein's charge and size significantly affect the number of charged polymer ligands with which it interacts. Figs. 4.11a and $\mathrm{b}$ show histograms of the average number of polymer ligands with which a protein molecule in the pore space is in contact for the lysozyme charge variants. Lysozyme's behavior varies significantly with its net charge and the strength of electrostatics. For the +8 and +12 variants, stronger electrostatics $\left(\epsilon_{r}=10\right)$ favor more multivalent interactions between protein molecules and the charged polymers, with 2 contacts observed most often. Under these same conditions, the +4 variant most often has 0 contacts with the polymer ligands, though it has 1 or more contacts for significant portions of the time as well. When electrostatics are weaker $\left(\epsilon_{r}=15\right)$, the most frequently observed state of each charged variant is unadsorbed, i.e. 0 close contacts with the polymer ligands.

Fig. 4.11c shows histograms of the number of close contacts that BSA, IgG1, and lysozyme (with +8 charge and $\epsilon_{r}=10$ ) have with polymer ligands. As shown, lysozyme and IgG1 exhibit similar preferences for multivalent interactions, with 2 contacts the most common state. However, BSA is most often in an unadsorbed state. These results suggest that the protein's surface charge density has a larger effect than its size on the number of interactions it makes with the polymers. While BSA might be expected to be in close contact with more polymer ligands than lysozyme by virtue of its larger size, the opposite is observed. Not only is 
BSA disfavored from entering the pore space, as seen in the adsorption isotherms in Fig. 4.5, but also the protein that does partition into the pore space does not interact favorably with the charged polymers.
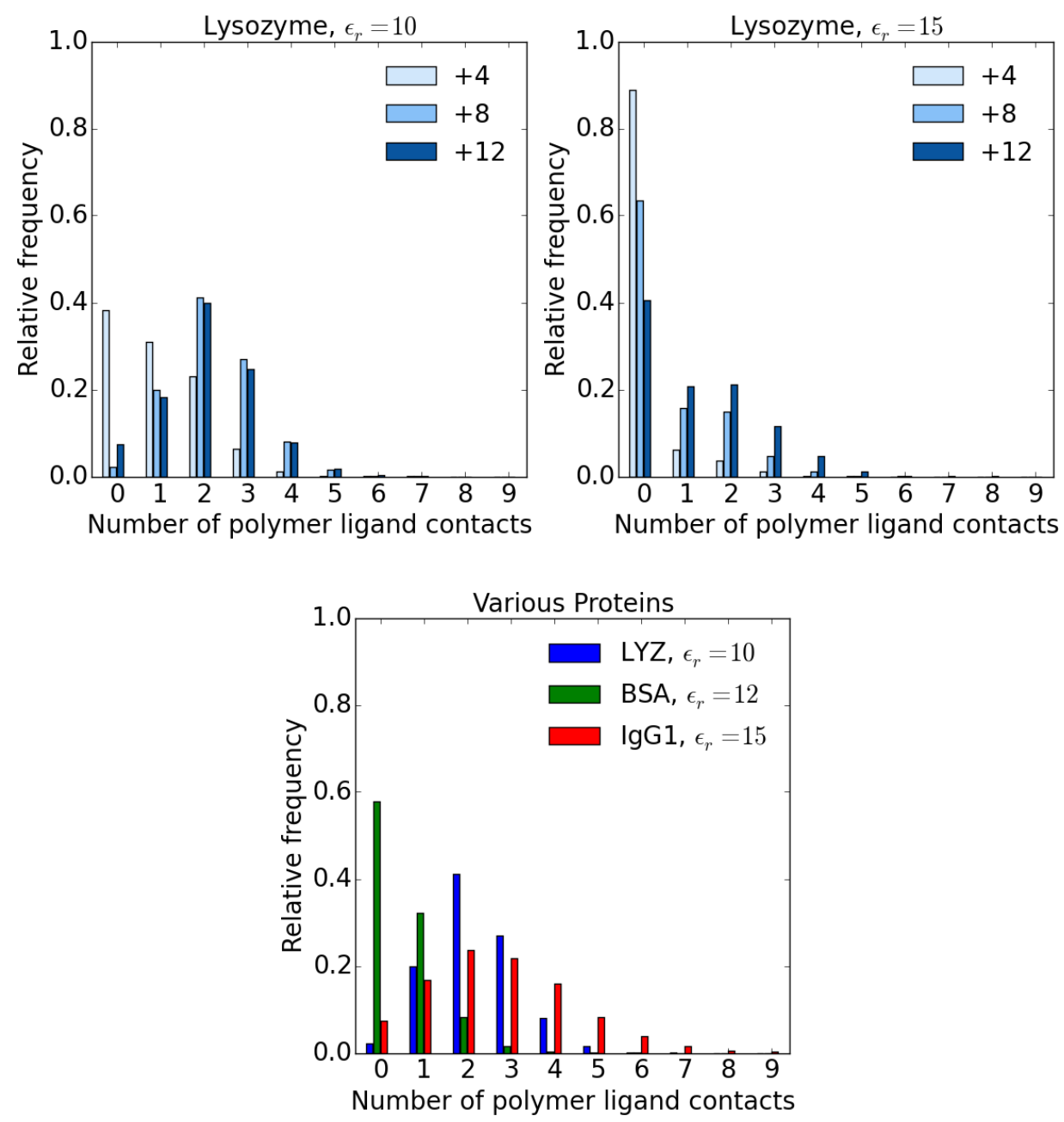

Figure 4.11: Histograms of the average number of polymer ligands with which the protein in the pore space is in contact, for lysozyme charge variants with $\epsilon_{r}=10$ (top left) and $\epsilon_{r}=15$ (top right), and various proteins (bottom). The frequency of observing two or more polymer ligand contacts increases with the size and charge of the protein. 
Additional analysis sheds light on the relationship between the protein's mobility in the pore space and its interactions with the charged polymers. To quantify this, we calculated the net displacement of each molecule in the pore space over a given time period as a function of the average number of polymer ligand contacts during that period. The heat maps plotted in Fig. 4.12 show the relative frequency of observing a given displacement and number of contacts for the lysozyme charge variants (with $\epsilon_{r}=10$ ), BSA, and IgG1. The length of the time window used to measure a molecule's displacement was $60 \mathrm{~ns}$ for lysozyme and $30 \mathrm{~ns}$ for the larger proteins, as these times extend in the linear region of the MSD curves and provide sufficient sampling. 

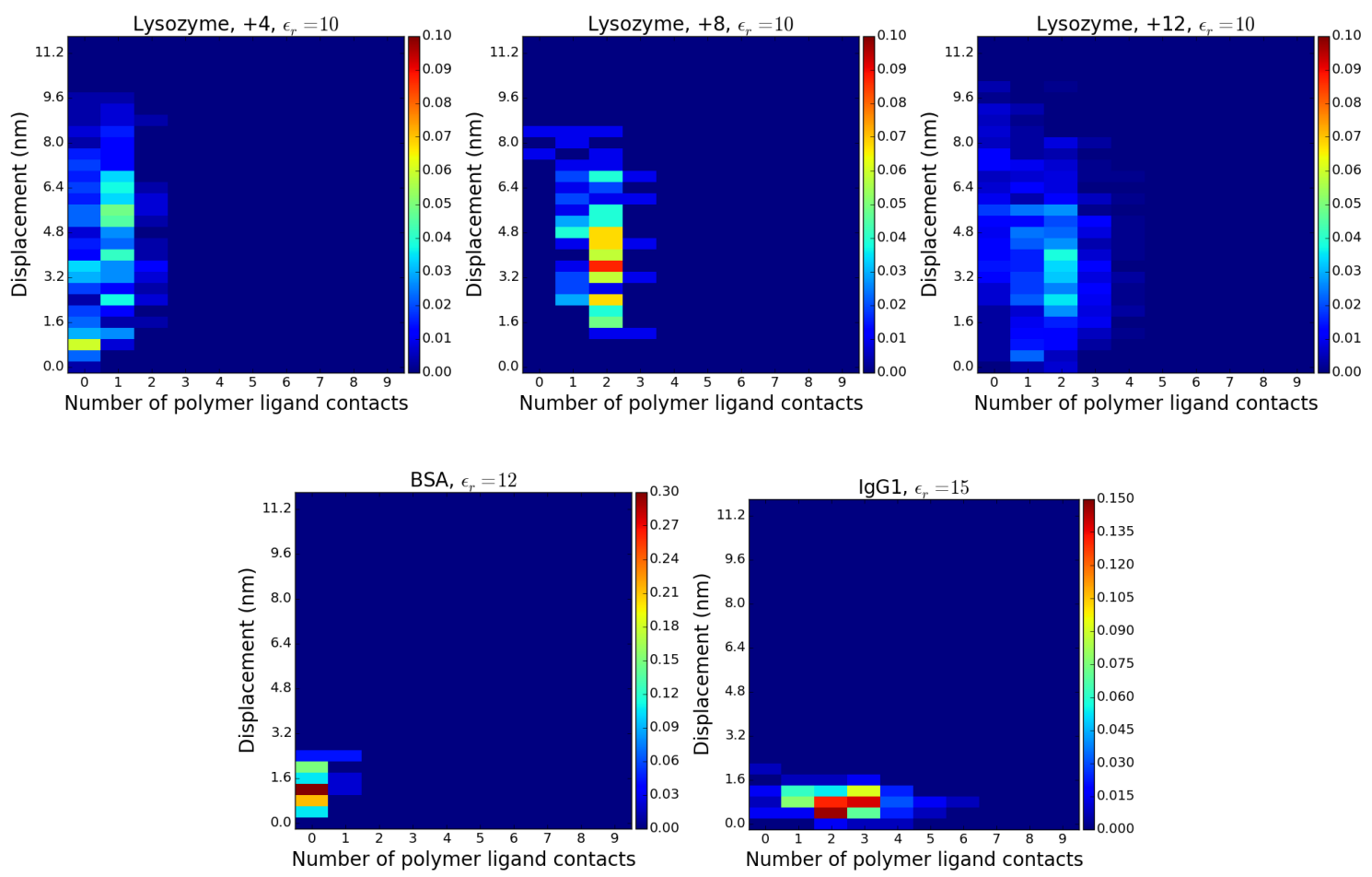

Figure 4.12: Histograms of protein displacement over a given time window versus the average number of polymer ligand contacts during that window. The upper plots show results for the lysozyme $+4,+8$, and +12 from left to right charge variants over a $60 \mathrm{~ns}$ window. The occurrence of large displacements when lysozyme is unadsorbed increases slightly with the charge of the protein. The lower plots show results for BSA and IgG1 over a $30 \mathrm{~ns}$ window on the left and right, respectively. These proteins exhibit similar magnitude displacements for different numbers of polymer-ligand contacts.

These plots illustrate that the most frequent levels of mobility and the associated number of polymer ligand contacts vary significantly for the different proteins. The width and locations of the high frequency zones on the $\mathrm{x}$ axis are consistent with the histograms shown in Fig. 4.11. The results for the lysozyme charge variants show that increasing the protein's charge leads to a greater oc- 
currence of large movements when the protein has 0 contacts with the polymer ligands, i.e. a higher density in the upper left portion of the plots. The distributions for BSA and IgG1 are clustered at moderate displacements with 0 polymer ligand contacts and 2 to 3 contacts, respectively.

According to previous studies, the degree to which the charged polymer grafts enhance transport depends on the competing effects of favorable protein-polymer ligand interactions and unfavorable steric hindrance by the polymer grafts on protein mobility. [61] Favorable protein-polymer ligand interactions lead to the so-called "chain-delivery" or "bucket-brigade" mechanism, which is believed to contribute significantly to the overall mass transfer flux. [22, 70] According to this theory, the diffusion of a protein molecule bound to a polymer graft is facilitated by the swinging of the flexible polymer towards other nearby polymers with unoccupied binding sites. This mechanism may be viewed a special case of the activated jump mechanism typically used to describe diffusion on a charged surface, as it involves the creation of a vacant binding site followed by the binding, or "jumping" of a molecule to this site. [70]

The chain delivery mechanism is believed to enhance transport more significantly as the density of polymer ligands and the charge of the protein increase, as both factors favor the formation of protein-ligand contacts, and thus the delivery of protein between neighboring polymers. [61] However, high polymer graft densities as well as large protein sizes lead to a more crowded pore space, and thus greater steric hindrance for protein diffusion. The factors of protein size, protein charge, and the density of charged polymer grafts all contribute to the overall transport rate, as seen in experimental measurements for $D_{e, a p p} / D_{0}$ for different proteins and polymer-grafted resins. [61]

Our modeling predictions for protein adsorption in the polymer-grafted pore 
are qualitatively consistent with these theories. For the +12 lysozyme variant under favorable binding conditions, the large enhancement in $D_{e, a p p} / D_{0}$ and the high number of polymer ligand contacts reflect a strong effect of the chain delivery mechanism and little steric hindrance due to the polymer grafts. Conversely, the very low $D_{e, a p p} / D_{0}$ and low number of polymer ligand contacts observed for BSA suggest that there is negligible enhancement due to the chain delivery effect, and significant steric hindrance. The large, highly-charged IgG1 should experience both enhanced diffusion due to the chain delivery effect by virtue of its high number of polymer ligand contacts, and steric hindrance to diffusion by the polymers due to its large size. The net result predicted by our simulations is an enhancement in $D_{e, a p p} / D_{0}$.

Finally, the MD simulations suggest that chain delivery of a given protein molecule may occur by either a "hand off", in which the protein always remains bound to a polymer ligand, or by desorption from a polymer ligand followed by a readsorption to a neighboring ligand. However, the heat maps shown in Fig. 4.12 suggest that these differing delivery mechanisms have limited effect on the overall protein mobility, at least for the proteins and charged polymers studied here. The frequency of a given displacement is generally independent of the average number of contacts, although as previously mentioned, highly charged, small proteins do exhibit more large movements when they are not in contact with any polymer ligands. The importance of this desorption/readsorption mechanism for chain delivery is expected to increase as the protein's size decreases, the protein's charge increases, and the space between neighboring polymer ligands increases. 


\subsubsection{Conclusions}

Multiscale modeling of different proteins in macroporous and polymer-grafted ion exchangers shows that adsorption behaviors vary significantly with the properties of the protein. MD simulations predict that partitioning into the polymerfilled pore space generally increases with respect to protein charge while surface adsorption is constant, leading to a net increase in the overall capacity. Higher protein charge also leads to enhanced adsorption kinetics in the polymer-grafted system relative to the macroporous, as predicted by multiscale simulation, due to the combined effects of increased pore space partitioning and only a modest reduction in diffusion in the pore space. The protein properties also affect the qualitative shape of the predicted adsorption front. Diffuse adsorption fronts and $D_{e, \text { app }} / D_{0}$ values of over 1 predicted for BSA and IgG1 in the macroporous systems suggest that the current molecular models may lead to overly high surface diffusion. Nevertheless, the predictions that the polymer grafts enhance the transport rate of IgG1 but not that of BSA, evident in $D_{e, a p p} / D_{0}$ values of approximately 6 and 1, respectively, agree qualitatively with previous experiments. Analysis of the number of contacts made between protein molecules and polymer ligands and protein mobility in the polymer-filled pore support existing hypotheses on how the chain delivery mechanism can enhance mass transfer diffusion in these systems. [61] 


\section{Chapter 5}

\section{Conclusions and Recommendations}

\subsection{Conclusions}

The multiscale modeling approach developed in this work has proven to be an effective tool for studying the relationship between the molecular properties of polymer-grafted IEC systems and macroscopic adsorption behaviors. Our simulations have provided insights and supported existing hypotheses on how adsorption occurs in these systems, which could help improve the design of IEC resins for future simulations. The following conclusions can be made from our work:

1. Enhanced transport in polymer-grafted IEC resins results from enhanced protein partitioning into pore space, and relatively fast diffusion of the protein in this region. These results support the previously-known empirical relationship $D_{e}=D^{\prime} q^{*} / C$ with a slightly more detailed model that distinguishes between the contributions of protein on the surface and in the pore space to the overall flux.

2. Protein partitioning into the pore space and the effective transport rate can 
be enhanced by various resin modifications that the increase polymer ligand content, such as increasing the polymer graft density or the charge content per polymer, at least for the model protein lysozyme.

3. At low protein loadings, the charged polymer grafts in certain IEC resins show a propensity to collapse onto the surface due to electrostatic attraction to surface-bound protein.

4. Protein partitioning into the pore space and the effective transport rate increase with the charge of the protein, which in some cases can compensate for steric exclusion effects of the polymers on large proteins, which would otherwise decrease the capacity and transport rate. The predicted effects of protein charge and size on adsorption performance in a polymer-grafted pore support the "chain delivery" mechanism for how transport is enhanced in these systems.

\subsection{Recommendations}

The ultimate goal of these modeling efforts is to provide insights into how adsorption and transport occur in polymer-grafted IEC resins, which could aid in the design of improved resins. It is difficult to predict based on our modeling results how a particular resin property should be designed to increase the binding capacity and/or uptake rate, due to both the approximate nature of our models and the difficult in synthesizing resin particles with precise attributes. Nevertheless, our results support previous theories stating that adsorption and transport are both enhanced by increased partitioning of protein into the entire pore space, due to favorable interactions with the charged polymer grafts which still allow 
for relatively high protein mobility. MD simulations suggest that this pore space partitioning depends on the competing effects of favorable protein-polymer ligand interactions and unfavorable steric exclusion of the protein by the polymer grafts. Thus, resin properties that maximize protein-polymer ligand interactions while not overly excluding the protein from the pore space or overly restraining protein mobility should be desirable for improving adsorption performance. Depending on the nature of the protein to be captured, the graft density, charge content per polymer, and flexibility of the polymer might each be manipulated to obtain the desirable molecular behaviors mentioned above.

Additionally, we have several recommendations for how this work could be extended in order to improve our understanding of protein adsorption in polymergrafted IEC systems, as well as the opportunities and limitations of multiscale modeling of these systems.

1. A more detailed analysis of the mechanism by which protein molecules interact with the charged polymers could provide valuable insights into the optimal chemical and physical design of polymer-grafted resins. For instance, diffusion in the pore space could be modeled in terms of the kinetics of protein desorption and readsorption from and to the charged polymers, and if applicable, on and off of the charged surface, though such events were rarely observed in our work. A detailed quantification of protein-polymer interactions could be correlated with predicted adsorption capacities and kinetics to better understand how molecular properties impact macroscopic observables.

2. Understanding the limitations of the current CG molecular models is necessary to determine under what scenarios modeling predictions are useful. 
Specifically, the issues encountered upon extending the model to larger proteins should be resolved, as large therapeutic proteins are of great commercial interest. The unphysical aggregation of BSA and the mAb in solution should be resolved in order to probe the effects of salt concentration on the adsorption of these proteins. Also, the unphysically high transport rates predicted for these proteins in the macroporous system should be understood and resolved, to ensure that the predicted contributions of the surface and the polymers to the overall flux are consistent with experiments. It is likely that the pore geometry contributes to the overly high transport rates observed here.

3. Efforts to validate the CG models with atomistic MD simulations should be continued. While the GLYCAM force field used to simulated dextran appears to gives overly-collapsed equilibrium structures, other force fields may yield more realistic polymer structures suitable for simulating proteinpolymer interactions in fine detail and validating the CG models. Atomistic simulations could also provide valuable insight into the role of water and counter-ion displacement in protein adsorption and diffusion within the polymers.

4. Finally, the multiscale modeling approach should be extended to additional IEC systems, when appropriate, to gain further insights into the relationships between the charge and size of the protein, the properties of the polymer-grafted resin, operating conditions, and adsorption performance. For example, the hypothesis that adsorption capacities generally increase with charge could be further tested by simulating mAbs with similar sizes but varying net charges. 


\section{Appendix A}

\section{Molecular model details}

\section{A.1 Protein model}

Each protein molecule in our MD simulations is represented by a coarse-grained (CG) model consisting of an elastic network of CG beads, with each bead representing the excluded volume, mass, and net charge of $n_{C G}$ or $n_{C G}+1$ contiguous residues. The studies described in Chapters 1 and 2 use lysozyme as the model protein, which has a molecular mass of $14.5 \mathrm{kDa}, 129$ amino acid residues, a $\mathrm{pI}$ of 11, and a net charge of +8 at $\mathrm{pH}$ 7. [71] The initial coordinates of each bead are found by averaging the positions of the $\alpha$-carbon atoms of contiguous residues, as obtained from the lysozyme crystal structure (PDB code 1AKI). [63] The mass and charge of each bead are found by summing these quantities for the same residues.

The appropriate level of coarse-graining for the protein model is defined as that which gives a charge distribution consistent with that of a fully atomistic protein model. CG models with different integer numbers of residues per bead, $n_{C G}$, varying from 1 to 9 , were considered. When the total number of residues is 
not evenly divisible by $n_{C G}$, the remaining residues are added to beads that have a neutral net charge, with a maximum of 1 additional residue per bead, in order to preserve the correct charge distribution in more highly-charged regions. For each model with a different level of coarse-graining, the electrostatic potential $V_{C G}$ is evaluated at approximately 200 points on a rectangular lattice surrounding the protein molecule, with each point separated from its nearest neighbors by $0.8 \mathrm{~nm}$. The potential at each point is compared to the potential of the fully atomistic model, $V_{\text {atom }}$, evaluated at the same location. Deviations from linearity between $V_{C G}$ and $V_{\text {atom }}$ for all points reflect a loss of information in the local charge density due to coarse-graining. For lysozyme, models with $n_{C G}=6$ or higher exhibit a significant increase in the error of the linear fit to $V_{C G}$ vs. $V_{\text {atom }}$. Therefore, the model with $n_{C G}=5$ (25 total beads) is used in our production simulations. It provides a good representation of the true charge distribution, with an $R^{2}$ value for the linear fit to $V_{C G}$ vs. $V_{\text {atom }}$ of over 0.99 , and provides improved computational efficiency versus more detailed models. Figs. S2 and S3 show $V_{C G}$ versus $V_{\text {atom }}$ for three representative coarse-grained models, and the standard error of the least square fit to $V_{C G}$ vs. $V_{\text {atom }}$ for all models, respectively. 


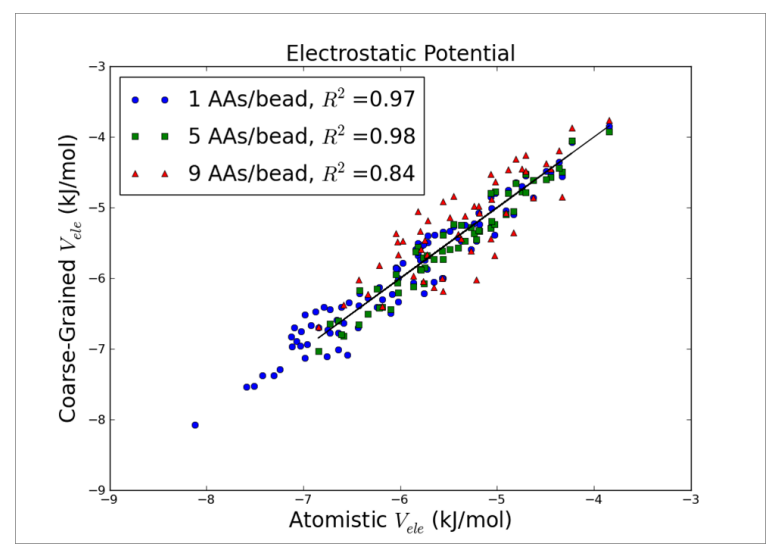

Figure A.1: Electrostatic potential at various locations surrounding CG models for lysozyme versus electrostatic potential at same locations surrounding an atomistic model.

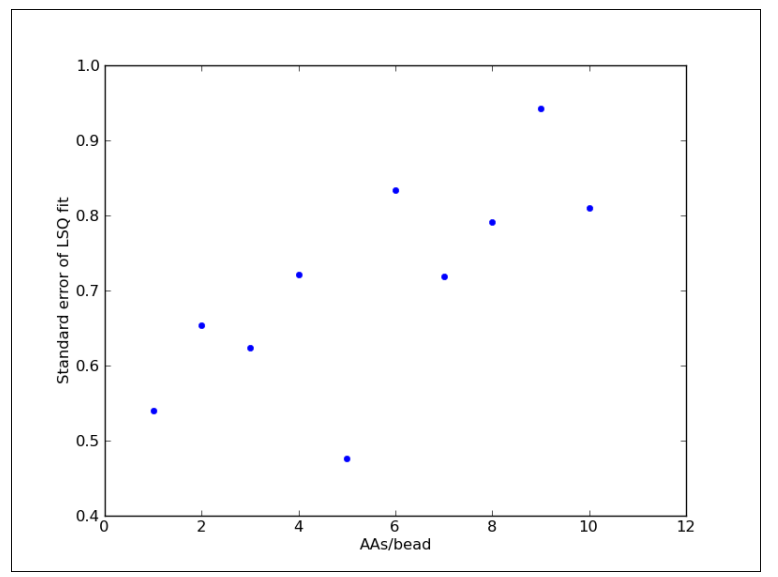

Figure A.2: Standard errors of least squares fits to $V_{C G}$ vs. $V_{\text {atom }}$ for all tested CG lysozyme models.

The protein structure is maintained using an elastic network model to define harmonic bonds between all coarse-grained particle pairs whose separation distance, $r_{i j}$, is $1.5 \mathrm{~nm}$ or less in the initial structure. The excluded volume of each bead is modeled using a purely-repulsive Lennard-Jones (LJ) potential of the form 
$V_{L J}=C_{12} / r_{i j}^{12}$, where $C_{12}=4 \epsilon_{i} \sigma_{i}^{12}$ and $\sigma_{i}$ and $\epsilon_{i}$ are the LJ radius and energy well depth, respectively, of a coarse-grained bead $i$. Although for a repulsive potential only the product $4 \epsilon_{i} \sigma_{i}^{12}$ is defined, we determine the two terms $\sigma$ and $\epsilon$ terms individually as described below.

$\epsilon_{i}$ is simply the sum of the $\epsilon$ values for all non-hydrogen atoms in the residues that comprise the bead, obtained from the OPLS-AA force field. $\sigma_{i}$ is dependent on the volumes of the residues, $V_{\text {res }}$, and the void fraction of the bead, $F_{i}$, due to empty space between the residues. We initially determined $\sigma_{i}$ for the $n_{C G}=1$ model (in which each CG bead represents a residue) based on the LJ radius $\sigma_{n}$ of each non-hydrogen atom in the residue, again based on the OPLS-AA parameters. Approximating each CG bead's total volume as $V_{i}=1 / F_{i} \sum_{n} V_{n}$, we estimated the $\mathrm{LJ}$ radius as $\sigma_{i}=\left(1 / F_{i} \sum_{n} \sigma_{n}^{3}\right)^{1 / 3}$. For this model (which is not actually used in simulations), an arbitrary void fraction of $F_{i}=0.5$ is applied to each bead. The same expression for $\sigma_{i}$ is then used to determine the radius of each bead in the $n_{C G}=5$ model where $n$ now represents the different radii of the $n_{C G}=1$ beads. Again a uniform $F_{i}$ is applied to each bead.

A series of MD simulations are used to determine appropriate values for the parameters $k_{b}$ and $F_{i}$ of the $n_{C G}=5$ model, which led to correct flexibility and size of the protein molecule. A 2 ns MD simulation of the fully atomistic model of lysozyme in SPC/E water is used as the standard for this parameterization. The protein's trajectory from the atomistic simulation was first mapped onto the coordinate system of the CG model, and the root mean square deviation (RMSD) from the initial structure and the radius of gyration $\left(R_{g}\right)$ were calculated as measures of the protein's flexibility and size, respectively. The CG model was then simulated using different $k_{b}$ and $F_{i}$ values and the same implicit solvent approach described previously. These simulations showed that the parameters $F_{i}=0.25$ and $k_{b}=$ 
$1500 \mathrm{~kJ} / \mathrm{mol}$ provided a good tradeoff between approximately the appropriate size and flexibility of the protein molecule. The $\sigma_{i}$ values corresponding to this $F_{i}$ range from 0.70 to $0.83 \mathrm{~nm}$. This model yields a $R_{g}$ of $1.36 \mathrm{~nm}$ and a RMSD of $0.05 \mathrm{~nm}$, while the atomistic model exhibits a $R_{g}$ of $1.37 \mathrm{~nm}$ and a RMSD of 0.08 $\mathrm{nm}$ (with errors of $1 \%$ or less for all cases). While the chosen coarse-grain model is somewhat less flexible that the atomistic, this property is not expected to impact the adsorption and diffusion results significantly compared to electrostatic interactions.

\section{A.2 Polymer graft model}

The dextran polymer model is designed to exhibit a persistence length consistent with physical dextran and an excluded volume consistent with that of the lysozyme model. These criteria are satisfied by the 1 bead/monomer model, though more and possibly less detailed models could also meet the criteria. The excluded volume of each monomer is described by a purely repulsive LJ potential, and protein molecules are attracted to the polymers solely through electrostatic interaction with the charged monomers. This model assumes that dispersion forces between the protein and the neutral polymer are canceled out by dispersion between proteins and the fictitious solvent. To ensure that the relative sizes of the polymer and protein are consistent, $\sigma_{\text {dextran }}$ is calculated from the average radius of the protein's CG beads, $\sigma_{\text {prot,avg }}$, assuming that the mass $m$ of each CG particle is proportional to its volume, such that $\sigma_{\text {dextran }}=\left(m_{\text {dextran }} / m_{\text {prot }, \text { avg }}\right)^{1 / 3}\left(\sigma_{\text {prot,avg }}\right)$. For the $n_{C G}=5$ protein model this leads to $\sigma_{\text {dextran }}=0.51 \mathrm{~nm}$. $\epsilon_{\text {dextran }}$ is estimated as the sum of the $\epsilon$ values of all non-hydrogen atoms in a dextran monomer.

Bonds between adjacent monomer are described by the Finitely Extensible 
Nonlinear Elastic (FENE) potential, $V_{\text {bond }}=\frac{1}{2} k_{F}\left(r_{i j}-b_{F}\right)^{2}$, where $b_{F}$ is the maximum allowed separation between a bonded pair and $k_{F}$ is the spring constant. We define $b_{F}=1.5 \sigma_{\text {dextran }}$ and $k_{F}=25 k_{B} T / \sigma_{\text {dextran }}$ (with $k_{B}$ the Boltzmann constant and $T=300 \mathrm{~K}$ ), as these conventions have been found previously to provide an appropriate amount of polymer stretching. [72] To assess the structural flexibility of the model dextran, we calculated the chain's persistence length, $L_{p}$, according to $\left\langle\cos \theta_{i j}\right\rangle=\exp \left(-(j-i) l / L_{p}\right)$, where $\theta_{i j}$ is the angle between consecutive bonds $i$ and $j$, and $l$ is the distance between two monomers. A 250-membered neutral chain simulated in vacuum with Langevin dynamics exhibited $L_{p}=0.61 \pm 0.04$ $\mathrm{nm}$, which is within a range of experimental values reported for physical dextran of $0.4 \mathrm{~nm}$ [73] to $0.65 \mathrm{~nm}$. [74] Each chain is "grafted" to the surface by fixing the position of its first monomer at a location near the surface. The fixed monomers are distributed laterally in a hexagonal arrangement such that each is equidistant to its nearest neighbors, and each is placed at the height of the would-be nearest surface ligand, which is replaced by the monomer.

The simulation box includes a void volume adjacent to the pore that is accessible to the protein but not to the charged polymers, to facilitate measurement of the protein concentration in bulk solution in equilibrium with the adsorbent pore. The polymer grafts are confined to the pore volume by two walls of purely repulsive particles whose positions are fixed at $y=0$ and $y=y_{\text {pore }}$, the boundaries between the pore and the void volumes. These particles interact only with the polymers, allowing protein molecules to access both the pore and the void. The particles are arranged in a hexagonal close packing arrangement with a separation of $1 \mathrm{~nm}$ from their nearest neighbors, and have the same $C_{12}$ parameter used for the agarose walls. Simulations of systems with different pore lengths $\left(y_{\text {pore }}=34.8 \mathrm{~nm}\right.$ and $\left.69.6 \mathrm{~nm}\right)$ exhibit time-averaged protein concentrations within 
the polymer phase that are statistically indistinguishable, indicating that any effects of the repulsive walls on the polymer phase structure does not affect protein affinity for this phase. For all systems, $x_{b o x}=x_{p o r e}$ and $y_{b o x}=3 y_{p o r e}$, such that the length of the void region is twice as long as the pore length. Periodic boundary conditions are used in the $x$ and $y$ dimensions.

The number of chains per surface and lateral dimensions of the pore, $x_{\text {pore }}$ and $y_{\text {pore }}$, are varied depending on the graft density. The $\sigma=\sigma_{e}$ system (used for the studies in Chapters 1 and 3) has $x_{\text {pore }}=40.2 \mathrm{~nm}, y_{\text {pore }}=34.8 \mathrm{~nm}$, and $n_{\text {chains }}=4$. For the adsorbent property studies described in Chapter2, the $\sigma=2 \sigma_{e}$ system has $x_{\text {pore }}=28.4 \mathrm{~nm}, y_{\text {pore }}=49.2 \mathrm{~nm}$, and $n_{\text {chains }}=8$, and the $\sigma=3 \sigma_{e}$ system has $x_{\text {pore }}=23.2 \mathrm{~nm}, y_{\text {pore }}=40.2 \mathrm{~nm}$, and $n_{\text {chains }}=8$. All systems have a box height of $z_{b o x}=28 \mathrm{~nm}$ and pore diameter (i.e. the shortest distance between ligands on the opposite surfaces) of $z_{\text {pore }}=21 \mathrm{~nm}$.

\section{A.2.1 Solvent Environment}

The simulations are performed in the NVT ensemble and evolve over time according to the Langevin equation of motion:

$$
m_{i} \frac{d^{2} r_{i}}{d t^{2}}=-m_{i} \xi_{i} \frac{d r_{i}}{d t}+F_{i}\left(r_{i}\right)+\hat{R}_{i}
$$

where $r_{i}, m_{i}$, and $F_{i}$ are the positions, masses, and forces acting on particle $i$, respectively. $\xi_{i}$ is a friction constant inversely proportional to the coupling strength $\tau_{T}$, and is applied uniformly to all particles in our simulations. $\hat{R}_{i}$ is a stochastic noise term with mean zero and $\left\langle\hat{R}_{i}(t) \hat{R}_{j}(t+\Delta t)\right\rangle=2 m_{i} \xi_{i} k_{B} T \delta(\Delta t) \delta_{i j}$ where $\Delta t$ the time offset and $i$ and $j$ are the indices of any two particles.

An integration time step of 6 fs is used, which is the largest for which energy 
is well-conserved in these systems. The coupling strength $\tau_{T}$ of the Langevin equation controls the magnitude of the friction and stochastic noise terms applied to each particle, which we utilize to approximate the dynamics of a solvated system without using explicit solvent molecules. [33] In simulations used to measure protein diffusion, $\tau_{T}$ is tuned to $0.7 \mathrm{ps}$, which leads to an average diffusivity of CG lysozyme molecules in vacuum that is consistent with lysozyme's experimental free solution diffusivity of $D_{0}=1.2 \times 10^{-6} \mathrm{~cm}^{2} / \mathrm{s}$. [68] In simulations used to measure protein partitioning, $\tau_{T}$ is set to $100 \mathrm{ps}$, which provides less dampening of the dynamics and thus accelerates the adsorption of protein onto the surface, without affecting the thermodynamics governing the equilibrium adsorption behavior. The Langevin equation also serves as a thermostat, maintaining the average system temperature at $T_{r e f}=300 \mathrm{~K}$.

Electrostatic interactions are represented by the "reaction field" potential [75], $V_{c r f}$, between all charged particle pairs separated by a distance $r_{i j}$ less than the cut-off $r_{c}$ :

$$
V_{c r f}=f \frac{q_{i} q_{j}}{\epsilon_{r}}\left[\frac{1}{r_{i j}}+k_{r f} r_{i j}^{2}-c_{r f}\right]
$$

where $k_{r f}=\left(1 / r_{c}^{3}\right)\left(\epsilon_{r f}-\epsilon_{r}\right) /\left(2 \epsilon_{r f}+\epsilon_{r}\right)$ and $c_{r f}=\left(1 / r_{c}\right)\left(3 \epsilon_{r f}\right) /\left(2 \epsilon_{r f}+\epsilon_{r}\right)$. The inner dielectric constant $\epsilon_{r}$ governs the electrostatic interaction between two particles separated by $r_{i j}<r_{c}$, while the outer dielectric $\epsilon_{r f}$ represents a homogeneous charged environment at distances beyond $r_{c} . \epsilon_{r f}=80$ is used to represent the screening of charges by water. In our simulations, the inner dielectric $\epsilon_{r}$ is tuned to represent qualitatively the screening of electrostatics by the solvent and $\mathrm{co}^{-}$ and counter-ions. While this approach obviously cannot capture competition between ions and protein molecules for binding sites or polarization caused by 
reorientation of water dipoles, it does provide a simplified way to control the relative ionic strength. A large $\epsilon_{r}$ significantly screens charged interactions and reduces protein affinity for the adsorbent similarly to a high salt concentration, while a lower $\epsilon_{r}$ is representative of more favorable binding conditions at low salt concentration.

The cutoff for the reaction field potential, $r_{c}=2.58 \mathrm{~nm}$, as well as the cutoffs for the neighbor list, $r_{l i s t}=2.53 \mathrm{~nm}$, and for Lennard-Jones interactions, $r_{v d w}=$ $2.47 \mathrm{~nm}$, are approximately 5 times the average radius of the CG beads of the lysozyme protein model, $\sigma_{\text {prot,avg }}$ (the choice of $\sigma_{\text {prot,avg }}$ is discussed below). Trial MD simulations of the macroporous system with different $r_{c}$ values (with $r_{\text {list }}$ and $r_{v d w}$ scaled proportionally, $\epsilon_{r}=10$, and $N=210$ protein molecules) show that the radial distribution of protein molecules relative to the charged particles on the surface becomes invariant to the cutoff at $r_{c} \approx 5 \sigma_{\text {prot,avg }}$, as seen in Fig. A.3. 


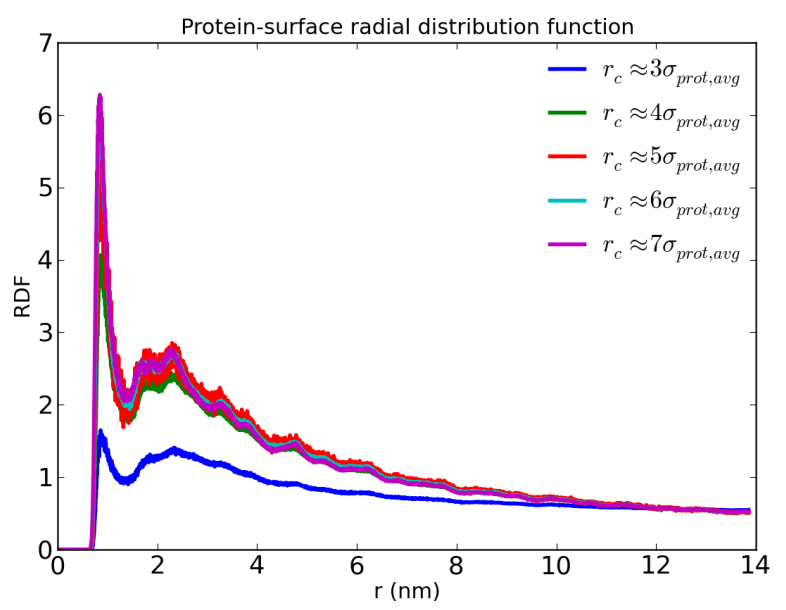

Figure A.3: The equilibrium surface adsorption behavior of lysozyme becomes invariant to the cutoff radius for the reaction field electrostatic potential, $r_{c}$, at sufficiently long $r_{c}$, as shown in radial distribution functions of protein molecules relative to the charged particles on the surface. 


\section{Appendix B}

\section{Model parameterization simulations}

Certain details of the macroporous and polymer-grafted molecular models are not directly constrained by experimental data. These include the inner dielectric constant, $\epsilon_{r}$, used to represent favorable binding conditions, the density and spatial distribution of ligands on the surface, and the charge content per grafted dextran in the polymer-grafted system. To determine parameter values for these details that are consistent with experimental trends for the effective transport rate, a series of multi-scale simulations were performed using different model parameters. In parameter sets $\mathrm{A}-\mathrm{C}$, the distribution of surface ligands was defined by the 2D sine wave amplitude $A_{\text {surf }}=2 / 3 d_{\text {prot }}$ and period $T_{\text {surf }}=d_{\text {prot }}$, where $d_{\text {prot }}=3 \mathrm{~nm}$, the approximate diameter of the lysozyme protein molecule. The surface of parameter set D has $A_{\text {surf }}=1 / 3 d_{\text {prot }}$ and $T_{\text {surf }}=d_{\text {prot }}$, i.e. lower peaks and troughs.

Parameter set D was used for for production simulations, as these model parameters lead to transport and adsorption behaviors most consistent with the physical systems. Specifically, the effective transport rate in the macroporous system approaches the result expected for experimental macroporous systems, 
i.e. $D_{e} / D_{0} \approx 1.0$, indicating an ordinary pore diffusion mass transfer mechanism. The effective transport rate in the polymer-grafted system approaches the experimental result for lysozyme in a dextran-grafted adsorbent of $D_{e} / D_{0}=5.0$. [9] Finally, these parameters lead to a homogeneous monolayer of surface-adsorbed protein, as is expected for these materials. Parameter set $\mathrm{C}$ exhibits comparable transport rates, however, the parameters for the surface ligand distribution lead to two different types of adsorption sites. The different equilibrium surface adsorption behaviors observed with parameter sets D and C are shown in the solid and dashed lines, respectively, of Fig. S5, which plots the distribution of protein molecules relative to the charged surface.

\begin{tabular}{|c|ccc|cc|}
\hline & \multicolumn{3}{|c|}{ Model details } & \multicolumn{2}{c|}{ Adsorption behaviors } \\
\hline Parameter & $\epsilon_{r}$ & $\begin{array}{c}n_{S L} / S \\
\left(\text { ligands } / 100 \mathrm{~nm}^{2}\right)\end{array}$ & $n_{S L} / n_{\text {poly }}$ & $\begin{array}{c}D_{e} / D_{0} \\
\text { Macro }\end{array}$ & $\begin{array}{c}D_{e} / D_{0} \\
\text { Poly-grafted }\end{array}$ \\
\hline $\mathrm{A}$ & 8 & 29 & 0.05 & $1.9(0.1)$ & $16.7(0.1)$ \\
$\mathrm{B}$ & 10 & 29 & 0.05 & $2.0(0.1)$ & $9.1(0.6)$ \\
$\mathrm{C}$ & 10 & 49 & 0.05 & $1.7(0.3)$ & $5.3(0.8)$ \\
$\mathrm{D}$ & 10 & 49 & 0.05 & $1.3(0.1)$ & $6.1(0.6)$ \\
\hline
\end{tabular}

Table B.1: Summary of relevant lysozyme adsorption behaviors observed experimentally and predicted from multi-scale simulation using models with various parameter values for the charge content of the adsorbent and the inner dielectric constant, $\epsilon_{r}$.

The surface model also significantly affects the diffusional mobility of surfacebound protein. Table S2 gives $D_{s}$ values measured from MD simulations conducted with different values of $A_{\text {surf }}$ and $T_{\text {surf }}$ and $n_{S L} / S$ of 29 ligands $/ 100 \mathrm{~nm}^{2}$. All systems were simulated with a protein loading of $N=170$ which led to a nearly saturated surface and $\epsilon_{r}=12$. The predicted $D_{s}$ values range from $1 \times 10^{-8}$ to $1 \times 10^{-7} \mathrm{~cm}^{2} / \mathrm{s}$. Models with a sine wave period of $T<d_{\text {prot }}$ generally lead to faster diffusion, as protein molecules cannot access the troughs of the surface. Experimental measurements for $D_{s}$ vary depending on the type of protein and 


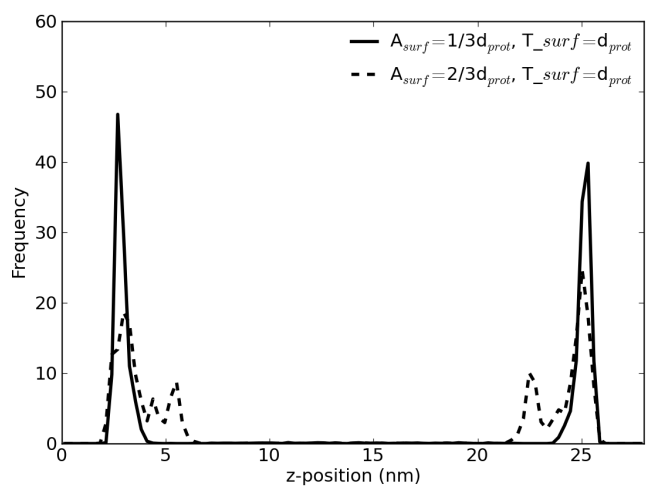

Figure B.1: Number densities of lysozyme protein molecules with respect to $z$ position in a macroporous adsorbent under saturation conditions, for two different distribution of ligands on the surface. In both cases $\epsilon_{r}=10$. A surface ligand distribution that follows a 2-D sine wave with amplitude $A_{\text {surf }}=1 / 3 d_{\text {prot }}$ and period $T_{\text {surf }}=d_{\text {prot }}$ exhibits a single type of adsorption site (solid curve). A surface with an amplitude of $A_{\text {surf }}=2 / 3 d_{\text {prot }}$ and the same period exhibits two different sites (dashed curve), at which protein molecules interact with either multiple ligands or a single ligand.

adsorbent, but are generally on the order of $1 \times 10^{-8} \mathrm{~cm}^{2} / \mathrm{s}$. [5] Surface diffusion is expected to have a negligible contribution to overall mass transfer in the ionexchangers considered here, [20] and therefore the parameters which provided the lowest $D_{s}$ values, $A=2 / 3 d_{\text {prot }}$ and $T=d_{\text {prot }}$, are used for production simulations. We also tested a smooth surface model in which all surface ligands had a uniform height, which led to surface diffusion of $D_{s}=1.7 \times 10^{-7} \mathrm{~cm}^{2} /$ sfor $\epsilon_{r}=12$, indicating that some surface inhomogeneity is necessary to obtain the expect diffusion behavior. 


\begin{tabular}{|lllll|}
\hline & $T=1 / 3 d_{\text {prot }}$ & $T=2 / 3 d_{\text {prot }}$ & $T=d_{\text {prot }}$ & $T=4 / 3 d_{\text {prot }}$ \\
\hline$A=1 / 3 d_{\text {prot }}$ & $7.2(0.5)$ & $5.3(0.5)$ & $3.9(0.4)$ & $6.9(0.5)$ \\
$A=2 / 3 d_{\text {prot }}$ & $11.0(0.6)$ & $5.6(0.4)$ & $3.0(0.3)$ & $3.2(0.3)$ \\
$A=d_{\text {prot }}$ & $20.7(1.1)$ & $8.3(0.6)$ & $6.9(0.5)$ & $5.9(0.5)$ \\
$A=4 / 3 d_{\text {prot }}$ & $21.5(1.2)$ & $8.5(0.6)$ & $8.5(0.6)$ & $6.5(0.5)$ \\
\hline
\end{tabular}

Table B.2: Average diffusivity of surface-adsorbed lysozyme protein, $D_{s}$, as a function of the amplitude $A$ and period $T$ of the $2 \mathrm{D}$ sine wave defining the heights of SP groups on surface. All values have units of $1 \times 10^{-8} \mathrm{~cm}^{2} / \mathrm{s}$, and averages and associated uncertainties are calculated from an ensemble of surfaceadsorbed protein molecules. The surface model with $A=2 / 3 d_{\text {prot }}$ and $T=d_{\text {prot }}$, which gives at surface diffusion as slower or slower than any other model, is used for production simulations. 


\section{Bibliography}

[1] Rickwood, S. and Kleinrock, M. and Núñez-Gaviria, M. The global use of medicines: Outlook through 2017. Technical report, IMS Institute for Healthcare Informatics, 2013.

[2] D. L. Hacker, M. De Jesus, and F. M. Wurm. 25 years of recombinant proteins from reactor-grown cells - Where do we go from here? Biotechnol. Adv., 27 (6):1023-1027, 2009.

[3] A. A. Shukla, B. Hubbard, T. Tressel, S. Guhan, and D. Low. Downstream processing of monoclonal antibodies - Application of platform approaches. J. Chromatogr. B, 848(1):28-39, 2007.

[4] U. Gottschalk. Process Scale Purification of Antibodies. Wiley, 2009.

[5] G. Carta and A. Jungbauer. Protein Chromatography: Process Development and Scale-Up. Wiley-VCH, 2010.

[6] A. Jungbauer. Chromatographic media for bioseparation. J. Chromatogr. A, 1065(1):3-12, 2005.

[7] J. Thömmes. Investigations on protein adsorption to agarose-dextran composite media. Biotechnol. Bioeng., 62(3):358-362, 1999. 
[8] W. Müller. New ion exchangers for the chromatography of biopolymers. J. Chromatogr. A, 510(0):133-140, 1990.

[9] M. C. Stone and G. Carta. Protein adsorption and transport in agarose and dextran-grafted agarose media for ion exchange chromatography. J. Chromatogr. A, 1146(2):202-215, 2007.

[10] B. D. Bowes, H. Koku, K. J. Czymmek, and A. M. Lenhoff. Protein adsorption and transport in dextran-modified ion-exchange media. I: Adsorption. J. Chromatogr. A, 1216(45):7774-7784, 2009.

[11] E. J. Suda, K. E. Thomas, T. M. Pabst, P. Mensah, N. Ramasubramanyan, M. E. Gustafson, and A. K. Hunter. Comparison of agarose and dextran-grafted agarose strong ion exchangers for the separation of protein aggregates. $J$. Chromatogr. A, 1216(27):5256-5264, 2009.

[12] T. Kawai, K. Saito, and W. Lee. Protein binding to polymer brush, based on ion-exchange, hydrophobic, and affinity interactions. J. Chromatogr. B, 790 (1-2):131-142, 2003.

[13] E. Boschetti. Advanced sorbents for preparative protein separation purposes. J. Chromatogr. A, 658(2):207-236, 1994.

[14] C. Chang and A. M. Lenhoff. Comparison of protein adsorption isotherms and uptake rates in preparative cation-exchange materials. J. Chromatogr. A, 827(2):281-293, 1998.

[15] A. M. Lenhoff. Protein adsorption and transport in polymer-functionalized ion-exchangers. J. Chromatogr. A, 1218(49):8748-8759, 2011. 
[16] I. N. Savina, I. Y. Galaev, and B. Mattiasson. Ion-exchange macroporous hydrophilic gel monolith with grafted polymer brushes. J. Mol. Recognit., 19 (4):313-321, 2006.

[17] S. Tsuneda, K. Saito, S. Furusaki, and T. Sugo. High-throughput processing of proteins using a porous and tentacle anion-exchange membrane. $J$. Chromatogr. A, 689(2):211-218, 1995.

[18] N. D. Marlin and N. W. Smith. Separation of biomolecules by micro-highperformance anion-exchange chromatography using a tentacle-like stationary phase. Anal. Bioanal. Chem., 382(2):493-497, 2005.

[19] K. H. A. Lau, C. Ren, S. H. Park, I. Szleifer, and P. B. Messersmith. An experimental-theoretical analysis of protein adsorption on peptidomimetic polymer brushes. Langmuir, 28(4):2288-2298, 2012.

[20] M. C. Stone, Y. Tao, and G. Carta. Protein adsorption and transport in agarose and dextran-grafted agarose media for ion exchange chromatography: Effect of ionic strength and protein characteristics. J. Chromatogr. A, 1216(20):44654474, 2009.

[21] B. D. Bowes and A. M. Lenhoff. Protein adsorption and transport in dextranmodified ion-exchange media. II. Intraparticle uptake and column breakthrough. J. Chromatogr. A, 1218(29):4698-4708, 2011.

[22] Y. Tao, E. X. Pérez-Almodóvar, G. Carta, G. Ferreira, and D. Robbins. Adsorption kinetics of deamidated antibody variants on macroporous and dextrangrafted cation exchangers. III. Microscopic studies. J. Chromatogr. A, 1218(44): 8027-8035, 2011. 
[23] B. D. Bowes and A. M. Lenhoff. Protein adsorption and transport in dextranmodified ion-exchange media. III. Effects of resin charge density and dextran content on adsorption and intraparticle uptake. J. Chromatogr. A, 1218(40): 7180-7188, 2011.

[24] L. L. Yu, S. P. Tao, X. Y. Dong, and Y. Sun. Protein adsorption to poly(ethylenimine)-modified Sepharose FF: I. A critical ionic capacity for drastically enhanced capacity and uptake kinetics. J. Chromatogr. A, 1305(0): 76-84, 2013.

[25] J. R. Thompson, A. J. Heron, Y. Santoso, and M. I. Wallace. Enhanced stability and fluidity in droplet on hydrogel bilayers for measuring membrane protein diffusion. Nano Letters, 7(12):3875-3878, 2007.

[26] D. M. Owen, D. Williamson, C. Rentero, and K. Gaus. Quantitative microscopy: Protein dynamics and membrane organisation. Traffic, 10(8):962971, 2009.

[27] R. A. Latour. Molecular simulation of protein-surface interactions: Benefits, problems, solutions, and future directions, (review). Biointerphases, 3(3):FC2 - FC12, 2008.

[28] L. Zhang and Y. Sun. Molecular simulation of adsorption and its implications to protein chromatography: A review. Biochem. Eng. J, 48(3):408-415, 2010.

[29] K. Kubiak-Ossowska and P. A. Mulheran. Mechanism of hen egg white lysozyme adsorption on a charged solid surface. Langmuir, 26(20):1595415965, 2010.

[30] E. Riccardi, J. C. Wang, and A. I. Liapis. A molecular dynamics study on the 
transport of a charged biomolecule in a polymeric adsorbent medium and its adsorption onto a charged ligand. J. Chem. Phys., 133(8), 2010.

[31] E. Riccardi, J. C. Wang, and A. I. Liapis. Molecular modeling of polymeric adsorbent media: The effects of counter-ions on ligand immobilization and pore structure. J. Sep. Sci, 35(22, SI):3073-3083, 2012.

[32] J. E. Basconi, G. Carta, and M. R. Shirts. Multiscale modeling of protein adsorption and transport in macroporous and polymer-grafted ion exchangers. AIChE J., 60(11):3888-3901, 2014.

[33] J. E. Basconi and M. R. Shirts. Effects of temperature control algorithms on transport properties and kinetics in molecular dynamics simulations. J. Chem. Theory Comput, 9(7):2887-2899, 2013.

[34] J. E. Basconi, G. Carta, and M. R. Shirts. Effects of polymer graft properties on protein adsorption and transport in ion exchange chromatography: A multiscale modeling study. Langmuir, 2015. doi: 10.1021/la504768g.

[35] M. Heitzig, G. Sin, P. Glarborg, and R. Gani. A computer-aided framework for regression and multi-scale-modelling needs in innovative productprocess engineering. Computer-Aided Chemical Engineering Series, 28:379-384, 2010.

[36] A. M. Lenhoff. Multiscale modeling of protein uptake patterns in chromatographic particles. Langmuir, 24(12):5991-5995, 2008.

[37] S. R. Dziennik, E. B. Belcher, G. A. Barker, M. J. DeBergalis, S. E. Fernandez, and A. M. Lenhoff. Nondiffusive mechanisms enhance protein uptake rates in ion exchange particles. Proc. Natl. Acad. Sci. U. S. A, 100(2):420-425, 2003. 
[38] A. R. Ubiera and G. Carta. Radiotracer measurements of protein mass transfer: Kinetics in ion exchange media. Biotechnol. J., 1(6):665-674, 2006.

[39] X. Zhang, J. C. Wang, K. M. Lacki, and A. I. Liapis. Molecular dynamics simulation studies of the transport and adsorption of a charged macromolecule onto a charged adsorbent solid surface immersed in an electrolytic solution. J. Colloid Interf. Sci, 277(2):483-498, 2004.

[40] F. Dismer and J. Hubbuch. 3D structure-based protein retention prediction for ion-exchange chromatography. J. Chromatogr. A, 1217(8):1343-1353, 2010.

[41] J. Liang, G. Fieg, F. J. Keil, and S. Jakobtorweihen. Adsorption of proteins onto ion-exchange chromatographic media: A molecular dynamics study. Ind. Eng. Chem. Res., 51(49):16049-16058, 2012.

[42] A. S. Freed, S. Garde, and S. M. Cramer. Molecular simulations of multimodal ligand-protein binding: Elucidation of binding sites and correlation with experiments. J. Phys. Chem. B, 115(45):13320-13327, 2011.

[43] H.-O. Johansson and J. M. Van Alstine. Modeling of protein interactions with surface-grafted charged polymers. correlations between statistical molecular modeling and a mean field approach. Langmuir, 22(21):8920-8930, 2006.

[44] E. Riccardi, J. C. Wang, and A. I. Liapis. The design by molecular dynamics modeling and simulations of porous polymer adsorbent media immobilized on the throughpore surfaces of polymeric monoliths. J. Chromatogr. Sci., 47 (6):459-466, 2009.

[45] K. Kubiak and P. Mulheran. Molecular dynamics simulations of hen egg white lysozyme adsorption at a charged solid surface. J. Phys. Chem. B, 113 (36):12189-12200, 2009. 
[46] W. Norde. Driving forces for protein adsorption at solid surfaces. Macromol. Symp., 103(1):5-18, 1996.

[47] K. D. Collins. Why continuum electrostatics theories cannot explain biological structure, polyelectrolytes or ionic strength effects in ion-protein interactions. Biophys. Chem., 167(Complete):43-59, 2012.

[48] Woods Group, Complex Carbohydrate Research Center, University of Georgia, Athens, GA. GLYCAM Web Kernel Description. http://www.glycam.com, 2014.

[49] K. N. Kirschner, A. B. Yongye, S. M. Tschampel, J. González-Outeiriño, C. R. Daniels, B. L. Foley, and R. J. Woods. GLYCAM06: A generalizable biomolecular force field. Carbohydrates. J. Comput. Chem., 29(4):622-655, 2008.

[50] M. Stone. Synthesis and characterization of functionalized dextran-agarose media for ion exchange chromatography. Master's thesis, University of Virginia, 2006.

[51] A. W. Schüttelkopf and D. M. F. van Aalten. PRODRG: a tool for highthroughput crystallography of protein-ligand complexes. Acta Crystallogr., Sect. D: Biol. Crystallogr., 60(8):1355-1363, Aug 2004.

[52] J. Wang, R. M. Wolf, J. W. Caldwell, P. A. Kollman, and D. A. Case. Development and testing of a general amber force field. J. Comput. Chem., 25(9): 1157-1174, 2004.

[53] J. N. Canongia Lopes, A. A. H. Pádua, and K. Shimizu. Molecular force field for ionic liquids iv: Trialkylimidazolium and alkoxycarbonyl-imidazolium cations; alkylsulfonate and alkylsulfate anions. J. Phys. Chem. B, 112(16): 5039-5046, 2008. 
[54] M. A. Masuelli. Dextrans in aqueous solution. experimental review on intrinsic viscosity measurements and temperature effect. Journal of Polymer and Biopolymer Physics Chemistry, 1(1):13-21, 2013.

[55] B. Hess, C. Kutzner, D. van der Spoel, and E. Lindahl. GROMACS 4: Algorithms for Highly Efficient, Load-Balanced, and Scalable Molecular Simulation. J. Chem. Theory Comput., 4(3):435-447, 2008.

[56] S. Bielecki, J. Polak, and J. Tramper. Food Biotechnology (Progress in Biotechnology). Elsevier Science, 2000.

[57] Y. M. Kim, A. Kimura, and D. Kim. Novel quantitative method for the degree of branching in dextran. Food Sci. Biotechnol, 20:537-541, 2011.

[58] W. E. Schiesser. The Numerical Method of Lines: Integration of Partial Differential Equations. Elsevier Science, 1991.

[59] E. Riccardi, J. C. Wang, and A. I. Liapis. Rational surface design for molecular dynamics simulations of porous polymer adsorbent media. J. Phys. Chem. B, 112(25):7478-7488, 2008.

[60] E. X. Pérez-Almodóvar, Y. Tao, and G. Carta. Protein adsorption and transport in cation exchangers with a rigid backbone matrix with and without polymeric surface extenders. Biotechnol. Prog., 27(5):1264-1272, 2011.

[61] Y. Hong, N. Liu, W. Wei, L. L. Yu, G. Ma, and Y. Sun. Protein adsorption to poly(ethylenimine)-modified Sepharose FF: III. Comparison between different proteins. J. Chromatogr. A, 1342(0):30-36, 2014.

[62] J. Guo, S. Zhang, and G. Carta. Unfolding and aggregation of a glycosylated monoclonal antibody on a cation exchange column. part i. chromatographic 
elution and batch adsorption behavior. J. Chromatogr. A, 1356(0):117-128, 2014.

[63] P. J. Artymiuk, C. C. F. Blake, D. W. Rice, and K. S. Wilson. The structures of the monoclinic and orthorhombic forms of hen egg-white lysozyme at 6 A resolution. Acta Crystallogr., Sect. B: Struct. Crystallogr. Cryst. Chem, 38(3): 778-783, 1982.

[64] K. A. Majorek, P. J. Porebski, A. Dayal, M. D. Zimmerman, K. Jablonska, A. J. Stewart, M. Chruszcz, and W. Minor. Structural and immunologic characterization of bovine, horse, and rabbit serum albumins. Mol. Immunol., 52(3-4):174-182, 2012.

[65] E. O. Saphire, P. W. H. I. Parren, R. Pantophlet, M. B. Zwick, G. M. Morris, P. M. Rudd, R. A. Dwek, R. L. Stanfield, D. R. Burton, and I. A. Wilson. Crystal Structure of a Neutralizing Human IgG Against HIV-1: A Template for Vaccine Design. Science, 293(5532):1155-1159, 2001.

[66] A. Šali and T. L. Blundell. Comparative protein modelling by satisfaction of spatial restraints. J. Mol. Biol., 234(3):779-815, 1993.

[67] R. Anandakrishnan, B. Aguilar, and A. V. Onufriev. H++ 3.0: automating $\mathrm{pK}$ prediction and the preparation of biomolecular structures for atomistic molecular modeling and simulations. Nucl. Acids Res., 40(W1):W537-W541, 2012.

[68] T. M. Tyn and T. W. Gusek. Prediction of diffusion coefficients of proteins. Biotechnol. Bioeng, 35:327-338, 1990.

[69] W.-D. Chen, X.-Y. Dong, and Y. Sun. Analysis of diffusion models for protein 
adsorption to porous anion-exchange adsorbent. J. Chromatogr. A, 962(1-2): 29-40, 2002.

[70] L. Yu, L. Zhang, and Y. Sun. Protein behavior at surfaces: Orientation, conformational transitions and transport. J. Chromatogr. A, 1382(0):118-134, 2015.

[71] D. E. Kuehner, J. Engmann, F. Fergg, M. Wernick, H. W. Blanch, and J. M. Prausnitz. Lysozyme net charge and ion binding in concentrated aqueous electrolyte solutions. J. Phys. Chem. B, 103(8):1368-1374, 1999.

[72] K. Binder. Monte Carlo and Molecular Dynamics Simulations in Polymer Science, pages 125-194. Oxford University Press, 1995.

[73] M. Rief, J. M. Fernandez, and H. E. Gaub. Elastically coupled two-level systems as a model for biopolymer extensibility. Phys. Rev. Lett, 81(21):47644767, 1998.

[74] K. Snoussi and B. Halle. Protein self-association induced by macromolecular crowding: A quantitative analysis by magnetic relaxation dispersion. Biophys. J, 88(4):2855-2866, 2005.

[75] J. A. Barker. Reaction field, screening, and long-range interactions in simulations of ionic and dipolar systems. Mol. Phys, 83(6):1057-1064, 1994. 